\title{
Recent developments in spectral stochastic methods for the numerical solution of stochastic partial differential equations
}

\author{
Anthony Nouy
}

\begin{abstract}
Uncertainty quantification appears today as a crucial point in numerous branches of science and engineering. In the last two decades, a growing interest has been devoted to a new family of methods, called spectral stochastic methods, for the propagation of uncertainties through physical models governed by stochastic partial differential equations. These approaches rely on a fruitful marriage of probability theory and approximation theory in functional analysis. This paper provide a review of some recent developments in computational stochastic methods, with a particular emphasis on spectral stochastic approaches. After a review of different choices for the functional representation of random variables, we provide an overview of various numerical methods for the computation of these functional representations: projection, collocation, Galerkin approaches... A detailed presentation of Galerkin-type spectral stochastic approaches and related computational issues is provided. Recent developments on model reduction techniques in the context of spectral stochastic methods are also discussed. The aim of these techniques is to circumvent several drawbacks of spectral stochastic approaches (computing time, memory requirements, intrusive character) and to allow their use for large scale applications. We particularly focus on model reduction techniques based on spectral decomposition techniques and their generalizations.
\end{abstract}

Keywords Computational Stochastic Methods - Stochastic partial differential equations - Spectral stochastic methods - Galerkin stochastic methods - Stochastic finite elements · Polynomial Chaos · Karhunen-Loève · Model Reduction · Generalized spectral decomposition · Separated Representations · Finite Sums Decomposition

This work is supported by the French National Research Agency (grant ANR-06-JCJC-0064) and by GdR MoMaS with partners ANDRA, BRGM, CEA, CNRS, EDF, IRSN.

A. Nouy

Research Institute in Civil Engineering and Mechanics (GeM), University of Nantes, Ecole Centrale Nantes, CNRS

2 rue de la Houssinière, 44322 Nantes, France

E-mail: anthony.nouy@univ-nantes.fr 


\section{Introduction}

The numerical simulation of physical models, supported by the availability of increasing computational resources, plays today a very significant role in design processes or in decision making which can have major consequences in economic or human terms. Uncertainty quantification and propagation in physical systems appear as a critical path for the improvement of the prediction of their response. Obtaining reliable predictions requires a perfect control of the conceptual model. Upstream, this model must be designed in order to accurately reproduce the observations or more specifically some observable quantities of interest. Physical models, which are generally quite well mastered within the deterministic framework, must then be supplemented by a relevant modeling of uncertainties. Downstream, a numerical model and ad hoc resolution techniques must be proposed in order to propagate uncertainties and to estimate their impact on the response of the conceptual model.

Two types of uncertainties are generally distinguished: the intrinsic (or aleatoric) uncertainties, associated with a natural variability of the considered physical phenomena, and the epistemic uncertainties, which result from a lack of knowledge. Of course, the latter ones are by nature reducible. However, due to the complexity of physical phenomena, to the lack of observations or even to the necessity of providing simplified mathematical models, addressing epistemic uncertainties appears also essential in order to improve the predictability of the model. The probabilistic framework is indisputably the most well established way to model uncertainties, both from theoretical and practical points of views. In this context, the modeling of uncertainties consists in defining a suitable probability space. For many physical problems, the conceptual model can then be expressed in terms of stochastic partial differential equations (SPDEs).

Many numerical methods have been proposed for the resolution of SPDEs. The mathematical framework for the analysis of the problem and the choice of a particular numerical solution strategy depend on the nature of uncertainties, on the quantities of interest to predict and on the expected accuracy of this prediction. A particular class of problems concerns the case of stochastic differential equations or stochastic partial differential equations driven by white noise [127,67,66,97,107]. Such problems, which are not dealt with in this paper, concern numerous applications in physics (diffusion theory...), signal processing (filtering...), optimal control, financial mathematics... Due to the complexity of the input noise, imposing to work in an infinite dimensional probability space, Monte-Carlo simulation technique appears as the most efficient and wide-spread numerical approach for solving such SPDEs. The lack of regularity of the input noise requires the use of specific rules of calculus (e.g. Ito or Stratonovic calculus) and the derivation of specific approximation techniques for solving sample paths (i.e. solving deterministic PDE associated with particular events). Let us also mention methods based the resolution of deterministic PDEs governing the evolution of functionals of the random solution (e.g. Fokker-Planck equation). The use of traditional approximation techniques for solving these PDEs is however limited by the high dimensionality of the problem.

Another class of problems commonly encountered in physical applications concerns the case where the random parameters and the solution of the problem have a finite variance $[34,10,7,9,40,84]$. This class of problems, on which we mainly focus in this paper, occurs when random parameters of the model are represented by variables or random processes (fields) with "nice" regularity properties. The mathematical description of uncertainties a priori requires defining an infinite dimensional probability space. 
However, in numerous physical applications, uncertainty sources can be correctly modeled with a finite set of random variables. This is the case when uncertainties on the model are characterized by a finite set of real-valued random variables or stochastic processes (fields), the latter ones being reduced (or discretized) with ad hoc spectral decomposition techniques (e.g. Karhunen-Loève). Then, in practice, one defines the probabilistic content with a finite set of random variables, defining a new finite dimensional probability space.

In this finite-dimensional framework, numerous computational methods have been proposed for the resolution of a stochastic problem, or more specifically for estimating the probabilistic characterization of a quantity of interest. The choice of a specific method depends on the desired accuracy of the prediction and on the nature of the expected probabilistic information. In particular, if one is interested in first statistical moments of the response (mean, variance,...), perturbation or direct integration methods (Monte-Carlo, Quadrature...) can be used. For the estimation of the probability of particular events, direct integration techniques can still be used as long as the probability of the event remains sufficiently large. For the estimation of small probabilities, specific methodologies, called reliability methods, are generally better adapted. This last decade, a growing interest has been devoted to methods providing a complete characterization of the response (probability law of the quantity of interest), which is represented on a suitable functional expansion basis. These methods, usually named spectral stochastic methods, were initiated by the work of Ghanem and Spanos [48].

An overview of the above mentioned techniques is given in section 2. The subsequent sections mainly focus on spectral stochastic methods. Section 3 introduces classical construction of functional basis for the representation of random variables. In section 4, Galerkin-type spectral stochastic methods, which constitute a particular approach to compute functional representations, are detailed. Issued from a profitable marriage of functional analysis and probability theory, these spectral stochastic methods rely on strong mathematical bases. They lead to highly accurate solutions and allow a better control on numerical simulations: possible construction of a posteriori error estimators, adaptive approximation. These methods seem to constitute one promising way for the numerical simulation of SPDEs. However, several drawbacks slow down the use of these techniques and their application to large scale problems: calculation time, memory requirements, and their intrusive character, which requires a good knowledge of the mathematical structure of the problem and the development of specific solvers for a certain class of problems. In section 5, some recent developments on model reduction techniques are presented, in the context of spectral stochastic methods. Section 6 focuses on the generalized spectral decomposition method, which can be interpreted as an automatic model reduction technique. This method tries to circumvent the above mentioned drawbacks of Galerkin spectral stochastic approaches.

\section{Overview of computational stochastic methods}

In this section, we give a brief overview of classical stochastic methods, emphasize on their domain of applications, their advantages and drawbacks. For complementary reviews on computational stochastic approaches, we refer to $[83,115,60,54,82]$ and the references therein. 
2.1 Generic formulation of stochastic problems

For many physical problems, the conceptual model can be translated in terms of stochastic partial differential equations (SPDEs). The modeling of uncertainties consists in defining a suitable probability space $(\Theta, \mathcal{B}, P)$, where $\Theta$ denotes the space of elementary events, $\mathcal{B}$ a $\sigma$-algebra defined on $\Theta$ and $P$ a probability measure. The response $u$ of the model is then a random variable, with value in a certain function space, which has to verify almost surely a set of equations formally denoted

$$
\mathcal{A}(u(\theta) ; \theta)=b(\theta)
$$

where $\mathcal{A}$ is a differential operator and $b$ a right-hand side associated with the source terms. Uncertainty (or randomness) on the model can be formalized as a dependency of the operator and right-hand side on to the elementary event $\theta \in \Theta$.

In this paper, we consider that the probabilistic content can be correctly modeled with a finite set of random variables $\boldsymbol{\xi}: \theta \in \Theta \mapsto \boldsymbol{\xi}(\theta) \in \boldsymbol{\Xi} \subset \mathbb{R}^{m}$, defining a new finite dimensional probability space $\left(\boldsymbol{\Xi}, \mathcal{B} \boldsymbol{\Xi}, P_{\boldsymbol{\xi}}\right)$, where $\boldsymbol{\Xi}=\boldsymbol{\xi}(\Theta)$, where $\mathcal{B} \boldsymbol{\Xi}$ is a $\sigma$-algebra on $\boldsymbol{\Xi}$ and where $P_{\boldsymbol{\xi}}$ is the probability measure associated with $\boldsymbol{\xi}$ (image measure of $P$ by $\boldsymbol{\xi}$ ). This case is encountered when parameters of operator $\mathcal{A}$ or right-hand side $b$ are real-valued random variables or stochastic processes (or fields), the latter being reduced (or discretized) with ad hoc spectral decomposition techniques (e.g. Karhunen-Loève, see appendix A). A random variable is then be considered as a measurable function defined on $\left(\boldsymbol{\Xi}, \mathcal{B} \boldsymbol{\Xi}, P_{\boldsymbol{\xi}}\right)$. The solution $u$ of the physical model can then be searched as a function of $\boldsymbol{\xi}$, satisfying almost surely a set equations formally written

$$
\mathcal{A}(u(\boldsymbol{\xi}) ; \boldsymbol{\xi})=b(\boldsymbol{\xi})
$$

\subsection{Direct integration techniques}

In a probabilistic analysis, quantities of interest can often be written as the expectation of a certain functional of the response $u$ :

$$
E(f(u(\boldsymbol{\xi}) ; \boldsymbol{\xi})) .
$$

The estimation of such a quantity requires the computation of an integral with respect to measure $P_{\xi}$ :

$$
E(f)=\int_{\boldsymbol{\Xi}} f(u(\mathbf{y}) ; \mathbf{y}) d P_{\boldsymbol{\xi}}(\mathbf{y})=\int_{\boldsymbol{\Xi}} f(u(\mathbf{y}) ; \mathbf{y}) p_{\boldsymbol{\xi}}(\mathbf{y}) d \mathbf{y},
$$

where $p_{\boldsymbol{\xi}}$ denotes the probability density function of $\boldsymbol{\xi}$. Several numerical integration techniques can then be used. In practise, these integration techniques lead to the following estimation:

$$
E(f) \approx Q_{K}(f)=\sum_{k=1}^{K} f\left(u\left(\mathbf{y}_{k}\right) ; \mathbf{y}_{k}\right) \omega_{k},
$$

where the $\omega_{k} \in \mathbb{R}$ and the $\mathbf{y}_{k} \in \boldsymbol{\Xi}$ denote the integration weights and points respectively. Direct integration techniques then only ask for the evaluation of the model's 
response for $K$ outcomes $\boldsymbol{\xi}=\mathbf{y}_{k}$ of basic random variables. The computation of these responses $\left\{u\left(\mathbf{y}_{k}\right)\right\}_{k=1}^{K}$ requires the resolution of $K$ uncoupled deterministic problems:

$$
\mathcal{A}\left(u\left(\mathbf{y}_{k}\right) ; \mathbf{y}_{k}\right)=b\left(\mathbf{y}_{k}\right), \quad k=1 \ldots K .
$$

The interest of these approaches is that well mastered deterministic numerical methods can be used. Moreover, deterministic problems being uncoupled, it allows the use of a massive parallelization. However, in order to obtain a good accuracy on $Q_{K}(f)$, the number of points $K$ can be very large and depends on the function to be integrated.

\subsubsection{Monte-Carlo}

Monte-Carlo integration $[22,116]$ consists in choosing for the integration points $K$ independent random samples (in practice pseudo-random samples) of variables $\boldsymbol{\xi}$. Weights are taken equal to $\omega_{k}=\frac{1}{K}$. The estimation $Q_{K}(f)$ is a random variable and the integration error asymptotically tends toward a Gaussian random variable ${ }^{1}$ :

$$
E(f)-Q_{K}(f) \sim K^{-1 / 2} \sigma_{f} N(0,1),
$$

where $\sigma_{f}$ is the standard deviation of $f$. The estimation being random, a prediction is then given with a certain confidence interval. Standard deviation of the estimator equals $K^{-1 / 2} \sigma_{f}$. The convergence rate of this estimator, in $O\left(K^{-1 / 2}\right)$, is independent of the stochastic dimension $m$, which makes possible the use of Monte-Carlo technique in very high stochastic dimension. However, convergence is very slow.

Numerous improvements have been proposed for Monte-Carlo techniques [22]. They rely on a modification of the generated samples (Antithetic variables, Stratified Sampling, Matching Moment methods, ...) or a modification of the function to be integrated (Importance sampling, Control Variates, ...) in order to reduce its variance and therefore to improve the accuracy.

\subsubsection{Quasi Monte-Carlo}

Quasi Monte-Carlo methods $[22,119,88]$ consist in choosing the points $\left\{\mathbf{y}_{k}\right\}_{k=1}^{K}$ from "low discrepency" sequences and to choose for the weights $\omega_{k}=\frac{1}{K}$. We assume that the integration domain is $\boldsymbol{\Xi}=[0,1]^{m}$ and that the measure $P_{\boldsymbol{\xi}}$ is uniform. This is always possible with a change of basic random variables. From Koksma-Hlawka theorem [22], we have the property

$$
\left|E(f)-Q_{K}(f)\right| \leqslant V(f) D_{K}
$$

where $V(f)$ is the total variation of $f$ and where $D_{K}$ is the discrepency ${ }^{2}$ of the sequence $\left\{\mathbf{y}_{k}\right\}_{k=1}^{K}$. A sequence $\left\{\mathbf{y}_{k}\right\}_{k=1}^{K}$ is said quasi-random if $D_{K} \leqslant c \log (K)^{n} K^{-1}$, where $c$ and $n$ are constants which possibly depend on the dimension $m$. Generally, $n=m$, which leads to the classical error estimate of Quasi Monte-Carlo methods:

$$
\left|E(f)-Q_{K}(f)\right| \sim O\left(\log (K)^{m} K^{-1}\right)
$$

$1 N(0,1)$ denotes a zero mean and unit variance Gaussian random variable

2 The discrepency of a sequence can be interpreted as the maximum error for the approximation of the volume of hyper-rectangles of $[0,1]^{m}$ from samples of this sequence [22]. 
If the stochastic dimension is not too large, it then gives a better asymptotic convergence rate than the basic Monte-Carlo method (convergence dominated by the term $K^{-1}$ ). Some choices of quasi-random sequences can be found in [88]. Figure 1 shows the points obtained from Sobol sequence. One can observe a very uniform distribution of the sequence.

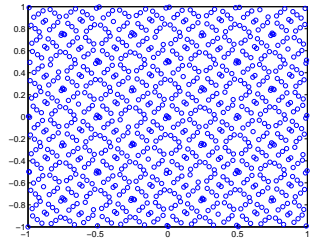

(a) $K=1,000$

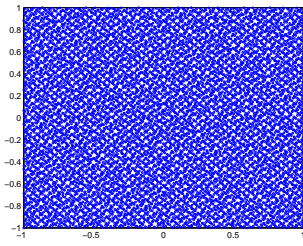

(b) $K=10,000$

Fig. 1 Quasi-Monte Carlo: integration points obtained from Sobol sequence on $\boldsymbol{\Xi}=[0,1]^{2}$ (uniform measure)

\subsubsection{Tensorization of classical quadratures}

$\diamond$ Full tensorization

Classical quadrature techniques (Gauss, Clenshaw-Curtis, ...) can also be used [102]. We here suppose that random variables are independent, so that $\boldsymbol{\Xi}=\times_{i=1}^{m} \Xi_{i}$ and $P_{\boldsymbol{\xi}}=\otimes_{i=1}^{m} P_{\xi_{i}}$. Let us suppose that on each stochastic dimension, a classical quadrature rule $Q^{(i)}$ has been defined:

$$
Q^{(i)}(f)=\sum_{k=1}^{K_{i}} f\left(y_{i, k}\right) \omega_{i, k} \approx \int_{\Xi_{i}} f(y) p_{\xi_{i}}(y) d y .
$$

A quadrature in dimension $m$ can then be obtained by a full tensorization of unidimensional quadratures:

$$
Q_{K}=Q^{(1)} \otimes \ldots \otimes Q^{(m)}
$$

with

$$
Q_{K}(f)=\sum_{k_{1}=1}^{K_{1}} \ldots \sum_{k_{m}=1}^{K_{m}} f\left(y_{1, k_{1}}, \ldots, y_{m, k_{m}}\right) \omega_{1, k_{1}} \ldots \omega_{m, k_{m}}
$$

For quadratures with $K_{i}=n$ points on each dimension, one obtains a total number of points $K=n^{m}$, which increases exponentially with the stochastic dimension. Then, for a function of class $\mathcal{C}^{r}$, the integration error verifies:

$$
\left|E(f)-Q_{K}(f)\right| \sim O\left(K^{-(2 r-1) / m}\right) .
$$

We observe a strong deterioration of the convergence rate in $K$ when increasing the dimension $m$. 
$\diamond$ Smolyak tensorization

Smolyak tensorization formula can be used in order to drastically decrease the number of integration points when dealing with high stochastic dimension $m[118,42,96,100$, 84]. This necessitates the definition of a sequence of quadratures $\left\{Q_{k}^{(i)}\right\}_{k=1}^{l}$ on each dimension, where in $Q_{k}^{(i)}, k$ denotes the level of the quadrature. The idea is to combine one-dimensional quadratures while avoiding the use of high-level quadratures on several dimensions simultaneously. A level $l$ quadrature in dimension $m$ is obtained by the following tensorization formula ${ }^{3}$ :

$$
Q_{K}^{l}=\sum_{\substack{\boldsymbol{k} \in \mathbb{N}^{m} \\
l \leqslant|\boldsymbol{k}| \leqslant l+m-1}}(-1)^{l+m-1-|\boldsymbol{k}|}\left(\begin{array}{c}
l-1 \\
|\boldsymbol{k}|-l
\end{array}\right) Q_{k_{1}}^{(1)} \otimes \ldots \otimes Q_{k_{m}}^{(m)}
$$

If the $Q_{k}^{(i)}$ denote $k$-points quadratures, one obtains a total number of integration points $K \sim O\left(\frac{2^{l}}{l !} m^{l}\right)$. The integration error depends on the smoothness of function $f$. For a $r$-times differentiable function $f$, the integration error behaves as:

$$
\left|E(f)-Q_{K}^{l}(f)\right| \sim O\left(K^{-r} \log (K)^{(m-1)(r+1)}\right) .
$$

We observe a better convergence rate than with the full tensorization. Figures 2 and 3 show integration points obtained with a Smolyak tensorization of Gauss-Hermite and Gauss-Legendre quadratures. These Gaussian quadratures have the property to integrate exactly multidimensional polynomials with total degree less than or equal to $(2 l-1)$ while a full tensorization integrates exactly multidimensional polynomials with partial degree less than or equal to $(2 l-1)$.

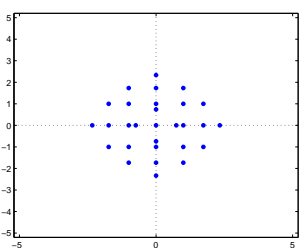

(a) $l=4, K=30$

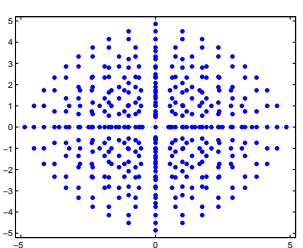

(b) $l=10, K=385$

Fig. 2 Smolyak tensorization of Gauss-Hermite quadratures. Level $l$ quadrature where the $Q_{k}^{(i)}$ are quadratures with $k$ points.

These Smolyak tensorizations are particularly interesting when nested quadratures are used on each dimension (the set of integration points of a quadrature $Q_{k}^{(i)}$ is included in that of $Q_{k+1}^{(i)}$ ). That leads to a significant reduction in the number of integration points. In this case, the obtained integration grids are called "sparse grids". Another major interest of these nested formulas is to reduce the cost of an adaptive integration procedure. Indeed, when increasing the quadrature level, calculations already performed on lower-level grids can be re-used. Several nested quadrature rules are available: Newton-Cotes, Clenshaw-Curtis, Gauss-Patterson...

\footnotetext{
3 Let us note that anisotropic Smolyak tensorization can also be used [43,21].
} 


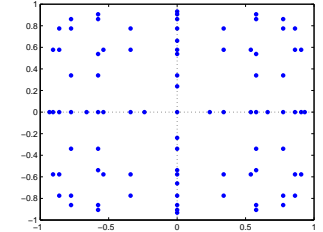

(a) $l=6, K=91$

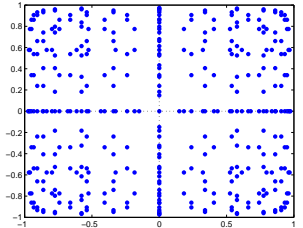

(b) $l=10, K=385$

Fig. 3 Smolyak tensorization of Gauss-Legendre quadratures. Level $l$ quadrature where the $Q_{k}^{(i)}$ are quadratures with $k$ points.

2.3 Perturbation method, Neumann expansions

When one is interested in the first statistical moments of the solution, perturbation or Neumann expansion method are alternative techniques. These methods are based on a series representation of the solution of (2).

\subsubsection{Perturbation method}

The basic perturbation method [65] consists in seeking an expansion of the solution around the mean $\boldsymbol{\mu}_{\xi}=E(\boldsymbol{\xi})$ of random variables:

$$
\begin{aligned}
u(\boldsymbol{\xi}):= & u_{0}+\sum_{i=1}^{m}\left(\xi_{i}-\mu_{\xi_{i}}\right) u_{, i}+ \\
& \sum_{i, j=1}^{m} \frac{1}{2}\left(\xi_{i}-\mu_{\xi_{i}}\right)\left(\xi_{j}-\mu_{\xi_{j}}\right) u_{, i j}+\ldots
\end{aligned}
$$

where $u_{0}:=u\left(\boldsymbol{\mu}_{\boldsymbol{\xi}}\right), u_{, i}:=\frac{\partial u}{\partial \xi_{i}}\left(\boldsymbol{\mu}_{\boldsymbol{\xi}}\right), u_{, i j}:=\frac{\partial^{2} u}{\partial \xi_{i} \xi_{j}}\left(\boldsymbol{\mu}_{\boldsymbol{\xi}}\right), \ldots$ By operating similar expansions for operator $\mathcal{A}(\cdot ; \boldsymbol{\xi})$ and right-hand side $b(\boldsymbol{\xi})$ and by injecting these expansions in equation $\mathcal{A}(u(\boldsymbol{\xi}) ; \boldsymbol{\xi})=b(\boldsymbol{\xi})$, one obtains that the coefficients of the expansion of $u$ are solutions of the following sequence of problems:

$$
\begin{aligned}
& \mathcal{A}_{0}\left(u_{0}\right)=b_{0} \\
& \mathcal{A}_{0}\left(u_{, i}\right)=b_{, i}-\mathcal{A}_{, i}\left(u_{0}\right), \\
& \mathcal{A}_{0}\left(u_{, i j}\right)=b_{, i j}-\mathcal{A}_{, i}\left(u_{, j}\right)-\mathcal{A}_{, j}\left(u_{, i}\right)-\mathcal{A}_{, i j}\left(u_{0}\right),
\end{aligned}
$$

All of these problems are deterministic problems with the same deterministic operator $\mathcal{A}_{0}=\mathcal{A}\left(\cdot ; \boldsymbol{\mu}_{\boldsymbol{\xi}}\right)$. The calculation of the right-hand sides requires to compute the derivatives with respect to variables $\xi_{i}$ of the operator and the right-hand side of the stochastic problem. These quantities, relatively classical in sensitivity analysis, are provided by some computer codes. They generally use numerical differentiation and are often limited to first or second derivatives. Although a priori allowing for a complete representation of the solution, the perturbation method is then often limited to a small order of decomposition (order 2), which limitates its application to the case of basic random variables with of a small coefficient of variation. This method is often used for 
evaluating the first two moments of the solution (mean, covariance), simply expressed in terms of the expansion coefficients:

$$
\begin{aligned}
& E(u)=u_{0}+\frac{1}{2} \sum_{i, j=1}^{m} C_{\xi_{i} \xi_{j}} u_{, i j}+\ldots \\
& E(u \otimes u)=\sum_{i, j=1}^{m} C_{\xi_{i} \xi_{j}}\left(u_{, i} \otimes u_{, j}\right)+\ldots
\end{aligned}
$$

where $C_{\xi_{i} \xi_{j}}$ denotes the covariance of variables $\xi_{i}$ and $\xi_{j}$.

\subsubsection{Neumann decomposition}

Neumann decomposition method $[48,5]$ starts with the following decomposition:

$$
\mathcal{A}(\cdot ; \boldsymbol{\xi})=\mathcal{A}_{0}+\widetilde{\mathcal{A}}(\cdot ; \boldsymbol{\xi})=\mathcal{A}_{0}\left(\mathcal{J}+\mathcal{A}_{0}^{-1} \tilde{\mathcal{A}}(\cdot ; \boldsymbol{\xi})\right),
$$

where $\mathcal{A}_{0}$ is a deterministic operator, $\mathcal{A}_{0}^{-1}$ its inverse, and where $\mathcal{J}$ denotes the identity operator. The inverse of random operator $\mathcal{A}$, under some assumptions [5], can then be written under the form:

$$
\mathcal{A}^{-1}(\cdot ; \boldsymbol{\xi})=\sum_{i=0}^{\infty}(-1)^{i}\left(\mathcal{A}_{0}^{-1} \widetilde{\mathcal{A}}(\cdot ; \boldsymbol{\xi})\right)^{i} \mathcal{A}_{0}^{-1}
$$

so that the solution of problem (2) can be written as:

$$
u(\boldsymbol{\xi})=\sum_{i=0}^{\infty}(-1)^{i} u_{i}(\boldsymbol{\xi})
$$

where the series terms are solutions of the following problems:

$$
\begin{aligned}
& \mathcal{A}_{0}\left(u_{0}(\boldsymbol{\xi})\right)=b(\boldsymbol{\xi}), \\
& \mathcal{A}_{0}\left(u_{i}(\boldsymbol{\xi})\right)=\widetilde{\mathcal{A}}\left(u_{i-1}(\boldsymbol{\xi}) ; \boldsymbol{\xi}\right), \quad \text { pour } i \geqslant 1 .
\end{aligned}
$$

Computing the expansion terms requires the resolution of deterministic problems with random right-hand sides, all these problems being associated with a unique deterministic operator $\mathcal{A}_{0}$. However, these calculations are very expansive [5]. This approach should then preferably be used to estimate the first moments of the solution.

Remark 1 - Connection with the perturbation method.

One can easily show that for a deterministic right-hand side and for an operator $\mathcal{A}(\cdot ; \boldsymbol{\xi})$ which depends linearly in the variables $\xi_{i}$, Neumann expansion method coincides with the perturbation method mentioned in section 2.3.1. 
2.4 Reliability-oriented techniques

In a reliability analysis, the aim is to compute the probability $P_{\boldsymbol{\xi}}(D)$ of a particular event $D$, associated with the failure of a system. In general, the event can be characterized from a quantity of interest $J(u(\boldsymbol{\xi}) ; \boldsymbol{\xi})$, the event "the system fails" corresponding to negative values of this quantity (by convention). The event $D \subset \boldsymbol{\Xi}$, called "failure domain", is defined by $D=\{\mathbf{y} \in \boldsymbol{\Xi} ; J(u(\mathbf{y}) ; \mathbf{y})<0\}$ (see Figure 4 ). The probability of this event is defined by:

$$
P_{\boldsymbol{\xi}}(D)=\int_{D} d P_{\boldsymbol{\xi}}(\mathbf{y})
$$

Various methods have been proposed for the estimation of this probability (see $[36,85]$ for a detailed description) and are already implemented in many commercial codes. We here briefly recall some basic methods.

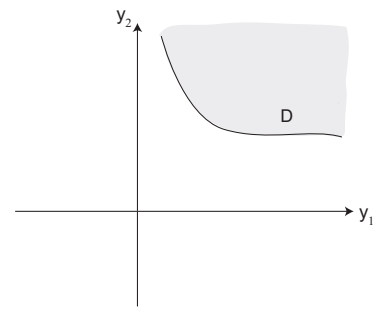

Fig. 4 Failure domain $D$ in $\boldsymbol{\Xi} \subset \mathbb{R}^{2}$.

\subsubsection{First or Second Order Reliability Method (FORM/SORM)}

In the case where the probability $P_{\boldsymbol{\xi}}(D)$ is low (i.e. $D$ is a rare event), methods FORM and SORM consist in approximating the failure domain $D$ by a simpler domain $C$, whose probability $P_{\boldsymbol{\xi}}(C)$ can be estimated analytically. Let us briefly explain the principles of these techniques. We consider that $\xi$ is a vector of independent centered normalized Gaussian random variables (possibly after a suitable change of random variables). We then try to find the most likely failure point $\mathbf{y}^{*} \in D \subset \boldsymbol{\Xi}=\mathbb{R}^{m}$, called the "conception point". Due to the form of standard Gaussian probability density function $p_{\boldsymbol{\xi}}$, this is the point in the failure domain which is the nearest from the origin. It is defined with the following optimization problem:

$$
\mathbf{y}^{*}=\underset{\mathbf{y} \in D}{\operatorname{argmin}}\|\mathbf{y}\|^{2}=\underset{\substack{\mathbf{y} \in \mathbb{R}^{m} \\ J(u(\mathbf{y}) ; \mathbf{y})<0}}{\operatorname{argmin}}\|\mathbf{y}\|^{2} .
$$

Dedicated optimization algorithms have been proposed for the resolution of this problem. For simple failure domains $D$, these algorithms converge rapidly. They ask for the evaluation of functional $J(u(\mathbf{y}) ; \mathbf{y})$ in a few points $\mathbf{y}$, associated with particular outcomes of $\boldsymbol{\xi}$.

The conception point being computed, the FORM method consists in defining the hyper-plane that passes through this point and which is orthogonal to vector $\mathbf{y}^{*}$. This 
hyper-plane defines a semi-space $C$ approximating the domain $D$ (see Figure 5(a)). The probability of $C$ is simply obtained by $P_{\boldsymbol{\xi}}(C)=\Phi(-\beta)$, where $\Phi$ is the standard Gaussian cumulative distribution function and where $\beta$ is the reliability index, defined by $\beta= \pm\left\|\mathbf{y}^{*}\right\|$ (signed distance between the origin and the conception point). In the standard SORM method, a better approximation is provided by introducing a semispace $C$ defined from the paraboloid tangent to $D$ at $\mathbf{y}^{*}$ (Figure $5(\mathrm{~b})$ ). The probability of failure can then be estimated by $P_{\boldsymbol{\xi}}(C)=\Phi(-\beta) \prod_{i=1}^{m}\left(1-\kappa_{i} \beta\right)^{-1 / 2}$, where the $\kappa_{i}$ denote the principal curvatures of the paraboloid.

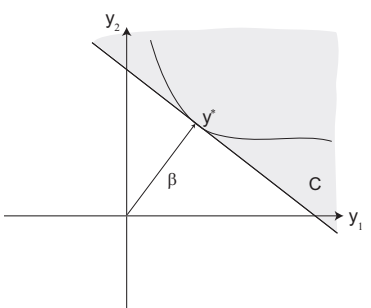

(a) FORM

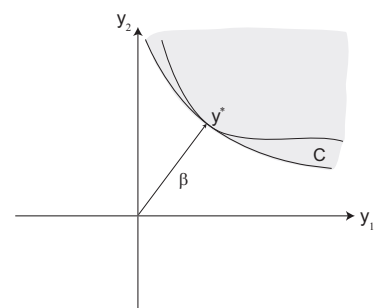

(b) SORM

Fig. 5 FORM (a) and SORM (b) method : approximation $C$ of the failure domain $D$ and reliability index $\beta$

In the case of a relatively small stochastic dimension, FORM and SORM methods allow obtaining relatively accurate predictions with acceptable computational times (reduced number of calls to deterministic codes). However, they have some limitations. In particular, they do not allow to quantify the error on the estimation of the probability of failure. Moreover, in their simplest forms, they are not adapted to complex geometries (or topologies) of failure domains (optimization problem (13) may have several local minima) and can lead to a bad estimation of the probability of failure (especially in high stochastic dimension where FORM and SORM approximations deteriorate).

\subsubsection{Monte-Carlo, Importance Sampling}

A direct integration technique can also be used (see Section 2.2) by interpreting the computation of $P_{\boldsymbol{\xi}}(D)$ by the computation of an expectation:

$$
P_{\boldsymbol{\xi}}(D)=\int_{\boldsymbol{\Xi}} 1_{D}(\mathbf{y}) d P_{\boldsymbol{\xi}}(\mathbf{y})=E\left(1_{D}(\boldsymbol{\xi})\right),
$$

where $1_{D}$ if the indicator function of domain $D$. These techniques have the advantage to give a controlled estimation of the probability.

The use of a standard Monte-Carlo method appears to be prohibitive. One can understand it by observing Figure 6(a), which illustrates that a large number of MonteCarlo samplings are necessary in order to obtain enough samples in $D$. The coefficient of variation of the Monte-Carlo estimator $Q_{K}\left(1_{D}\right)$ is of the order $\left(K P_{\boldsymbol{\xi}}(D)\right)^{-1 / 2}$ for low probability $P_{\boldsymbol{\xi}}(D)$. In order to obtain a desired coefficient of variation $\epsilon$ of the estimator, a very large number of samples $K \approx \epsilon^{-2} P_{\boldsymbol{\xi}}(D)^{-1}$ must be computed (e.g. $K \approx 10^{6}$ for $\epsilon=10^{-1}$ and $P_{\boldsymbol{\xi}}(D)=10^{-4}$ ). 
A possible improvement consists in using the Importance Sampling method. This method consists in rewriting the expectation $E\left(1_{D}(\boldsymbol{\xi})\right)$ as follows:

$$
\begin{aligned}
E\left(1_{D}(\boldsymbol{\xi})\right) & =\int_{\boldsymbol{\Xi}} 1_{D}(\mathbf{y}) \frac{p_{\boldsymbol{\xi}}(\mathbf{y})}{p_{\boldsymbol{\eta}}(\mathbf{y})} p_{\boldsymbol{\eta}}(\mathbf{y}) d \mathbf{y} \\
& =E\left(1_{D}(\boldsymbol{\eta}) \frac{p_{\boldsymbol{\xi}}(\boldsymbol{\eta})}{p_{\boldsymbol{\xi}}(\boldsymbol{\eta})}\right):=E(g(\boldsymbol{\eta})),
\end{aligned}
$$

where $p_{\boldsymbol{\eta}}$ is a well-chosen probability density function allowing to obtain a variance of $g(\boldsymbol{\eta})$ lower than the variance of $1_{D}(\boldsymbol{\xi})$. The classical Monte-Carlo method is then applied to estimate $E(g(\boldsymbol{\eta}))$, by using pseudo-random samples of random variables $\boldsymbol{\eta}$ with probability density function $p_{\boldsymbol{\eta}}$. In the case of a reliability analysis, a possible choice consists in finding the conception point $\mathbf{y}^{*}$ defined by (13) and to define the density $p_{\boldsymbol{\eta}}(\mathbf{y})=p_{\boldsymbol{\xi}}\left(\mathbf{y}-\mathbf{y}^{*}\right)$. In other words, this can be interpreted as a Monte-Carlo method where random samples are centered around the conception point (Figure 6(b)). Various improvements of this Importance Sampling method have been proposed: AxisOrthogonal Importance Sampling, Adaptive Importance Sampling, Radial Importance Sampling...

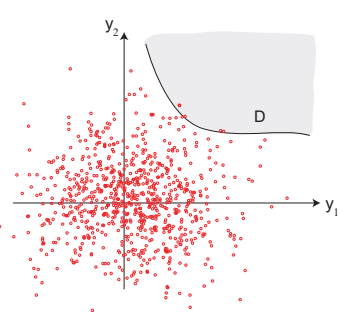

(a) Monte-Carlo

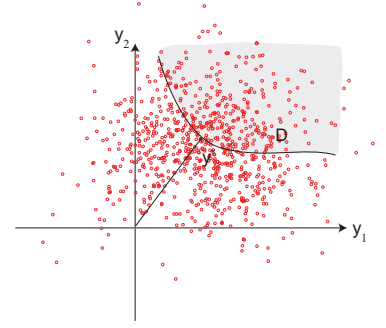

(b) Importance Sampling

Fig. 6 Simulation methods: standard Monte-Carlo (a), Important Sampling around the conception point $\mathbf{y}^{*}$

\subsection{Spectral stochastic methods}

The methods presented above are classically used for evaluating predictions such that moments of a quantity of interest, or the probability of particular events. The application of these methods are limited by more or less restrictive assumptions (variability of input variables, linearity of the problem...) but mainly by computational costs induced by the evaluation of the model response for a large number of outcomes of input variables.

An alternative approach consists in searching a functional representation of solution $u$, or more specifically of a quantity of interest $J(u(\xi) ; \boldsymbol{\xi})$, under the form of a development

$$
J(u(\boldsymbol{\xi}) ; \boldsymbol{\xi}) \approx \sum_{\alpha \in \mathcal{J}_{P}} J_{\alpha} H_{\alpha}(\boldsymbol{\xi}),
$$


where $\left\{H_{\alpha}(\boldsymbol{\xi})\right\}_{\alpha \in \mathcal{J}_{P}}$ is a given basis of functions and where the $J_{\alpha}$ are the coefficients to be determined. This approach can be interpreted as the construction of a response surface of the model. The functional representation (14) being known, a fast post-treatment of the solution can be performed (evaluation of random samples, gradients...). Classical and generic approaches can then be used in order to obtain statistical moments, sensitivity to input variables or even a complete and accurate description of the probability law of $J$.

Since the works of Ghanem and Spanos [48], a growing interest has been devoted to this type of approach, commonly called "spectral stochastic methods". These methods only differ by the choice of basis functions and by the definition (and therefore the computation) of the coefficients of the decomposition. The following sections are devoted to these approaches. Possible choices for basis functions will be presented in section 3. For the definition of the approximation, two classes of approaches may be distinguished: direct approaches ( $L^{2}$ projection, regression, interpolation) and Galerkin-type stochastic approaches.

\subsubsection{Galerkin spectral stochastic methods}

Galerkin-type spectral stochastic approaches $[48,6,84]$ rely on the same mathematical basis as deterministic Galerkin-type approaches. They generally allow for a good control of the approximation (a priori error estimation [34,40], a posteriori error estimation and adaptivity $[62,69,81])$ and are often more robust and definitely more efficient than direct approaches. However, they often require a good knowledge of the mathematical structure of the physical model and the elaboration of specific solvers for a given class of problems.

Many works have been devoted to the elaboration of efficient solvers (see section (4.4)) and more recently to alternative model reduction techniques (see sections 5 and 6). These developments allow drastic reduction of computational costs and make of Galerkin stochastic approaches very efficient and predictive tools. These methods have been applied to various domain of applications in physics (mechanics, chemistry, electromagnetism, ...). Their transfer toward industrial applications, currently under way for some kinds of problems, will need further improvements in methodologies for allowing an efficient treatment of a large class of problems of interest.

Principles of Galerkin stochastic approaches and classical solution techniques will be detailed in section 4 .

\subsubsection{Direct spectral methods}

Direct spectral stochastic methods (projection, regression or interpolation) use a different definition of the coefficients of the decomposition. The computation of the coefficients only requires the resolution of uncoupled deterministic problems. Therefore, they do not require any specific implementations as long as deterministic codes are available. These approaches can be seen as alternatives to direct methods such as Monte-Carlo techniques. However, as Monte-Carlo techniques, they can require the resolution of a large number of deterministic problems and lead to high computational costs. Below, we briefly introduce the principles, advantages and drawbacks of these approaches. 
$\diamond L^{2}$ Projection

The $L^{2}$ projection method $[49,73,74,104,18]$ consists in defining the approximation (14) as the projection of $J$ on the sub-space of $L^{2}\left(\boldsymbol{\Xi}, d P_{\boldsymbol{\xi}}\right)$ spanned by functions $\left\{H_{\alpha}\right\}_{\alpha \in \mathcal{J}_{P}}$, the projection being defined with respect to the natural inner product in $L^{2}\left(\boldsymbol{\Xi}, d P_{\boldsymbol{\xi}}\right)^{4}$ :

$$
<v, w>_{L^{2}\left(\boldsymbol{\Xi}, d P_{\boldsymbol{\xi}}\right)}=E(v(\boldsymbol{\xi}) w(\boldsymbol{\xi}))=\int_{\boldsymbol{\Xi}} v(\mathbf{y}) w(\mathbf{y}) d P_{\boldsymbol{\xi}}(\mathbf{y})
$$

Denoting by $\|v\|_{L^{2}\left(\boldsymbol{\Xi}, d P_{\boldsymbol{\xi}}\right)}=E\left(v(\boldsymbol{\xi})^{2}\right)^{1 / 2}$ the associated norm, the coefficients are defined by

$$
\left\{J_{\alpha}\right\}_{\alpha \in \mathcal{J}_{P}}=\underset{\left\{J_{\alpha}\right\}_{\alpha \in \mathcal{J}_{P}}}{\operatorname{argmin}}\left\|J-\sum_{\alpha \in \mathcal{J}_{P}} J_{\alpha} H_{\alpha}\right\|_{L^{2}\left(\boldsymbol{\Xi}, d P_{\boldsymbol{\xi}}\right)}^{2} .
$$

The use of orthonormal basis functions $\left\{H_{\alpha}\right\}$ leads to the following definition of the coefficients:

$$
J_{\alpha}=<J, H_{\alpha}>_{L^{2}\left(\boldsymbol{\Xi}, d P_{\boldsymbol{\xi}}\right)}=E\left(J(u(\boldsymbol{\xi}) ; \boldsymbol{\xi}) H_{\alpha}(\boldsymbol{\xi})\right) .
$$

The computation of the coefficients then require the evaluation of an integral on $\boldsymbol{\Xi}$ with respect to measure $d P_{\xi}$. For that purpose, one of the integration techniques introduced in section 2.2 can be used, thus leading to the following estimation of the coefficients:

$$
J_{\alpha} \approx \sum_{k=1}^{K} \omega_{k} J\left(u\left(\mathbf{y}_{k}\right) ; \mathbf{y}_{k}\right) H_{\alpha}\left(\mathbf{y}_{k}\right) .
$$

As for direct integration techniques, this approach requires the resolution of $K$ deterministic problems for estimating quantities $J\left(u\left(\mathbf{y}_{k}\right) ; \mathbf{y}_{k}\right)$ associated with particular outcomes $\boldsymbol{\xi}=\mathbf{y}_{k}$. This approach is then usually called a "non-intrusive" projection method in the sense that classical numerical codes can be used to solve these $K$ uncoupled deterministic problems.

This approach, certainly very generic, requires a particular care in the choice of the integration technique. The use of a precise integration is often necessary for obtaining an accurate projection, which requires to solve a very large number of deterministic problems. Of course, a Monte-Carlo integration can be used. However, for an accurate computation of the coefficients, it may require a large number of samples due to the high variance of function $\left(J(u(\boldsymbol{\xi}) ; \boldsymbol{\xi}) H_{\alpha}(\boldsymbol{\xi})\right)$. Therefore, we generally opt for standard high order quadrature techniques (sparse quadrature for high stochastic dimensions $[61,84])$ in order to reduce the number of integration points. The regularity of function $\left(J H_{\alpha}\right)$ being unknown a priori, it is often necessary to use adaptive quadrature techniques (nested quadrature should preferably be used in order to re-use already performed computations). The development of adaptive integration strategies and the definition of rigorous error estimators on the obtained functional representation are still challenges in the context of direct projection techniques. More generally, the lack of rigorous error estimators is a drawback which is common to non-intrusive spectral stochastic techniques (projection, regression, interpolation).

\footnotetext{
${ }^{4}$ See section 3.1.1 for the definition of Hilbert space $L^{2}\left(\boldsymbol{\Xi}, d P_{\boldsymbol{\xi}}\right)$.
} 
$\diamond$ Regression

Regression methods $[29,28,15,124]$ rely on the same principles as classical response surface methods [64]. They consist in defining the coefficient of the decomposition with the following optimization problem:

$$
\min _{\left\{J_{\alpha}\right\}_{\alpha \in \mathcal{J}_{P}}} \sum_{k=1}^{K} \omega_{k}\left(J\left(u\left(\mathbf{y}_{k}\right) ; \mathbf{y}_{k}\right)-\sum_{\alpha \in \mathcal{J}_{P}} J_{\alpha} H_{\alpha}\left(\mathbf{y}_{k}\right)\right)^{2}
$$

where the $\omega_{k}$ and $\mathbf{y}_{k}$ are the regression weights and points respectively. Denoting $\mathrm{H}=\left(\ldots J_{\alpha} \ldots\right) \in \mathbb{R}^{P}$ the set of coefficients to be determined, the optimization problem leads to the following linear system of equations:

$$
\begin{aligned}
& \mathrm{HJ}=\mathrm{Z} \text {, } \\
& (\mathbf{H})_{\alpha \beta}=\sum_{k=1}^{K} \omega_{k} H_{\alpha}\left(\mathbf{y}_{k}\right) H_{\beta}\left(\mathbf{y}_{k}\right), \\
& (\mathbf{Z})_{\alpha}=\sum_{k=1}^{K} \omega_{k} H_{\alpha}\left(\mathbf{y}_{k}\right) J\left(u\left(\mathbf{y}_{k}\right) ; \mathbf{y}_{k}\right) \text {. }
\end{aligned}
$$

Regression methods, as the $L^{2}$ projection, then only require the resolution of uncoupled deterministic problems (associated with different outcomes $\boldsymbol{\xi}=\mathbf{y}_{k}$ ) in order to build the right-hand side of system (19). The choice of regression weights and points is however a diffucult point, especially since the regularity of function $J$ is not known a priori. Several choices have been proposed: Monte-Carlo or Quasi Monte-Carlo samplings for the $\mathbf{y}_{k}$ and $\omega_{k}=\frac{1}{K}$, Gaussian quadrature points and weights for the $\mathbf{y}_{k}$ and $\omega_{k}$ (or a subset of these points), ... The reader can refer to [14] for a detailed study of these different choices (accuracy of the solution, impact on the condition number of the linear system, ...). A methodology for the adaptive construction of functional basis have been proposed in [17].

Remark 2 - Connection with the projection method.

Most of the proposed choices for the $\mathbf{y}_{k}$ and $\omega_{k}$ correspond to points and weights of classical integration techniques. By introducing the "numerical inner product"

$$
<v, w>_{K}:=\sum_{k=1}^{K} \omega_{k} v\left(\mathbf{y}_{k}\right) w\left(\mathbf{y}_{k}\right)
$$

and the associated norm $\|v\|_{K}=<v, v>_{K}^{1 / 2}$, one can interpret the regression problem (18) as the projection of $J$ on span $\left\{H_{\alpha}\right\}_{\alpha \in \mathcal{J}_{P}} \subset L^{2}\left(\boldsymbol{\Xi}, d P_{\boldsymbol{\xi}}\right)$ with respect to inner product $\langle\cdot, \cdot\rangle_{K}$. In fact, coefficients $(\mathrm{H})_{\alpha \beta}$ of matrix $\mathrm{H}$ are approximations of inner products of basis functions: $(\mathrm{H})_{\alpha \beta}=<H_{\alpha}, H_{\beta}>_{K}$. If the quadrature integrates exactly these quantities, we then have a complete equivalence with a classical $L^{2}$ projection method. 
$\diamond$ Interpolation/Collocation

Interpolation techniques [8] consist in choosing for $\left\{H_{\alpha}\right\}_{\alpha \in \mathcal{J}_{P}}$ an interpolation basis on a set of points $\left\{\mathbf{y}_{\alpha}\right\}_{\alpha \in \mathcal{J}_{P}}$. Coefficient $u_{\alpha}$ of the decomposition of $u$ is then obtained by solving a deterministic problem associated with the outcome $\boldsymbol{\xi}=\mathbf{y}_{\alpha}$. The resulting methodology is also called a stochastic collocation approach. For dealing with high stochastic dimension, Smolyak tensorization of unidimensional interpolation basis have been proposed in [39]. The interpolation property of the resulting multidimensional polynomial basis is preserved if nested interpolation grids are used in the Smolyak tensorization. Let us note that when using for the interpolation basis the Lagrange polynomials associated with the points of the classical Gaussian quadrature, the obtained decomposition coincides with the one obtained with a $L^{2}$ projection method associated with this quadrature.

\section{Functional representation of random variables}

For a given physical model, when uncertainties are modeled with a finite set of random variables $\boldsymbol{\xi}=\left(\xi_{1}, \ldots, \xi_{m}\right)$, one has to work on the associated finite dimensional probability space $\left(\boldsymbol{\Xi}, \mathcal{B} \boldsymbol{\Xi}, d P_{\boldsymbol{\xi}}\right)$, where $\boldsymbol{\Xi} \subset \mathbb{R}^{m}$. A random quantity of interest is then interpreted as a random variable defined on $\left(\boldsymbol{\Xi}, \mathcal{B} \boldsymbol{\Xi}, d P_{\boldsymbol{\xi}}\right)$ or in other terms as a measurable function defined on this measured space. For many physical problems, quantities of interest are second order random variables (i.e. with finite second order moments), which leads to introduce the space of square integrable functions $L^{2}\left(\boldsymbol{\Xi}, d P_{\boldsymbol{\xi}}\right)$. This functional point of view of quantities of interest allows proposing functional representation techniques inspired from classical results in functional analysis and approximation theory. Letting $\left\{H_{\alpha}\right\}_{\alpha \in \mathcal{J}_{P}}$ be an approximation basis of $L^{2}\left(\boldsymbol{\Xi}, d P_{\boldsymbol{\xi}}\right)$, one can approximate a quantity of interest $v(\boldsymbol{\xi})$ under the form:

$$
v(\boldsymbol{\xi})=\sum_{\alpha \in \mathcal{J}_{P}} v_{\alpha} H_{\alpha}(\boldsymbol{\xi}) .
$$

Several choices have been proposed for the construction of approximation basis in $L^{2}\left(\boldsymbol{\Xi}, d P_{\boldsymbol{\xi}}\right)$. Classical choices rely on classical construction of polynomial basis (polynomial chaos [130,23,48], generalized polynomial chaos [132], Lagrange interpolation [8]) or piecewise polynomial functions (finite elements [34,128], wavelets [71,72]). A generic vision of this construction and the extension to arbitrary measures is introduced in [121]. The choice of a specific representation depends on regularity properties of functions to be represented. Some choices (finite elements, wavelets) are suitable for developing adaptive approximation techniques.

\subsection{General principles}

\subsubsection{Hilbert space of square integrable functions}

The space of real-valued square integrable functions defined on probability space $\left(\boldsymbol{\Xi}, \mathcal{B} \boldsymbol{\Xi}, P_{\boldsymbol{\xi}}\right)$ (or equivalently the space of real-valued second order random variables) is defined by:

$$
\begin{aligned}
L^{2}\left(\boldsymbol{\Xi}, d P_{\boldsymbol{\xi}}\right)=\{v: \boldsymbol{\xi} \in \boldsymbol{\Xi} \mapsto v(\boldsymbol{\xi}) \in \mathbb{R} ; \\
\left.E\left(v^{2}\right):=\int_{\boldsymbol{\Xi}} v(\mathbf{y})^{2} d P_{\xi}(\mathbf{y})<\infty\right\} .
\end{aligned}
$$


If endowed with the following natural inner product, it is a Hilbert space:

$$
<v, w>_{L^{2}\left(\boldsymbol{\Xi}, d P_{\boldsymbol{\xi}}\right)}=E(v w)=\int_{\boldsymbol{\Xi}} v(\mathbf{y}) w(\mathbf{y}) d P_{\boldsymbol{\xi}}(\mathbf{y})
$$

A Hilbertian basis $\left\{H_{\alpha}\right\}_{\alpha \in \mathcal{J}}$ of $L^{2}\left(\boldsymbol{\Xi}, d P_{\boldsymbol{\xi}}\right)$ is a complete set of orthonormal functions:

$$
\begin{aligned}
& <H_{\alpha}, H_{\beta}>_{L^{2}\left(\boldsymbol{\Xi}, d P_{\boldsymbol{\xi}}\right)}=\delta_{\alpha \beta} \\
& \forall v \in L^{2}\left(\boldsymbol{\Xi}, d P_{\boldsymbol{\xi}}\right),<H_{\alpha}, v>=0, \forall \alpha \in \mathcal{J} \Rightarrow v=0 .
\end{aligned}
$$

Each function $v \in L^{2}\left(\boldsymbol{\Xi}, d P_{\boldsymbol{\xi}}\right)$ admits a unique decomposition on such a basis:

$$
\begin{aligned}
v & =\sum_{\alpha \in \mathcal{J}} v_{\alpha} H_{\alpha}, \\
v_{\alpha} & =<v, H_{\alpha}>_{L^{2}\left(\boldsymbol{\Xi}, d P_{\boldsymbol{\xi}}\right)}=E\left(v(\boldsymbol{\xi}) H_{\alpha}(\boldsymbol{\xi})\right) \\
& =\int_{\boldsymbol{\Xi}} v(\mathbf{y}) H_{\alpha}(\mathbf{y}) d P_{\boldsymbol{\xi}}(\mathbf{y}) .
\end{aligned}
$$

3.1.2 Case of independent basic random variables: tensorization of basis

In the case where random variables $\xi_{i}$ are mutually independent, the construction of approximation basis can be reduced to a one-dimensional construction. Indeed, denoting $\left(\Xi_{i}, \mathcal{B}_{\Xi_{i}}, P_{\xi_{i}}\right)$ the one-dimensional probability space associated with random variable $\xi_{i}$ (where $P_{\xi_{i}}$ is the marginal probability measure associated with $\xi_{i}$ ), we have

$$
\begin{aligned}
& \boldsymbol{\Xi}=\Xi_{1} \times \ldots \times \Xi_{m}, \\
& P_{\boldsymbol{\xi}}=\otimes_{i=1}^{m} P_{\xi_{i}}, \\
& L^{2}\left(\boldsymbol{\Xi}, d P_{\boldsymbol{\xi}}\right)=\otimes_{i=1}^{m} L^{2}\left(\Xi_{i}, d P_{\xi_{i}}\right) .
\end{aligned}
$$

Basis of $L^{2}\left(\boldsymbol{\Xi}, d P_{\boldsymbol{\xi}}\right)$ can then be obtained by tensorization of basis of spaces $L^{2}\left(\Xi_{i}, d P_{\xi_{i}}\right)$. Denoting $\left\{h_{n}^{(i)}\right\}_{n \in \mathcal{J}^{(i)}}$ a basis of $L^{2}\left(\Xi_{i}, d P_{\xi_{i}}\right)$, we let

$$
H_{\alpha}(\mathbf{y})=h_{\alpha_{1}}^{(1)}\left(y_{1}\right) \ldots h_{\alpha_{m}}^{(m)}\left(y_{m}\right)
$$

with $\alpha=\left(\alpha_{1}, \ldots, \alpha_{m}\right) \in \mathcal{J}=\mathcal{J}^{(1)} \times \ldots \times \mathcal{J}^{(m)}$. If basis functions $\left\{h_{n}^{(i)}\right\}_{n \in \mathcal{J}^{(i)}}$ are orthonormal with respect to the natural inner product in $L^{2}\left(\Xi_{i}, d P_{\xi_{i}}\right)$, basis functions $\left\{H_{\alpha}\right\}_{\alpha \in \mathcal{J}}$ are orthonormal:

$$
\begin{aligned}
<H_{\alpha}, H_{\beta}>_{L^{2}\left(\boldsymbol{\Xi}, d P_{\boldsymbol{\xi}}\right)} & =\prod_{i=1}^{m}<h_{\alpha_{i}}^{(i)}, h_{\beta_{i}}^{(i)}>_{L^{2}\left(\Xi_{i}, d P_{\xi_{i}}\right)} \\
& =\prod_{i=1}^{m} \delta_{\alpha_{i} \beta_{i}}:=\delta_{\alpha \beta} .
\end{aligned}
$$


3.1.3 Case of dependent basic random variables

In the case where random variables $\xi_{i}$ are dependent, the above construction of orthonormal basis by tensorization of unidimensional basis is no longer possible since the space $L^{2}\left(\boldsymbol{\Xi}, d P_{\boldsymbol{\xi}}\right)$ has no more a tensor product structure. In [121], the following construction is proposed. Let us define basis $\left\{h_{n}^{(i)}\right\}_{n \in \mathcal{J}(i)}$ of $L^{2}\left(\Xi_{i}, d P_{\xi_{i}}\right)$ as previously. A basis $\left\{H_{\alpha}\right\}_{\alpha \in \mathcal{J}}$ of $L^{2}\left(\boldsymbol{\Xi}, d P_{\boldsymbol{\xi}}\right)$ can then be defined as follows:

$$
H_{\alpha}(\mathbf{y})=h_{\alpha_{1}}^{(1)}\left(y_{1}\right) \ldots h_{\alpha_{m}}^{(m)}\left(y_{m}\right) \sqrt{\frac{p_{\xi_{1}}\left(y_{1}\right) \ldots p_{\xi_{m}}\left(y_{m}\right)}{p_{\boldsymbol{\xi}}\left(y_{1}, \ldots, y_{m}\right)}},
$$

where $p_{\boldsymbol{\xi}}$ is the probability density function of $\boldsymbol{\xi}$ and where $p_{\xi_{i}}$ if the marginal probability density function of $\xi_{i}$.

If basis functions $\left\{h_{n}^{(i)}\right\}_{n \in \mathcal{J}(i)}$ are orthonormal, the orthonormality of basis $\left\{H_{\alpha}\right\}_{\alpha \in \mathcal{J}}$ is preserved. However, even if unidimensional basis functions are polynomials, functions $\left\{H_{\alpha}\right\}$ are no more polynomials in general. Let us note that in the case of independent basic random variables, the proposed construction coincides with the classical construction by tensorization.

3.2 Polynomial approximation

\subsubsection{Spaces of polynomial functions}

The space of multidimensional polynomials with partial degree $p$ defined on $\boldsymbol{\Xi} \subset \mathbb{R}^{m}$ is denoted

$$
\mathbb{Q}_{p}(\boldsymbol{\Xi})=\operatorname{span}\left\{\prod_{i=1}^{m} y_{i}^{\alpha_{i}}, \alpha \in \mathbb{N}^{m} ;|\alpha|_{\infty}:=\max _{i \in\{1 \ldots m\}} \alpha_{i} \leqslant p\right\}
$$

with $\operatorname{dim}\left(\mathbb{Q}_{p}(\boldsymbol{\Xi})\right)=(p+1)^{m}$. The space of multidimensional polynomials of total degree $p$ defined on $\boldsymbol{\Xi} \subset \mathbb{R}^{m}$ is defined by:

$$
\mathbb{P}_{p}(\boldsymbol{\Xi})=\operatorname{span}\left\{\prod_{i=1}^{m} y_{i}^{\alpha_{i}}, \alpha \in \mathbb{N}^{m} ;|\alpha|:=\sum_{i=1}^{m} \alpha_{i} \leqslant p\right\},
$$

with $\operatorname{dim}\left(\mathbb{P}_{p}(\boldsymbol{\Xi})\right)=\frac{(m+p) !}{m ! p !}$. In the case $m=1, \mathbb{P}_{p}(\Xi)=\mathbb{Q}_{p}(\Xi)$. In the case $m>1$, $\mathbb{P}_{p}(\boldsymbol{\Xi}) \subset \mathbb{Q}_{p}(\boldsymbol{\Xi})$

If $\boldsymbol{\Xi}=\Xi_{1} \times \ldots \times \Xi_{m}, \mathbb{Q}_{p}(\boldsymbol{\Xi})$ is a "full" tensorization of unidimensional polynomial spaces of degree $p$ :

$$
\mathbb{Q}_{p}(\boldsymbol{\Xi})=\mathbb{Q}_{p}\left(\Xi_{1}\right) \otimes \ldots \otimes \mathbb{Q}_{p}\left(\Xi_{m}\right)
$$

The space $\mathbb{P}_{p}(\boldsymbol{\Xi})$ can be interpreted as a partial (or "sparse") tensorization of polynomial spaces $\mathbb{Q}_{p}\left(\Xi_{i}\right)$ :

$$
\mathbb{P}_{p}(\boldsymbol{\Xi})=\sum_{\alpha \in \mathbb{N}^{m},|\alpha|=p} \mathbb{Q} \alpha_{1}\left(\Xi_{1}\right) \otimes \ldots \otimes \mathbb{Q} \alpha_{m}\left(\Xi_{m}\right) .
$$




\subsubsection{Polynomial Chaos}

Polynomial Chaos representation consists in using classical orthonormal polynomial basis of $L^{2}\left(\boldsymbol{\Xi}, d P_{\boldsymbol{\xi}}\right)$. In the case where random variables are independent, basis are obtained by a sparse tensorization of polynomial basis of $L^{2}\left(\Xi_{i}, d P_{\xi_{i}}\right)$ (see section 3.1.2). The polynomial chaos of degree $p$ in dimension $m$ is no more than the space $\mathbb{P}_{p}(\boldsymbol{\Xi})$. The homogeneous chaos of degree $p$ in dimension $m$, denoted $\mathbb{H}_{p}$, is the orthogonal complement of $\mathbb{P}_{p-1}(\boldsymbol{\Xi})$ in $\mathbb{P}_{p}(\boldsymbol{\Xi})$. The space $L^{2}\left(\boldsymbol{\Xi}, d P_{\boldsymbol{\xi}}\right)$ admits the following orthogonal decomposition:

$$
L^{2}\left(\boldsymbol{\Xi}, d P_{\boldsymbol{\xi}}\right)=\oplus_{p \in \mathbb{N}} \mathbb{H}_{p}
$$

Let us see how to construct basis functions. On each dimension, associated with a random variable $\xi_{i}$, we introduce an orthonormal polynomial basis $\left\{h_{n}^{(i)}\right\}_{n \in \mathbb{N}}$ of $L^{2}\left(\Xi_{i}, d P_{\xi_{i}}\right)$, where $h_{n}^{(i)} \in \mathbb{P}_{n}\left(\Xi_{i}\right)$ is a polynomial of degree $n$. These polynomials, for a given probability measure $P_{\xi_{i}}$, are uniquely defined and verify:

$$
<h_{n}^{(i)}, h_{l}^{(i)}>_{L^{2}\left(\Xi_{i}, d P_{\xi_{i}}\right)}=\int_{\Xi_{i}} h_{n}^{(i)}(y) h_{l}^{(i)}(y) d P_{\xi_{i}}(y)=\delta_{n l}
$$

In table 1, some classical probability measures and associated orthogonal polynomials are indicated (see e.g. [132] for a more general introduction to orthogonal polynomials).

\begin{tabular}{cccl}
\hline Law & $\Xi$ & $p_{\xi}(y)$ & Polynomials \\
\hline Uniform & {$[-1,1]$} & $\frac{1}{2}$ & Legendre \\
\hline Gausian & $\mathbb{R}$ & $\frac{1}{\sqrt{2 \pi}} \exp \left(-\frac{y^{2}}{2}\right)$ & Hermite \\
\hline Gamma & {$[0,+\infty]$} & $\frac{1}{\Gamma(a)} y^{a} \exp (-y)$ & Laguerre \\
\hline Beta & {$[-1,1]$} & $\frac{(1+y)^{a-1}(1-y)^{b-1}}{2^{a+b-1} B(a, b)}$ & Jacobi \\
\hline
\end{tabular}

Table 1 Classical probability measures and associated orthogonal polynomials ( $\Gamma$ and $B$ are the Euler Gamma and Beta functions respectively).

An orthonormal basis of $\mathbb{H}_{p}$ is then obtained by tensorization of unidimensional polynomials:

$$
\mathbb{H}_{p}=\operatorname{span}\left\{H_{\alpha}(\mathbf{y})=\prod_{i=1}^{m} h_{\alpha_{i}}^{(i)}\left(y_{i}\right), \alpha \in \mathbb{N}^{m} ;|\alpha|=p\right\}
$$

Figure 7 illustrates three basis functions of the Hermite polynomial chaos in dimension $m=2$ (orthonormal polynomials with respect to the standard Gaussian measure $P_{\boldsymbol{\xi}}$ ).

Remark 3 - In the case of dependent random variables, one can use this construction associated with the technique introduced in section 3.1 .3 in order to construct (non polynomial) basis of $L^{2}\left(\boldsymbol{\Xi}, d P_{\boldsymbol{\xi}}\right)$. 


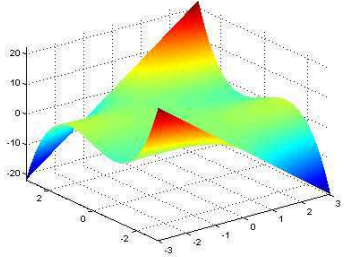

(a) $\alpha=(1,3)$

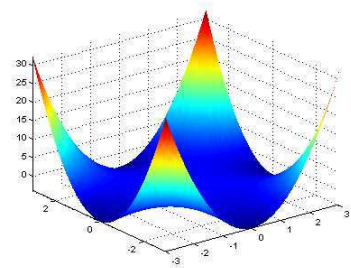

(b) $\alpha=(2,2)$

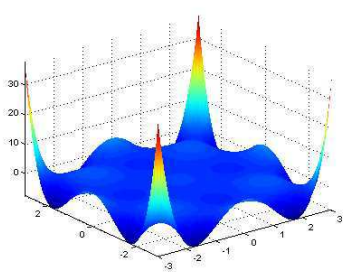

(c) $\alpha=(4,4)$

Fig. 7 Hermite polynomials $H_{\alpha}(\boldsymbol{\xi})$ in dimension $m=2$

\subsubsection{Lagrange Interpolation}

Another approach consists in introducing a basis of $\mathbb{Q}_{p}(\boldsymbol{\Xi})$ (or $\mathbb{P}_{p}(\boldsymbol{\Xi})$ ) composed with interpolation polynomials $[8,39]$. The use of such approximation basis is associated with collocation-type approaches for solving SPDEs.

Here, we suppose that random variables are independent. On each stochastic dimension, we introduce a set of points $\Upsilon_{i}=\left\{y_{i, n}\right\}_{n=0}^{p} \subset \Xi_{i}$ and define the associated interpolation basis $\left\{h_{n}^{(i)}\right\}_{n=0}^{p}$ :

$$
h_{n}^{(i)} \in \mathbb{Q}_{p}\left(\Xi_{i}\right), \quad h_{n}^{(i)}\left(y_{i, l}\right)=\delta_{n l} .
$$

Interpolation points are usually selected as the roots of the classical orthogonal polynomial of degree $(p+1)$, i.e. the $(p+1)$ Gauss points associated with measure $d P_{\xi_{i}}$ (other choices are discussed in [39]). Let us note that this choice leads to orthogonal interpolation functions:

$$
\begin{gathered}
<h_{n}^{(i)}, h_{l}^{(i)}>_{L^{2}\left(\Xi_{i}, d P_{\xi_{i}}\right)}=E\left(h_{n}^{(i)}\left(\xi_{i}\right) h_{l}^{(i)}\left(\xi_{i}\right)\right) \\
=\int_{\Xi_{i}} h_{n}^{(i)}(y) h_{l}^{(i)}(y) d P_{\xi_{i}}(y)=\delta_{n l} \omega_{n},
\end{gathered}
$$

where the $\left\{\omega_{k}\right\}_{k=0}^{p}$ denote Gauss quadrature weights. Indeed, we have

$$
\begin{aligned}
\int_{\Xi_{i}} h_{n}^{(i)}(y) h_{l}^{(i)}(y) d P_{\xi_{i}}(y) & =\sum_{k=0}^{p} \omega_{k} h_{n}^{(i)}\left(y_{i, k}\right) h_{l}^{(i)}\left(y_{i, k}\right) \\
& =\sum_{k=0}^{p} \omega_{k} \delta_{n k} \delta_{l k}=\omega_{n} \delta_{n l} .
\end{aligned}
$$

\section{$\diamond$ Full tensorization}

Basis of $\mathbb{Q}_{p}(\boldsymbol{\Xi})$ can be obtained by a full tensorization of interpolation basis of $\mathbb{Q}_{p}\left(\Xi_{i}\right)$. They are interpolation basis on a multidimensional grid obtained by full tensorization of unidimensional grids $\Upsilon_{i}$. Figures 8 and 9 show interpolation basis functions in dimension $m=2$ obtained with Gaussian and uniform measures respectively. 


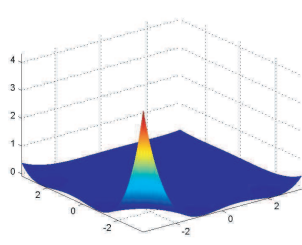

(a) $\alpha=(1,1)$

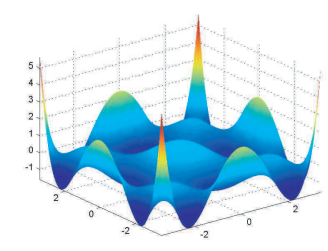

(b) $\alpha=(3,3)$

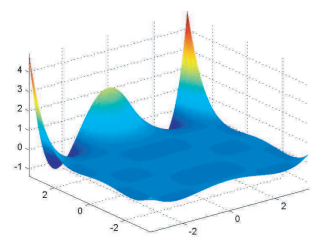

(c) $\alpha=(3,5)$

Fig. 8 Interpolation polynomials $H_{\alpha}(\boldsymbol{\xi}) \in \mathbb{Q}_{4}(\boldsymbol{\Xi})$ on $\boldsymbol{\Xi}=\mathbb{R}^{2}$ : interpolation grid composed by the $5 \times 5$ Gauss-Hermite integration points.

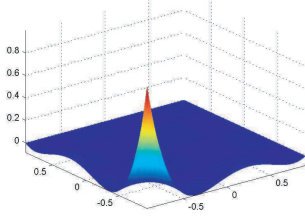

(a) $\alpha=(1,1)$

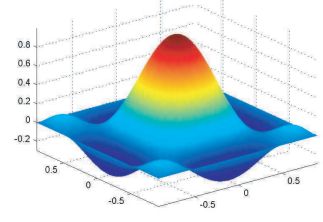

(b) $\alpha=(3,3)$

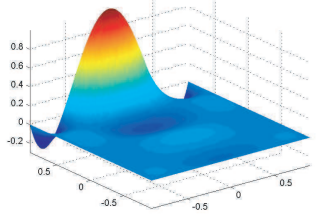

(c) $\alpha=(3,5)$

Fig. 9 Interpolation polynomials $H_{\alpha}(\boldsymbol{\xi}) \in \mathbb{Q}_{4}(\boldsymbol{\Xi})$ on $\boldsymbol{\Xi}=[-1,1]^{2}$ : interpolation grid composed by the $5 \times 5$ Gauss-Legendre integration points.

Remark 4 - Since Gaussian quadrature with $(p+1)$ points exactly integrates polynomials with degree $(2 p+1)$, the following orthogonality property also holds:

$$
\begin{aligned}
E\left(\xi_{i} h_{n}^{(i)}\left(\xi_{i}\right) h_{l}^{(i)}\left(\xi_{i}\right)\right) & =\sum_{k=0}^{p} \omega_{k} y_{i, k} \delta_{n k} \delta_{l k} \\
& =\omega_{n} y_{i, n} \delta_{n l} .
\end{aligned}
$$

This property can be useful in the context of Galerkin-type spectral methods. Indeed, for some kinds of linear problems, one shows that the computation of the decomposition on the interpolation basis can be reduced to a simple stochastic collocation method, which only asks for the resolution of uncoupled deterministic problems (see remark 10 in section 4.3).

\section{$\diamond$ Sparse tensorization.}

Basis of $\mathbb{P}_{p}(\boldsymbol{\Xi})$ can also be obtained by a sparse tensorization of unidimensional interpolation basis, using a Smolyak construction [39]. This construction requires the definition of several interpolation formulas on each stochastic dimension. The obtained multidimensional basis remains interpolatory when nested points are used for unidimensional interpolation formulas (e.g. with Clenshaw-Curtis or Gauss-Patterson points).

\subsection{Piecewise polynomial approximations}

The techniques introduced in $[34,128,71,72]$ consist in choosing piecewise polynomial approximation basis, defined on a partition of $\boldsymbol{\Xi}$. These approximation techniques allow 
representing non-smooth functions, possibly with adaptive approximation procedures $[129,81]$ (by refining the partition or by increasing the approximation degree).

Let us here suppose that domain $\boldsymbol{\Xi}$ is bounded in $\mathbb{R}^{m}$ (which can always be obtained by a suitable change of random variables). We introduce a non-overlapping partition $\left\{\boldsymbol{\Xi}^{k}\right\}_{k=1}^{K}$ of $\boldsymbol{\Xi}$, i.e. such that ${ }^{5}$ :

$$
\cup_{k=1}^{K} \boldsymbol{\Xi}^{k}=\boldsymbol{\Xi}, \quad \boldsymbol{\Xi}^{k} \cap \boldsymbol{\Xi}^{k^{\prime}}=\emptyset \text { if } \quad k \neq k^{\prime} .
$$

An approximation space $\mathcal{S}_{P} \subset L^{2}\left(\boldsymbol{\Xi}, d P_{\boldsymbol{\xi}}\right)$ is defined as the space of functions whose restriction to $\Xi^{k}$ is polynomial of degree $p_{k}$ :

$$
\begin{aligned}
\mathcal{S}_{P} & =\left\{v: \boldsymbol{\Xi} \rightarrow \mathbb{R} ; v_{\mid \boldsymbol{\Xi}^{k}} \in \mathbb{P}_{p_{k}}\left(\boldsymbol{\Xi}^{k}\right)\right\} \\
\text { or } \quad \mathcal{S}_{P} & =\left\{v: \boldsymbol{\Xi} \rightarrow \mathbb{R} ; v_{\mid \boldsymbol{\Xi}^{k}} \in \mathbb{Q}_{p_{k}}\left(\boldsymbol{\Xi}^{k}\right)\right\} .
\end{aligned}
$$

The dimension of this approximation space is $P=\sum_{k=1}^{K} P_{k}$, with $P_{k}=\operatorname{dim}\left(\mathbb{P}_{p_{k}}\right)$ or $\operatorname{dim}\left(\mathbb{Q}_{p_{k}}\right)$. Now, let us see how to construct orthogonal (or orthonormal) basis of $\mathcal{S}_{P}$.

\subsubsection{Classical finite element basis}

$\mathcal{S}_{P}$ can be written as the orthogonal sum of spaces $\mathcal{S}_{P_{k}}^{k}$, where $\mathcal{S}_{P_{k}}^{k}$ if a space of polynomial functions with support $\Xi^{k}$ :

$$
\mathcal{S}_{P}=\oplus_{k=1}^{K} \mathcal{S}_{P_{k}}^{k}, \mathcal{S}_{P_{k}}^{k}=\left\{v \in \mathcal{S}_{P} ; \operatorname{support}(v)=\boldsymbol{\Xi}^{k}\right\} .
$$

An orthonormal basis of $\mathcal{S}_{P}$ can simply be obtained from orthonormal basis $\left\{H_{\alpha}^{k}\right\}_{\alpha \in \mathcal{J}_{P_{k}}}$ of spaces $\mathcal{S}_{P_{k}}^{k} \subset L^{2}\left(\boldsymbol{\Xi}^{k}, d P_{\boldsymbol{\xi}}\right)$. Orthonormality property of the $\left\{H_{\alpha}^{k}\right\}_{\alpha \in \mathcal{J}_{P_{k}}}$ writes:

$$
<H_{\alpha}^{k}, H_{\beta}^{k}>=\int_{\boldsymbol{\Xi}^{k}} H_{\alpha}^{k}(\mathbf{y}) H_{\beta}^{k}(\mathbf{y}) d P_{\xi}(\mathbf{y})=\delta_{\alpha \beta} .
$$

An orthonormal basis of $\mathcal{S}_{P_{k}}^{k}$ can be constructed in a classical way. In the case of independent random variables, an element $\boldsymbol{\Xi}^{k}$ of the partition will be classically defined as an hyper-rectangle $\boldsymbol{\Xi}^{k}=\Xi_{1}^{k} \times \ldots \times \Xi_{m}^{k}$. Then, one obtains an orthonormal polynomial basis of $\mathcal{S}_{P_{k}}^{k}$ by a full or a sparse tensorization of orthonormal basis of $\mathbb{Q}_{p_{k}}\left(\Xi_{i}^{k}\right)$. However, in the general case, the restriction to $\boldsymbol{\Xi}^{k}$ of measure $d P_{\boldsymbol{\xi}}$ is not proportional to a classical probability measure, such that it will not exist a classical orthogonal polynomial basis associated with this restricted measure. Although it is always possible to numerically construct orthogonal basis, it will be easier in practice to use $\boldsymbol{\Xi}=(0,1)^{\mathrm{m}}$ with a uniform measure $P_{\boldsymbol{\xi}}$ (by a suitable change of variables). Indeed, in this case, the restriction of the measure to $\Xi^{k}$ is still a uniform measure and the $H_{\alpha}^{k}$ are simply obtained by tensorization of orthogonal Legendre polynomials.

A simple way to build a partition consists in tensorizing one-dimensional partitions. Denoting $\left\{\Xi_{i}^{k}\right\}_{k=1}^{K_{i}}$ a partition of $\Xi_{i}$, one obtains a regular partition $\left\{\boldsymbol{\Xi}^{k}\right\}_{k=1}^{K}$ of $\boldsymbol{\Xi}$, with $\Xi^{k}=\Xi_{1}^{k_{1}} \times \ldots \times \Xi_{m}^{k_{m}}$. However, the number of elements $K=\prod_{i=1}^{m} K_{i}$ grows exponentially with the stochastic dimension, which can lead to very high-dimensional approximation spaces $\mathcal{S}_{P}\left(\right.$ for $p_{k}=p, P=K \times \operatorname{dim}\left(\mathbb{Q}_{p}\right)$ or $P=K \times \operatorname{dim}\left(\mathbb{P}_{p}\right)$ ). In

\footnotetext{
${ }^{5}$ In probabilistic terms, if $\boldsymbol{\Xi}^{k} \cap \boldsymbol{\Xi}^{k^{\prime}}=\emptyset$, events $\boldsymbol{\Xi}^{k}$ and $\boldsymbol{\Xi}^{k^{\prime}}$ are said incompatible.
} 
an adaptative approximation strategy, this tensorization of one-dimensional partitions does not allow operating local refinements and leads to a rapid increase in the number of elements $K$.

An alternative consists in using octree-type algorithms for partitioning $\boldsymbol{\Xi}$. This approach clearly requires an error estimation criterium and makes sense within an adaptive approximation strategy. The use of anisotropic octree partitions may drastically reduce the number of elements in high stochastic dimension.

\subsubsection{Multi-wavelets}

Another way to define orthonormal basis of $\mathcal{S}_{P}$, proposed in [71,72], consists in using polynomial multi-wavelets basis. This construction has been proposed in the case where $\boldsymbol{\Xi}=(0,1)^{m}$ and $P_{\boldsymbol{\xi}}$ is a uniform measure. A binary partition of $(0,1)^{m}$ is used. Let us briefly explain the construction in the one-dimensional case, i.e. $\boldsymbol{\Xi}=(0,1)$. The obtention of multidimensional basis in $L^{2}\left((0,1)^{m}\right)$ can be obtained by sparse or full tensorizations of unidimensional basis. Let us note $V_{p}^{k}$ the space of piecewise polynomial functions of degree $p$ associated with a binary partition of $(0,1)$ with $2^{k}$ intervals:

$$
\begin{aligned}
V_{p}^{k}=\{v:(0,1) \rightarrow \mathbb{R} ; \forall l & \in\left\{0, \ldots, 2^{k}-1\right\} \\
& \left.v_{\mid\left(2^{-k} l, 2^{-k}(l+1)\right)} \in \mathbb{Q}_{p}\left(\left(2^{-k} l, 2^{-k}(l+1)\right)\right)\right\}
\end{aligned}
$$

Let us note $W_{p}^{k}$ the orthogonal complement of $V_{p}^{k}$ in $V_{p}^{k+1}$ :

$$
V_{p}^{k+1}=V_{p}^{k} \oplus W_{p}^{k}
$$

We then obtain the following decomposition: $L^{2}((0,1))=V_{p}^{0} \oplus_{k \geqslant 0} W_{p}^{k}$. The space $W_{p}^{k}$ is the space of multi-wavelets with resolution $k$. This multi-wavelet vision allows for a multi-scale representation of functions in $L^{2}((0,1))$, spaces $W_{p}^{k}$ being associated with more and more local details as the resolution $k$ increases.

For the construction of an orthonormal basis associated with this decomposition, we first introduce an orthonormal basis $\left\{\phi_{n}\right\}_{n=0}^{p}$ of $V_{p}^{0}$ (composed by Legendre polynomials) and an orthonormal basis $\left\{\psi_{n}\right\}_{n=0}^{p}$ of $W_{p}^{0}$. An orthonormal basis of $W_{p}^{k}$ is then composed with multi-wavelets $\psi_{n, l}^{k}$, defined by:

$$
\psi_{n, l}^{k}(y)=2^{k / 2} \psi_{n}\left(2^{k} y-l\right), l=0 \ldots 2^{k}-1, n=0 \ldots p .
$$

\section{Galerkin-type spectral stochastic methods}

Galerkin-type spectral stochastic methods, briefly discussed in section 2.5.1, rely on a generic procedure for the prediction of the response of a large class of models governed by stochastic partial differential equations (SPDEs). These methods are based on the same principles as deterministic Galerkin methods. They define an approximation of the solution, represented on a certain approximation basis (see Section 3), based on a weak formulation of the stochastic problem (2). After a brief introduction of some aspects of the analysis of SPDEs, we recall in this section the principles of Galerkin stochastic approaches and the associated resolution techniques. 
4.1 Stochastic partial differential equations

\subsubsection{Strong formulation of the problem}

Stochastic partial differential equations can generally be reduced to the research of a random variable $u$, defined on a probability space $\left(\boldsymbol{\Xi}, \mathcal{B} \boldsymbol{\Xi}, d P_{\boldsymbol{\xi}}\right)$, and verifying almost surely a set of equations, formally denoted by:

$$
\mathcal{A}(u(\boldsymbol{\xi}) ; \boldsymbol{\xi})=b(\boldsymbol{\xi}),
$$

where $\mathcal{A}$ is a differential operator, possibly non-linear, and where $b$ denotes a given right-hand side.

\subsubsection{Weak formulation at the deterministic level}

The mathematical analysis of problem (33) and the development of deterministic approximation methods (finite elements, spectral approaches,...) generally start with a weak formulation at the deterministic level of problem (33): find a random variable $u$ with value in a function space $\mathcal{V}$ verifying almost surely:

$$
a(u(\boldsymbol{\xi}), v ; \boldsymbol{\xi})=b(v ; \boldsymbol{\xi}) \quad \forall v \in \mathcal{V},
$$

where $a(\cdot, \cdot ; \boldsymbol{\xi})$ is a semilinear form (eventually bilinear form) on $\mathcal{V} \times \mathcal{V}$ and where $b(\cdot ; \boldsymbol{\xi})$ is a bilinear form on $\mathcal{V}$. The random solution $u(\boldsymbol{\xi})$ of problem (34) is a strong solution at the stochastic level. A classical mathematical analysis [19,105] (analysis of properties of $a$ and $b$ ) allows determining well-posedness of the problem: existence and uniqueness of solution, continuous dependence on the data.

\subsubsection{Weak formulation at the stochastic level}

For a wide class of physical models, the solution is a second order random variable. A solution of (34) can then classically be searched in a subspace of $L^{2}\left(\boldsymbol{\Xi}, d P_{\boldsymbol{\xi}} ; \mathcal{V}\right)$, the space of second order random variables with values in function space $V$ :

$$
L^{2}\left(\boldsymbol{\Xi}, d P_{\boldsymbol{\xi}} ; \mathcal{V}\right)=\left\{v: \boldsymbol{\Xi} \rightarrow \mathcal{V} ; E\left(\|v\|_{\mathcal{V}}^{2}\right)<\infty\right\} .
$$

Here, we suppose that $\mathcal{V}$ is independent of the elementary event ${ }^{6}$. The working function space can then be assimilated to a tensor product space:

$$
L^{2}\left(\boldsymbol{\Xi}, d P_{\boldsymbol{\xi}} ; \mathcal{V}\right) \simeq \mathcal{V} \otimes L^{2}\left(\boldsymbol{\Xi}, d P_{\boldsymbol{\xi}}\right):=\mathcal{V} \otimes \mathcal{S} .
$$

A weak formulation of problem (34) can then be written: find $u \in \mathcal{V} \otimes \mathcal{S}$ such that

$$
A(u, v)=B(v) \quad \forall v \in \mathcal{V} \otimes \mathcal{S},
$$

with

$$
\begin{aligned}
A(u, v) & :=E(a(u(\boldsymbol{\xi}), v(\boldsymbol{\xi}) ; \boldsymbol{\xi})) \\
& =\int_{\boldsymbol{\Xi}} a(u(\mathbf{y}), v(\mathbf{y}) ; \mathbf{y}) d P_{\boldsymbol{\xi}}(\mathbf{y}),
\end{aligned}
$$

6 This hypothesis is not verified for classical formulations of PDE defined on random domains $[95,25,92,122]$ 
and

$$
B(v):=E(b(v(\boldsymbol{\xi}) ; \boldsymbol{\xi}))=\int_{\boldsymbol{\Xi}} b(v(\mathbf{y}) ; \mathbf{y}) d P_{\boldsymbol{\xi}}(\mathbf{y}) .
$$

Well-posedness of problem (35) still results from a similar analysis of forms $A$ and $B$ $[16,5,9,40,84,120]$. One can find a more general framework in $[12,55,16]$, introducing spaces of generalized random variables (distributions spaces).

Remark 5 - Let us notice that some problems may require the introduction of Lebesgue spaces $\mathcal{S}=L^{p}\left(\boldsymbol{\Xi}, d P_{\boldsymbol{\xi}}\right), p \geqslant 2$ (see e.g. [84] for the formulation of nonlinear elliptic problems). From a numerical point of view (see below), classical construction of approximation spaces presented in section 3 may be used (classical results about density of polynomial spaces in $L^{p}$ spaces).

\subsubsection{Model example: stationary diffusion equation}

In order to illustrate the issues outlined above, we consider a classical stationary diffusion problem whose weak formulation writes as in (34), with $\mathcal{V}=H_{0}^{1}(\Omega)$ and

$$
\begin{aligned}
& a(u, v ; \boldsymbol{\xi})=\int_{\Omega} \kappa(x, \boldsymbol{\xi}) \nabla u(x, \boldsymbol{\xi}) \cdot \nabla v(x, \boldsymbol{\xi}) d x, \\
& b(v ; \boldsymbol{\xi})=\int_{\Omega} v(x, \boldsymbol{\xi}) b(x, \boldsymbol{\xi}) d x,
\end{aligned}
$$

where $\kappa(\cdot, \boldsymbol{\xi})$ is a stochastic field. The following condition gives a necessary condition for the well-posedness of the problem in the sense of Hadamard (existence, uniqueness and continuous dependence on the data): if there exist some constants $\kappa_{0}$ and $\kappa_{1}$ such that we have almost surely and almost everywhere on $\Omega$

$$
0<\kappa_{0} \leqslant \kappa(x, \theta) \leqslant \kappa_{1}<\infty
$$

we classically show that $a$ is almost surely continuous and coercive, i.e. there exist strictly positive constants $c_{a}$ and $\alpha_{a}$ such that $\forall u, v \in \mathcal{V}$,

$$
\begin{aligned}
& |a(u, v ; \boldsymbol{\xi})| \leqslant c_{a}\|u\|_{\mathcal{V}}\|v\|_{\mathcal{V}}, \\
& a(v, v ; \boldsymbol{\xi}) \geqslant \alpha_{a}\|v\|_{\mathcal{V}}^{2} .
\end{aligned}
$$

Supposing that the right-hand side of (33) satisfies classical regularity properties ensuring continuity of bilinear form $b(\cdot ; \boldsymbol{\xi})$, we ensure the existence and uniqueness of a strong solution at the stochastic level [9].

The existence and uniqueness of a weak solution to problem (35) is ensured if the following properties are satisfied: $\forall u, v \in \mathcal{V} \otimes \mathcal{S}$,

$$
\begin{aligned}
& |A(u, v)| \leqslant c_{A}\|u\|_{\mathcal{V} \otimes \mathcal{S}}\|v\|_{\mathcal{V} \otimes \mathcal{S}} \\
& A(v, v) \geqslant \alpha_{A}\|v\|_{\mathcal{V} \otimes \mathcal{S}}^{2} \\
& |B(v)| \leqslant c_{B}\|v\|_{\mathcal{V} \otimes \mathcal{S}}
\end{aligned}
$$

In particular, if the stochastic field $\kappa$ verifies property (41), and if there exist constants $\kappa_{0}$ and $\kappa_{1}$ independent of the elementary event $\boldsymbol{\xi}$, we show that coercivity and continuity properties of $A$ follow with the same constants as for a, i.e. $c_{A}=c_{a}$ and $\alpha_{A}=\alpha_{a}$. Condition (41), with $\kappa_{0}$ and $\kappa_{1}$ independent of the elementary event, is necessary and sufficient to obtain a well posed problem in the sense of Hadamard. 
Remark 6 - Typical violation of existence conditions.

When parameters of SPDE are stochastic fields, one generally starts with a discretization of these fields (see appendix A) in order to work in a finite dimensional probability space. A particular care must be taken to this discretization step in order to keep a well-posed problem (34). As an example, let us consider again the stationary diffusion problem. Let $\kappa_{e x}(x, \theta)$ denote the initial stochastic field, defined on a probability space $(\Theta, \mathcal{B}, P)$. A good probabilistic modeling step consists in choosing a stochastic field $\kappa_{\text {ex }}$ satisfying conditions (41), thus ensuring the almost-sure existence of a solution defined on $(\Theta, \mathcal{B}, P)$. The discretization step consists in approximating $\kappa_{e x}(x, \theta)$ by a stochastic field $\kappa(x, \boldsymbol{\xi}(\theta))$, by using classical spectral decomposition techniques (e.g. Karhunen-Loève) or a Polynomial Chaos decomposition. However, for commonly used stochastic fields, these decompositions may only converge in $L^{2}(\Omega \times \Theta)$ and not uniformly. Therefore, after truncation, stochastic field $\kappa(x, \boldsymbol{\xi}(\theta))$ may not verify conditions (41) anymore [5]. A way to circumvent this problem consists in using, if possible, a stochastic field $\kappa_{e x}(x, \theta)=f\left(\gamma_{e x}(x, \theta) ; x\right)$ writing as a nonlinear functional of a Gaussian stochastic field $\gamma_{\text {ex }}$ [52, 45, 103, 60,59, 84], with $f$ allowing to ensure property (41). After discretization and renormalization of the stochastic field $\gamma_{e x}(x, \theta) \approx \gamma(x, \boldsymbol{\xi}(\theta))$, we define an approximate stochastic field $\kappa(x, \boldsymbol{\xi})=f(\gamma(x, \boldsymbol{\xi}) ; x)$ defined on probability space $\left(\boldsymbol{\Xi}, \mathcal{B} \boldsymbol{\Xi}, P_{\boldsymbol{\xi}}\right)$ and verifying conditions (41).

Remark 7 - Other existence results.

In [120], one can find a construction of stochastic fields (in the context of linear elasticity) verifying continuity and ellipticity conditions weaker than " $\kappa_{0}<\kappa(x, \theta)<\kappa_{1}$ almost surely" and still ensuring the uniqueness and existence of solution in $\mathcal{V} \otimes$ $L^{2}\left(\boldsymbol{\Xi}, d P_{\boldsymbol{\xi}}\right)$. These conditions do not require that $\kappa$ is bounded uniformly from above and below (the marginal probability law of $\kappa(x, \cdot)$ may have $\mathbb{R}^{+}$as support). The continuity of bilinear $A$ requires a stronger regularity assumption on the right-hand side, while the ellipticity of $A$ requires some assumptions on the stochastic field $\kappa^{7}$ The reader can refer to [16] for more general existence results, requiring the introduction of new spaces of random variables in order to take into account a larger class of stochastic fields.

4.2 Approximation at the deterministic level

\subsubsection{Strong formulation of the semi-discretized problem}

Classical approximation techniques at the deterministic level (finite difference, finite volume, finite elements...) classically lead to the resolution of the following semidiscretized problem: find a random variable $\mathbf{u}(\boldsymbol{\xi})$ with values in $\mathbb{R}^{n}$ (or a random vector) verifying almost surely:

$$
\mathbf{A}(\mathbf{u}(\boldsymbol{\xi}) ; \boldsymbol{\xi})=\mathbf{b}(\boldsymbol{\xi})
$$

where $\mathbf{A}(\cdot ; \boldsymbol{\xi})$ is a random operator from $\mathbb{R}^{n}$ into $\mathbb{R}^{n}$ and where $\mathbf{b}(\boldsymbol{\xi})$ is a random vector.

7 The weaker ellipticity condition used in [120], writing $\kappa^{-1} \in L^{2}\left(\boldsymbol{\Xi}, d P_{\xi} ; L^{\infty}(\Omega)\right)$ instead of the classical condition $L^{\infty}\left(\boldsymbol{\Xi}, d P_{\boldsymbol{\xi}} ; L^{\infty}(\Omega)\right)$ introduced in $[9,84,40]$, seems not sufficient to obtain a well-posed problem in the classical sense of Hadamard (i.e. in order to guarantee the continuous dependence on the data). This result has to be confirmed. 
Example 1 (Galerkin approaches at the deterministic level) Classical Galerkin approaches (finite element [123,31], spectral methods [24], ...) start from a weak formulation at the deterministic level (equation (34)) and introduce an approximation space $\mathcal{V}_{n} \subset \mathcal{V}$ of dimension $n$. The Galerkin approximation of problem (34), which is a random variable $u_{n}(\boldsymbol{\xi})$ with values in $\mathcal{V}_{n}$, must verify almost surely

$$
a\left(u_{n}(\boldsymbol{\xi}), v_{n} ; \boldsymbol{\xi}\right)=b\left(v_{n} ; \boldsymbol{\xi}\right) \quad \forall v_{n} \in \mathcal{V}_{n}
$$

Denoting by $\left\{\varphi_{i}\right\}_{i=1}^{n}$ a basis of $\mathcal{V}_{n}$ and by $\mathbf{u}=\left(u_{i}\right)_{i=1}^{n}$ the vector of components of $u_{n}$ on this basis, the discretized operator and right-hand side of (47) are naturally defined as:

$$
(\mathbf{A}(\mathbf{u} ; \boldsymbol{\xi}))_{i}=a\left(u_{n}, \varphi_{i} ; \boldsymbol{\xi}\right), \quad(\mathbf{b}(\boldsymbol{\xi}))_{i}=b\left(\varphi_{i} ; \boldsymbol{\xi}\right) .
$$

In the case of a linear problem, $\mathbf{A}$ is a random matrix whose components writes:

$$
(\mathbf{A}(\boldsymbol{\xi}))_{i j}=a\left(\varphi_{j}, \varphi_{i} ; \boldsymbol{\xi}\right)
$$

4.2.2 Weak formulation of the semi-discretized problem

A weak formulation at the stochastic level reads: find $\mathbf{u} \in \mathbb{R}^{n} \otimes \mathcal{S}$ such that

$$
A(\mathbf{u}, \mathbf{v})=B(\mathbf{v}) \quad \forall \mathbf{v} \in \mathbb{R}^{n} \otimes \mathcal{S}
$$

with

$$
\begin{aligned}
& A(\mathbf{u}, \mathbf{v}):=E\left(\mathbf{v}(\boldsymbol{\xi})^{T} \mathbf{A}(\mathbf{u}(\boldsymbol{\xi}) ; \boldsymbol{\xi})\right) \\
& B(\mathbf{v})=E\left(\mathbf{v}(\boldsymbol{\xi})^{T} \mathbf{b}(\boldsymbol{\xi})\right)
\end{aligned}
$$

4.3 Galerkin approximation

\subsubsection{Definition of the approximation}

Galerkin-type spectral stochastic methods start from the weak formulation (49). They introduce an approximation space $\mathcal{S}_{P} \subset \mathcal{S}$ (see section 3) and define the Galerkin approximation $\mathbf{u} \in \mathbb{R}^{n} \otimes \mathcal{S}_{P}$ as follows:

$$
A(\mathbf{u}, \mathbf{v})=B(\mathbf{v}) \quad \forall \mathbf{v} \in \mathbb{R}^{n} \otimes \mathcal{S}_{P},
$$

or equivalently, coming back to the definitions (50) of $A$ and $B$,

$$
\begin{aligned}
& E\left(\mathbf{v}^{T} \mathbf{R}(\mathbf{u})\right)=0 \quad \forall \mathbf{v} \in \mathbb{R}^{n} \otimes \mathcal{S}_{P}, \\
& \text { with } \mathbf{R}(\mathbf{u}(\boldsymbol{\xi}) ; \boldsymbol{\xi})=\mathbf{b}(\boldsymbol{\xi})-\mathbf{A}(\mathbf{u}(\boldsymbol{\xi}) ; \boldsymbol{\xi}) .
\end{aligned}
$$

Equation (52) is equivalent to cancelling the orthogonal projection on $\mathbb{R}^{n} \otimes \mathcal{S}_{P}$ of the residual $\mathbf{R}(\mathbf{u})$. It is equivalent to verify equation (47) in a weak sense. Classical mathematical arguments allow to precise the properties of the Galerkin approximation: convergence, stability, a priori error estimation [12,34,6,40]. In particular, the Galerkin approximation appears to have good stability properties with respect to perturbations and integration error, properties which are very interesting from a numerical point of view. 
Property 1 (Linear symmetric elliptic problem) In the case of a linear symmetric elliptic problem, bilinear form $A$ defines an inner product $<\cdot, \cdot>_{A}$ on $\mathbb{R}^{n} \otimes \mathcal{S}$. The Galerkin approximation is then the projection on $\mathbb{R}^{n} \otimes \mathcal{S}_{P}$ of the solution of problem (49) with respect to inner product $\langle\cdot, \cdot\rangle_{A}$. The approximation then minimizes the distance to the solution of (49), the distance being defined with the norm induced by $A$.

\subsubsection{System of equations}

Denoting $\left\{H_{\alpha}\right\}_{\alpha \in \mathcal{J}_{P}}$ a basis of $\mathcal{S}_{P}$, the solution $\mathbf{u} \in \mathbb{R}^{n} \otimes \mathcal{S}_{P}$ is searched under the form of a decomposition

$$
\mathbf{u}(\boldsymbol{\xi})=\sum_{\alpha \in \mathcal{J}_{P}} \mathbf{u}_{\alpha} H_{\alpha}(\boldsymbol{\xi}), \quad \mathbf{u}_{\alpha} \in \mathbb{R}^{n}
$$

Problem (51) is then equivalent to the following system of equations: $\forall \alpha \in \mathcal{J}_{P}$,

$$
E\left(H_{\alpha}(\boldsymbol{\xi}) \mathbf{A}\left(\sum_{\beta \in \mathcal{J}_{P}} \mathbf{u}_{\beta} H_{\beta}(\boldsymbol{\xi}) ; \boldsymbol{\xi}\right)\right)=E\left(\mathbf{b}(\boldsymbol{\xi}) H_{\alpha}(\boldsymbol{\xi})\right)
$$

This is a system of $n \times P$ equations, possibly non-linear. Denoting $\mathbf{u} \in \mathbb{R}^{n P}$ the block vector gathering components $\mathbf{u}_{\alpha}:=(\mathbf{u})_{\alpha}$, system (53) can be recasted as a block system of equations:

$$
\mathbf{A}(\mathbf{u})=\mathbf{b},
$$

with

$$
\begin{aligned}
& (\mathbf{A}(\mathbf{u}))_{\alpha}=E\left(H_{\alpha} \mathbf{A}\left(\sum_{\beta \in \mathcal{J}_{P}} \mathbf{u}_{\beta} H_{\beta}\right)\right), \\
& (\mathbf{b})_{\alpha}=E\left(\mathbf{b} H_{\alpha}\right) .
\end{aligned}
$$

Remark 8 - Use of piecewise polynomial approximations.

When using finite element-type piecewise polynomial approximations (see section 3.3), the space $\mathcal{S}_{P}$ can be written $\mathcal{S}_{P}=\oplus_{k=1}^{K} \mathcal{S}_{P_{k}}^{k}$, where $\mathcal{S}_{P_{k}}^{k}$ denotes a subspace of polynomial functions having for support an element $\boldsymbol{\Xi}^{k}$ of a partition of $\boldsymbol{\Xi}$. The solution $\mathbf{u}$ can then be written $\mathbf{u}=\sum_{k=1}^{K} \mathbf{u}^{k}$, where the $\mathbf{u}^{k} \in \mathcal{S}_{P_{k}}^{k}$ are defined by $K$ uncoupled problems: $\forall k \in\{1, \ldots, K\}$

$$
A\left(\mathbf{u}^{k}, \mathbf{v}^{k}\right)=B\left(\mathbf{v}^{k}\right) \quad \forall \mathbf{v}^{k} \in \mathbb{R}^{n} \otimes \mathcal{S}_{P_{k}}^{k}
$$

One then have to solve $K$ uncoupled systems of equations of type (53), each system involving the basis functions $\left\{H_{\alpha}^{k}\right\}_{\alpha \in \mathcal{J}_{P_{k}}}$ of $\mathcal{S}_{P_{k}}^{k}$. 
4.3.3 The case of linear problems

In the case of linear problems, system (53) writes:

$$
\sum_{\beta \in \mathcal{J}_{P}} E\left(\mathbf{A} H_{\alpha} H_{\beta}\right) \mathbf{u}_{\beta}=E\left(\mathbf{b} H_{\alpha}\right), \quad \forall \alpha \in \mathcal{J}_{P}
$$

System (54) becomes a system of $n \times P$ linear equations

$$
\mathbf{A u}=\mathbf{b}
$$

where $\mathbf{A}$ is a matrix whose block components write

$$
(\mathbf{A})_{\alpha \beta}=E\left(\mathbf{A} H_{\alpha} H_{\beta}\right) \text {. }
$$

In practice, random matrix $\mathbf{A}$ is decomposed on the basis of functions $\left\{H_{\alpha}\right\}_{\alpha \in \mathcal{J}}$ :

$$
\mathbf{A}(\boldsymbol{\xi})=\sum_{\alpha \in \mathcal{J}_{P_{A}}} \mathbf{A}_{\alpha} H_{\alpha}(\boldsymbol{\xi})
$$

where $\mathcal{J}_{P_{A}} \subset \mathcal{J}$ denotes a finite set of indices. Blocks of matrix $\mathbf{A}$ can then be written

$$
(\mathbf{A})_{\alpha \beta}=\sum_{\gamma \in \mathcal{J}_{P_{A}}} \mathbf{A}_{\gamma} E\left(H_{\gamma} H_{\alpha} H_{\beta}\right) \text {. }
$$

For some classical stochastic basis functions, the terms $E\left(H_{\gamma} H_{\alpha} H_{\beta}\right)$ are often known analytically. They can also be pre-computed numerically.

Remark 9 - Truncation of the decomposition of the operator.

Random matrix A a priori admits a convergent decomposition on the complete basis $\left\{H_{\alpha}\right\}_{\alpha \in \mathcal{J}}$ of $L^{2}\left(\boldsymbol{\Xi}, d P_{\boldsymbol{\xi}}\right)$. By truncating this decomposition to the subset $\mathcal{J}_{P_{A}}$, one a priori introduces an approximation of the operator (variational crime), which could lead to a solution different from the solution of the initial problem (51). In fact, one can easily show that if $\left\{H_{\alpha}\right\}_{\alpha \in \mathcal{J}}$ is an orthogonal (piecewise) polynomial basis and if $\mathcal{S}_{P}$ corresponds to polynomials with degree $p$, a decomposition of $\mathbf{A}$ on a polynomial basis of degree $2 p$ is sufficient to obtain the solution of the initial problem. Indeed, from orthogonality of basis functions,

$$
\begin{aligned}
E\left(\mathbf{A} H_{\alpha} H_{\beta}\right) & =\sum_{\gamma \in \mathcal{J}} \mathbf{A}_{\gamma} E\left(H_{\gamma} H_{\alpha} H_{\beta}\right) \\
& =\sum_{\gamma \in \mathcal{J}_{P_{A}}} \mathbf{A}_{\gamma} E\left(H_{\gamma} H_{\alpha} H_{\beta}\right) .
\end{aligned}
$$

Property 2 (Taking into account exactly stochastic fields) When a stochastic field intervenes in the definition of the operator of the initial probabilistic model, one generally has to perform a discretization of the field in order to work in a finite-dimensional probability space. In fact, one can show that the Galerkin approach allows to take into account exactly the initial stochastic field. Let us illustrate this property on the example of section 4.1.4. Let us first consider the problem with the initial stochastic 
field $\kappa_{e x}(x, \theta)$ (possibly discretized in space) defined on the probability space $(\Theta, \mathcal{B}, P)$. The solution $\mathbf{u}_{e x} \in \mathbb{R}^{n} \otimes L^{2}(\Theta, d P)$ of the semi-discretized problem is then defined by

$$
\begin{aligned}
& A_{e x}\left(\mathbf{u}_{e x}, \mathbf{v}\right)=E\left(\mathbf{v}^{T} \mathbf{b}\right) \quad \forall \mathbf{v} \in \mathbb{R}^{n} \otimes L^{2}(\Theta, d P), \\
& \text { with } \quad A_{e x}(\mathbf{u}, \mathbf{v})=E\left(\mathbf{v}^{T} \mathbf{A}_{e x} \mathbf{u}\right) .
\end{aligned}
$$

Let us now introduce a polynomial chaos representation of $\kappa_{e x}$ (see appendix A) and let us consider that the discretized field $\kappa$ is obtained by truncating this representation to the polynomial chaos of degree $2 p$ in dimension $m$ :

$$
\begin{aligned}
\kappa_{e x}(x, \theta) & =\sum_{\alpha \in \mathcal{J}_{e x}} \kappa_{\alpha}(x) H_{\alpha}\left(\left\{\xi_{i}\right\}_{i \in \mathbb{N}}\right) \\
& \approx \sum_{\alpha \in \mathcal{J}_{P_{A}}} \kappa_{\alpha}(x) H_{\alpha}(\boldsymbol{\xi}(\theta)):=\kappa(x, \boldsymbol{\xi}(\theta)),
\end{aligned}
$$

where $\left\{H_{\alpha}\right\}_{\alpha \in \mathcal{J}_{e x}}$ denotes the basis of $L^{2}(\Theta, d P)$ constituted by Hermite polynomial in independent standard Gaussian random variables $\left\{\xi_{i}\right\}_{i \in \mathbb{N}}[130,23]\left(\mathcal{J}_{e x}\right.$ is the set of multi-indices $\alpha \in \mathbb{N}^{\mathbb{N}}$ with finite length $\left.|\alpha|\right)$. Then, one considers for $\mathcal{S}_{P}$ a polynomial chaos of degree $p$ in dimension $m$. From orthogonality of Hermite polynomials, one can show that the restriction to $\mathbb{R}^{n} \otimes \mathcal{S}_{P}$ of bilinear form $A_{e x}(\cdot, \cdot)$ coincides with bilinear form $A(\cdot, \cdot)$, defined from $\kappa$. In other words, the Galerkin approximation $\mathbf{u} \in \mathbb{R}^{n} \otimes \mathcal{S}_{P}$, defined by (51), is the projection of $\mathbf{u}_{e x}$ on $\mathbb{R}^{n} \otimes \mathcal{S}_{P}$ with respect to the inner product induced by $A_{e x}$. Therefore, the Galerkin procedure allows to take implicitly into account the initial non discretized stochastic field. When using discretized versions of stochastic fields, the Galerkin method allows to avoid the classical problem of violation of existence conditions (see remark 6). We can notice that this "good" property may still be verified for some nonlinear problems.

Remark 10 - The particular case of stochastic interpolation/collocation. In the case of a linear problem with a linear dependency of $\mathbf{A}$ in $\boldsymbol{\xi}$, i.e.

$$
\mathbf{A}(\boldsymbol{\xi})=\sum_{\substack{\gamma \in \mathbb{N}^{m} \\|\gamma| \leqslant 1}} \boldsymbol{\xi}^{\gamma} \mathbf{A}_{\gamma}, \quad \boldsymbol{\xi}^{\gamma}:=\prod_{i=1}^{m} \xi_{i}^{\gamma_{i}}, \quad \mathbf{A}_{\gamma} \in \mathbb{R}^{n \times n},
$$

the use of an approximation with Lagrange interpolants (see section 3.2.3) allows obtaining the Galerkin solution $\mathbf{u}$ by a simple "stochastic collocation" method, equivalent to the resolution of $P$ uncoupled deterministic problems. This comes from orthogonality properties (30) and (31) of the basis functions. Denoting by $\left\{\mathbf{y}_{\alpha}\right\}_{\alpha \in \mathcal{J}_{P}}$ the interpolation points associated with a Gaussian quadrature (obtained by tensorization of one-dimensional interpolation grids), and by $\left\{\omega_{\alpha}\right\}_{\alpha \in \mathcal{J}_{P}}$ the weights of the associated quadrature, one has

$$
E\left(\boldsymbol{\xi}^{\gamma} H_{\alpha} H_{\beta}\right)=\mathbf{y}_{\alpha}^{\gamma} \omega_{\alpha} \delta_{\alpha \beta}, \quad \forall \gamma \text { such that }|\gamma| \leqslant 1 .
$$

In this case, system (58) is equivalent to the following $P$ uncoupled systems:

$$
\left(\sum_{\substack{\gamma \in \mathbb{N}^{m} \\|\gamma| \leqslant 1}} \mathbf{y}_{\alpha}^{\gamma} \mathbf{A}_{\gamma}\right) \mathbf{u}_{\alpha} \omega_{\alpha}=E\left(\mathbf{b} H_{\alpha}\right), \quad \alpha \in \mathcal{J}_{P} .
$$

For the example of section 4.1.4, this (very) particular case occurs if the stochastic field writes $\kappa(x, \theta)=\kappa_{0}(x)+\sum_{i=1}^{m} \xi_{i}(\theta) \kappa_{i}(x)$. 
4.4 Classical solution techniques

\subsubsection{Linear problems}

In the linear case, iterative solvers are generally used for solving system (59). Krylovtype solvers (Conjugate Gradient, Conjugate Gradient Square,...) do not require the assembling of matrix A. An efficient preconditioning of the system is however necessary. Krylov-type algorithms are then applied to the following preconditioned system:

$$
\mathbf{P A u}=\mathbf{P b} .
$$

In the case when an orthonormal basis $\left\{H_{\alpha}\right\}$ is used, a classical choice of preconditioner $[44,99,63]$ consists in taking a block diagonal matrix $\mathbf{P}$, the diagonal blocks being defined by

$$
(\mathbf{P})_{\alpha \beta}=\delta_{\alpha \beta} E(\mathbf{A})^{-1}
$$

This preconditioner is computationally very cheap and is relatively efficient in the case where the variability of operator $\mathbf{A}$ is small. Indeed, if matrix $\mathbf{A}$ is decomposed into its mean part $E(\mathbf{A})$ and its centered part, matrix $\mathbf{A}$ of system (64) writes:

$$
(\mathbf{A})_{\alpha \beta}=\delta_{\alpha_{\beta}} E(\mathbf{A})+E\left(H_{\alpha} H_{\beta}(\mathbf{A}-E(\mathbf{A}))\right) .
$$

and tends to $\mathbf{P}^{-1}$ when the variance of $\mathbf{A}$ tends to zero. For large variabilities of the operator, the convergence of Krylov-type algorithms may drastically deteriorate. One can find in [101] a similar construction of the preconditioner in the case of mixed formulations.

\subsubsection{Nonlinear problems}

In the nonlinear context, classical nonlinear solvers may be used for solving system (53). Let us here simply illustrate the use of classical Newton or Quasi-Newton methods [35]. Knowing the iterate $\mathbf{u}^{(k)} \in \mathbb{R}^{n} \otimes \mathcal{S}_{P}$, an increment $\mathbf{w} \in \mathbb{R}^{n} \otimes \mathcal{S}_{P}$ is searched by solving the following linear problem:

$$
A^{(k)}(\mathbf{w}, \mathbf{v})=B(\mathbf{v})-A\left(\mathbf{u}^{(k)}, \mathbf{v}\right) \quad \forall \mathbf{v} \in \mathbb{R}^{n} \otimes \mathcal{S}_{P},
$$

where $A^{(k)}(\cdot, \cdot)$ is an approximation of the bilinear form tangent to $A$ at $\mathbf{u}^{k}$, which can be written under a discrete form:

$$
A^{(k)}(\mathbf{w}, \mathbf{v})=E\left(\mathbf{v}^{T} \mathbf{A}^{(k)} \mathbf{u}\right)
$$

where $\mathbf{A}^{k}$ is a matrix (possibly random) approximating the tangent matrix at $\mathbf{u}^{(k)}$. From an algebraic point of view, this is equivalent to solving system (54) iteratively, by solving at each iteration a linear system of size $n \times P$ of the form

$$
\mathbf{A}^{(k)} \mathbf{w}=\mathbf{r}^{(k)},
$$

where $\mathbf{r}^{(k)}:=\mathbf{b}-\mathbf{A}\left(\mathbf{u}^{(k)}\right)$ is the residual at iteration $k$. Systems (66) can then be solved with the Krylov-type iterative solvers mentioned in section 4.4.1.

In general, the selection of a nonlinear solver for a given application is inspired from classical solvers which are used in the deterministic context. Let us note that a non 
negligible part of the computational times comes from the evaluation of residuals and therefore from the computation of right-hand sides of systems (66). These evaluations may be easy for certain types of nonlinearities (simple form of the nonlinear operator) $[73,74,56]$. In a more general context, projection techniques using adapted stochastic quadratures may be used to perform these evaluations [61,84].

Let us mention some examples of nonlinear solvers. In [84], one can find the application of a BFGS solver in the case of an elliptic diffusion problem with cubic nonlinearity. The advantage is to build $\mathbf{A}^{(k)}$ by simple low rank modification of an initial matrix $\mathbf{A}^{(0)}$ (based for example on the linear part of operator A). Krylov-type preconditioned iterative solvers are then used to solve systems (66). In [90], a classical Newton-type solver is used to solve the same problem. Still for the same problem, it is proposed in [94] to use for $\mathbf{A}^{(k)}$ an approximation of the Gateaux derivative of $\mathbf{A}$ at $\mathbf{u}^{(k)}$. This approximation consists in replacing random parameters of operator $\mathbf{A}$ by their mean values and to replace $\mathbf{u}^{(k)}$ by its mean value. This construction, relatively efficient for moderate variabilities, has the advantage to yield to a deterministic matrix $\mathbf{A}^{(k)}$ and then to a block-diagonal system (66) (resolution of uncoupled deterministic problems).

\subsubsection{Sparse (or not sparse) structure of linear systems}

Krylov-type iterative techniques for the resolution of system (59) (or (66)) only ask for computing matrix-vector products of type Au. This allows to take part of the (possibly) sparse structure of the matrix. The matrix has often a sparse structure at two levels, coming from the possible sparsity of random matrix $\mathbf{A}$ (classical in finite elements, finite difference,...) but also from properties of basis functions $\left\{H_{\alpha}\right\}$. Indeed, the term $E\left(H_{\gamma} H_{\alpha} H_{\beta}\right)$ generally has a sparse structure for classical approximation basis. Figure 10 illustrates the sparsity pattern of matrix $\sum_{\gamma \in \mathcal{J}_{P_{A}}} E\left(H_{\gamma} H_{\alpha} H_{\beta}\right)$, reflecting the blocksparsity pattern of system (59). We can note that the sparse structure strongly depends on the dependence of $\mathbf{A}$ with respect to $\boldsymbol{\xi}$. If a high order is used for the expansion of $\mathbf{A}$ on the basis $\left\{H_{\alpha}\right\}$, we clearly loose the block-sparsity of the system.

\section{Model reduction techniques}

\subsection{Limitations of classical Galerkin spectral stochastic methods}

Galerkin-type spectral stochastic methods have the capability to provide highly accurate numerical predictions. As shown in section 4, they ask for the resolution of a problem which can be formally written: find an approximate solution $u$ such that

$$
u \in \mathcal{V}_{n} \otimes \mathcal{S}_{P}, \quad A(u, v)=B(v) \quad \forall v \in \mathcal{V}_{n} \otimes \mathcal{S}_{P},
$$

where $\mathcal{V}_{n}$ (resp. $\left.\mathcal{S}_{P}\right)$ is a deterministic (resp. stochastic) approximation space of dimension $n$ (resp. $P)^{8}$. For complex applications, if one tries to obtain accurate numerical predictions, these approaches may require a fine discretization at the deterministic level (large $n$ ) or at the stochastic level (large $P$ ). That leads to the resolution of very

\footnotetext{
8 Let us mention that the discretized problem (67) can be interpreted as (51), by assimilating $\mathcal{V}_{n}$ to $\mathbb{R}^{n}$. Then, the discrete formulation (67) is quite general and is also valid for non Galerkin approaches at the deterministic level.
} 


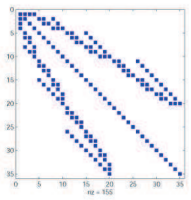

(a) $m=3, p=$ $p_{A}=1$

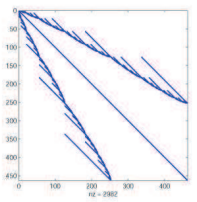

e) $m=5, p=$ $p_{A}=1$

(b) $m$
$p_{A}=2$

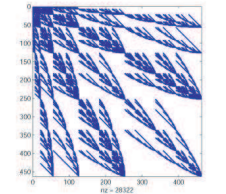

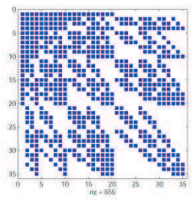

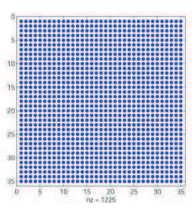

(d) $m=3, p=4$, $p_{A}=3$

$p_{A}=8$
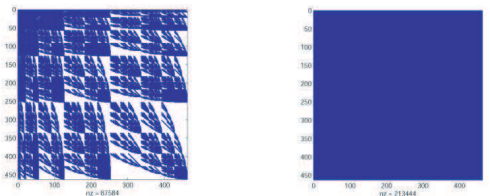

(f) $m=5, p=6$, (g) $m=5, p=6$, (h) $m=5, p=6$ $p_{A}=5$ $p_{A}=12$

Fig. 10 Sparsity pattern of matrix $\sum_{\gamma \in \mathcal{J}_{P_{A}}} E\left(H_{\gamma} H_{\alpha} H_{\beta}\right), \alpha, \beta \in \mathcal{J}_{P}$ : use of a Hermite polynomial chaos in dimension $m$, with a degree $p$ for $\mathcal{J}_{P}$ and a degree $p_{A}$ for $\mathcal{J}_{P_{A}}$

large system of $n \times P$ equations, leading to computational times and memory requirements which are not compatible with available computational resources. Moreover, they require a good knowledge of the mathematical structure of the problem in order to choose a well-adapted discretized formulation (67) (e.g. stabilized formulation when needed), to derive ad hoc efficient solvers or to extend classical deterministic solvers to the stochastic context (nonlinear algorithms, dedicated preconditioners, ...). They often require specific theoretical and software developments for a particular class of problems.

In order to limit computational costs, a first approach consists in judiciously choosing the approximation space $\mathcal{S}_{P}$, leading to an accurate expansion of the solution while minimizing the dimension $P$. In particular, a judicious choice of random variables defining the basic probability space $\left(\boldsymbol{\Xi}, \mathcal{B} \boldsymbol{\Xi}, P_{\boldsymbol{\xi}}\right)$ may allow constructing orthogonal polynomial basis exhibiting good convergence rates [132]. In the case of non-smooth solutions, these convergence rates may deteriorate. They can be improved by using adapted basis such that finite elements, multi-elements or multi-wavelets [34,128, 71, $72,9]$ (see section 3.3). However, a good approximation space can not be chosen without error estimation criteria. This kind of approach then makes sense in the context of adaptive approximation procedures [62, 128, 72, 129,81].

\subsection{Model reduction for Galerkin spectral stochastic methods}

In order to drastically reduce computational costs of Galerkin-type stochastic methods, another approach consists in building reduced approximation basis intelligently. That consists in searching a set of $M$ deterministic functions $w_{i} \in \mathcal{V}$ (or stochastic functions $\lambda_{i} \in \mathcal{S}$ ), with $M \ll n$ (or $M \ll P$ ) and then to compute the associated stochastic functions $\lambda_{i}$ (or deterministic functions $w_{i}$ ). An approximation $u_{M}$ of problem (67) is 
then searched under the form:

$$
u_{M}=\sum_{i=1}^{M} w_{i} \lambda_{i}, \quad w_{i} \in \mathcal{V}, \quad \lambda_{i} \in \mathcal{S} .
$$

On one side, when a reduced basis of deterministic functions is available, it defines an approximation space $\mathcal{V}_{M}=\operatorname{span}\left\{w_{i}\right\}_{i=1}^{M} \subset \mathcal{V}$. Approximation (68) can then be naturally defined by the following Galerkin orthogonality criterium:

$$
\begin{aligned}
& u_{M} \in \mathcal{V}_{M} \otimes \mathcal{S}, \\
& A\left(u_{M}, v_{M}\right)=B\left(u_{M}\right) \quad \forall v_{M} \in \mathcal{V}_{M} \otimes \mathcal{S} .
\end{aligned}
$$

By introducing an approximation space $\mathcal{S}_{P} \subset \mathcal{S}$ in equation (69), it leads to a system of $M \times P$ equations.

On the other side, when a reduced basis of stochastic functions is available, it defines an approximation space $\mathcal{S}_{M}=\operatorname{span}\left\{\lambda_{i}\right\}_{i=1}^{M} \subset \mathcal{S}$. Approximation (68) can still be naturally defined by the following Galerkin orthogonality criterium:

$$
\begin{aligned}
& u_{M} \in \mathcal{V} \otimes \mathcal{S}_{M}, \\
& A\left(u_{M}, v_{M}\right)=B\left(u_{M}\right) \quad \forall v_{M} \in \mathcal{V} \otimes \mathcal{S}_{M} .
\end{aligned}
$$

When introducing an approximation space $\mathcal{V}_{n} \subset \mathcal{V}$ in equation (71), it leads to a system of $n \times M$ equations.

The question is then: how to define reduced basis leading to an optimal decomposition of the solution for a given order $M$ of decomposition?

\subsubsection{Classical spectral decomposition and related techniques}

One way to define optimal basis, explored in different works $[84,47,38]$, is based on the following property: the optimal decomposition of type (68) in the sense of a natural norm $\|\cdot\|$ in $\mathcal{V} \otimes S$ is a classical spectral decomposition of the solution. Optimal basis are defined by:

$$
\left\|u-u_{M}\right\|^{2}=\min _{\substack{\left\{\lambda_{i}\right\}_{i=1}^{M} \in(\mathcal{S})^{M} \\\left\{w_{i}\right\}_{i=1}^{M} \in(\mathcal{V})^{M}}}\left\|u-\sum_{i=1}^{M} w_{i} \lambda_{i}\right\|^{2}
$$

Of course, the obtained decomposition depends on the chosen norm. Let $\langle\cdot, \cdot\rangle_{\mathcal{V}}$ denote an inner product on Hilbert space $\mathcal{V}$, with associated norm $\|\cdot\|_{\mathcal{v}}$. A natural choice consists in introducing for $\|\cdot\|$ the natural norm on $\mathcal{V} \otimes L^{2}\left(\boldsymbol{\Xi}, d P_{\boldsymbol{\xi}}\right)$ :

$$
\|u\|^{2}=E(<u, u>\mathcal{V}):=\int_{\Xi}<u, u>\mathcal{V} d P_{\boldsymbol{\xi}}
$$

For this choice, it is well known that the optimal decomposition defined by (72) is the Hilbert Karhunen-Loève decomposition $[75]^{9}$, truncated at order $M$ (see appendices A

${ }^{9}$ Let us consider that $\mathcal{V}$ is a Hilbert space of functions defined on a domain $\Omega$ (time, space or space-time domain). If $\mathcal{V} \hookrightarrow L^{2}(\Omega)$ and if we choose for $\langle\cdot, \cdot\rangle_{\mathcal{V}}$ the natural inner product in $L^{2}(\Omega)$, the optimal decomposition is the classical Karhunen-Loève decomposition (see appendix A). 
and B). Optimal reduced basis functions span the dominant eigenspace of the following eigenproblem:

$$
T_{u}(w)=\sigma w,
$$

where $T_{u}: \mathcal{V} \rightarrow \mathcal{V}$ is the correlation operator defined by: $\forall w, w^{*} \in \mathcal{V}$,

$$
\begin{aligned}
<w^{*}, T_{u}(w)>_{\mathcal{V}} & =E\left(<w^{*}, u>_{\mathcal{v}}<u, w>_{\mathcal{V}}\right) \\
& :=<w^{*},<E(u \otimes u), w>_{\mathcal{V}}>_{\mathcal{V}},
\end{aligned}
$$

where $E(u \otimes u) \in \mathcal{V} \otimes \mathcal{V}$ is the correlation function of $u$. If we choose the $\left\{w_{i}\right\}_{i=1}^{M}$ such that $\operatorname{span}\left\{w_{i}\right\}_{i=1}^{M}$ is the $M$-dimensional dominant eigenspace of $T_{u}$, and if we define the associated stochastic functions $\left\{\lambda_{i}\right\}_{i=1}^{M}$ such that

$$
\left\|u-\sum_{i=1}^{M} w_{i} \lambda_{i}\right\|^{2}=\underset{\left\{\lambda_{i}\right\}_{i=1}^{M} \in(\mathcal{S})^{M}}{\min }\left\|u-\sum_{i=1}^{M} w_{i} \lambda_{i}\right\|^{2},
$$

we classically show that the obtained decomposition $u_{M}$ verifies:

$$
\left\|u-u_{M}\right\|^{2}=\|u\|^{2}-\sum_{i=1}^{M} \sigma_{i}
$$

where the $\sigma_{i}$ are the $M$ dominant eigenvalues of $T_{u}$.

Therefore, if one could compute the spectral decomposition of the solution, one could consider the stochastic (resp. deterministic) functions of this decomposition as good candidates for the definition of reduced basis of stochastic (resp. deterministic) functions. The problem is that the solution and a fortiori its correlation structure, is not known a priori.

Several techniques have been proposed in order to obtain an approximation of the spectral decomposition. In [84], the authors propose to compute an approximation of the correlation function $E(u \otimes u)$ based on a truncated Neumann expansion of the solution of (67). Dominant eigenfunctions of the corresponding approximation of $T_{u}$ are then computed and can be considered as approximations of functions appearing in the ideal spectral decomposition. Then, they can be used for solving the initial problem in the reduced approximation space $\mathcal{V}_{M} \otimes \mathcal{S}_{P}$ (problem (69)). In its actual form, this procedure is limited to the linear case. In $[47,38]$, the authors propose to first solve the initial problem on a coarse deterministic approximation space $V_{n^{\prime}}$ (e.g. by using a finite element approximation on a coarse mesh). A spectral (Hilbert Karhunen-Loève) decomposition of the coarse solution in $\mathcal{V}_{n^{\prime}} \otimes \mathcal{S}_{P}$ is then performed. After a truncation of the decomposition at order $M$, the obtained random variables $\lambda_{i} \in \mathcal{S}_{P}$ can be considered as an approximation of random variables appearing in the ideal spectral decomposition of the solution in $V_{n} \otimes \mathcal{S}_{P}$. They can then be used as new stochastic basis functions for the resolution of the initial problem in the reduced approximation space $\mathcal{V}_{n} \otimes \mathcal{S}_{M}($ problem $(71))$.

Remark 11 - Equivalent eigenvalue problem.

The spectral decomposition can be equivalently obtained by solving an eigenproblem on $\lambda$ :

$$
T_{u}^{\diamond}(\lambda)=\sigma \lambda,
$$


where $T_{u}^{\diamond}: \mathcal{S} \rightarrow \mathcal{S}$ is defined by: $\forall \lambda, \lambda^{*} \in \mathcal{S}$,

$$
E\left(\lambda^{*} T_{u}^{\diamond}(\lambda)\right)=<E\left(\lambda^{*} u\right), E(u \lambda)>v .
$$

\subsubsection{Generalized spectral decomposition method}

The methods presented in section 5.2.1 can be considered as a posteriori model reduction techniques since they ask for a first evaluation of the solution in order to build the reduced basis.

The Generalized Spectral Decomposition (GSD) method [89-91,94] can be considered as an a priori model reduction technique in the context of Galerkin spectral stochastic methods. The GSD method allows the construction of the decomposition (68) without $a$ priori knowing the solution nor an approximation of it. The basic principle of the GSD method consists in defining optimal reduced basis from a double Galerkin orthogonality criterium. More precisely, it consists in defining reduced approximation spaces $V_{M}$ and $\mathcal{S}_{M}$ such that they verify simultaneously equations (69) and (71). One then shows that reduced basis are solution of an invariant subspace problem. This problem can be assimilated to an eigenproblem whose dominant eigenspace leads to the researched reduced basis functions. The GSD method has been initially introduced for solving a particular class of linear elliptic stochastic partial differential equations [89]. In this context, the method appears as a natural extension of Hilbert Karhunen-Loève decomposition (see appendix B). Dedicated algorithms, inspired from classical algorithms for solving eigenproblems, have been proposed for the construction of reduced basis functions. The main advantage of these algorithms is that they only require the resolution of a few deterministic problems, with a well mastered mathematical structure, and of a few stochastic algebraic equations. Computational costs are then drastically reduced. Moreover, stochastic equations and deterministic problems being uncoupled, the GSD method allows for recovering a part of non intrusivity for Galerkin spectral approaches.

In [90], the method has been used for solving a nonlinear stochastic elliptic problem for which a classical global nonlinear solver led to the resolution of successive linear stochastic problems. Each linear stochastic problem were solved by GSD algorithms proposed in [90], with a re-use and an enrichment of the reduced basis of deterministic functions at each iteration of the nonlinear solver. The GSD method has been extended to a wider class of linear problems in [91], where it has been also proposed some new efficient algorithms for building the generalized decomposition. More recently, a natural extension to the non-linear context has been proposed in [94].

The basics of the method are detailed in section 6 .

Remark 12 - In fact, the GSD method can be seen as a model reduction technique for solving problems defined in tensor product spaces. The GSD method for stochastic problems is inspired from a separated representation technique, called the radial approximation technique, proposed in the context of deterministic space-time problems [68, 70, 93, 3].

5.3 Other model reduction techniques

5.3.1 Non intrusive stochastic approaches and model reduction

Here, we briefly come back to so-called non intrusive stochastic approaches (e.g. Monte Carlo or direct spectral stochastic approaches, ...). These approaches are based on the 
strong formulation (34) at the stochastic level and require the resolution of a huge number of deterministic problems of type: $u\left(\boldsymbol{\xi}_{k}\right) \in \mathcal{V}_{n}$,

$$
a\left(u\left(\boldsymbol{\xi}_{k}\right), v ; \boldsymbol{\xi}_{k}\right)=b\left(v ; \boldsymbol{\xi}_{k}\right), \forall v \in \mathcal{V}_{n}
$$

Their efficiency could be significantly improved by model reduction techniques classically used in parametric or multi-resolution analysis, i.e. by using in (81) a low dimensional approximation space $\mathcal{V}_{M} \subset \mathcal{V}_{n}$, with $M \ll n$. Approximate solutions of problems (81) can then be defined by: $u_{M}\left(\boldsymbol{\xi}_{k}\right) \in \mathcal{V}_{M}$,

$$
a\left(u_{M}\left(\boldsymbol{\xi}_{k}\right), v_{M} ; \boldsymbol{\xi}_{k}\right)=b\left(v_{M} ; \boldsymbol{\xi}_{k}\right), \forall v_{M} \in \mathcal{V}_{M}
$$

Model reduction techniques based on the Proper Orthogonal Decomposition (POD) [131] may be used for constructing a reduced approximation space $V_{M}$. It consists in solving several fine deterministic problems (81), leading to a collection of functions $u\left(\boldsymbol{\xi}_{k}\right) \in \mathcal{V}_{n}$. Then, a proper orthogonal decomposition (or singular value decomposition, or discrete Karhunen-Loève decomposition) of this collection of functions allows to capture the $M$ most significant modes $\left\{w_{i}\right\}_{i=1}^{M}$, thus defining $\mathcal{V}_{M}=\operatorname{span}\left\{w_{i}\right\}_{i=1}^{M}$. Error criteria must clearly be provided in order to estimate the error associated with $u_{M}$ and to eventually enrich the approximation space $\mathcal{V}_{M}$.

An alternative model reduction technique has been proposed in the context of multiresolution analysis and Krylov-type iterative solvers [111]. When a problem (81) is solved with a Krylov iterative algorithm, the generated Krylov subspace defines a lowdimensional approximation space $\mathcal{V}_{M}$ leading to an accurate solution for this particular problem. This subspace may be efficiently re-used for subsequent deterministic problems (81) [110,106,51]. First, it can be used for computing an initial approximation by solving the reduced problem (82). Then, starting from an updated residual, Krylov algorithms can be used (with eventual projections in order to avoid exploring again the initial subspace $\left.\mathcal{V}_{M}\right)$. The reduced approximation space $\mathcal{V}_{M}$ can be updated after each resolution. A difficult question concerns the selection of pertinent subspaces of $\mathcal{V}_{M}$ for the subsequent resolutions, in order to avoid a dramatic increase in the dimension of $v_{M}$.

Let us finally mention another model reduction technique, called the "Reduced Basis" method [79,80,11], which has been initially introduced for parametric analysis. This method, based on rigorous error estimation criteria, proposes a construction of approximation space $\mathcal{V}_{M}$ leading to a desired accuracy for $u_{M}\left(\boldsymbol{\xi}_{k}\right)$ for all $k$. The advantage is that it never requires the resolution of fine problems (81). However, the method requires theoretical developments which are specific to the considered problem. The "Reduced Basis" method has been recently applied to the resolution of linear stochastic elliptic symmetric problems with the Monte-Carlo method [108].

\subsubsection{Stochastic Reduced Basis Method}

Let us mention and give some comments on the Stochastic Reduced Basis Method [86, $87,113,112$ ], proposed for solving a class of linear stochastic problems. The first point of the method consists in computing a basis of functions $w_{i} \in \mathcal{V}_{n} \otimes \mathcal{S}$ by successive applications of the operator to the right-hand side of problem (67), thus generating a so-called "stochastic Krylov subspace". This approach is different from the model reduction techniques mentioned in section 5.2 since functions $w_{i}$ are not deterministic 
but belong to $\mathcal{V}_{n} \otimes \mathcal{S}$. Then, this does not circumvent the problem of memory requirements. In fact, if a classical spectral representation is used at the stochastic level (i.e. successive projections of the $w_{i}$ on $\mathcal{V}_{n} \otimes \mathcal{S}_{P}$ ), as proposed in [112,113], the defined stochastic Krylov subspace $\operatorname{span}\left\{w_{i}\right\}_{i=1}^{M}$ is no more than a classical $M$-dimensional Krylov subspace associated with problem (67). To be more precise, the defined Krylov subspace is exactly the Krylov subspace of the preconditioned system (64), with the classical preconditioner used in [46,44,63]. Indeed, if we rewrite problem (67) under the form of the linear system of equations (59), the definition of functions $w_{i} \sim \mathbf{w}_{i} \in \mathbb{R}^{n P}$ given in $[112,113]$ is as follows: $\mathbf{w}_{i}=(\mathbf{P A})^{i-1} \mathbf{b}, i=1 \ldots M$.

In [113], the authors then propose to define the approximation $u_{M}=\sum_{i=1}^{M} w_{i} \lambda_{i}$, with $\lambda_{i} \in \mathbb{R}$, where the $\lambda_{i}$ are solutions of the following system of $M$ equations:

$$
A\left(\sum_{i=1}^{M} w_{i} \lambda_{i}, w_{j}\right)=B\left(w_{j}\right), \quad \forall j \in\{1 \ldots M\} .
$$

The obtained solution $u_{M}$ is the Galerkin approximation of the initial problem in the approximation space $\operatorname{span}\left\{w_{i}\right\}_{i=1}^{M} \subset \mathcal{V}_{n} \otimes \mathcal{S}_{P}$. In fact, system (83) is equivalent to the following system, written in a matrix form:

$$
\mathbf{w}_{j}^{T} \mathbf{A} \sum_{i=1}^{M} \mathbf{w}_{i} \lambda_{i}=\mathbf{w}_{j}^{T} \mathbf{b}, \quad \forall j \in\{1 \ldots M\} .
$$

The method proposed in [113] then exactly coincides with a classical Krylov-type algorithm, namely the Arnoldi algorithm, for solving (59) (equivalently (67)). Therefore, this method does not really constitute a new methodology and can not really be assimilated with a model reduction technique, although Krylov-type iterative solvers are sometimes seen as a posteriori model reduction techniques. In [113], the authors conclude that a low order approximation $(M=2$ or 3$)$ is sufficient for obtaining an accurate solution. It is equivalent to say that a Krylov-type algorithm (e.g. Preconditioned Conjugate Gradient for symmetric problems) gives an accurate solution of (59) in 2 or 3 iterations. That is clearly problem-dependent and it is known that for complex problems and moderate variabilities, Krylov-type algorithms may require a much larger number of iterations in order to provide an accurate approximation.

However, a modification is proposed in another paper [112]. Starting from the above defined reduced basis of functions $\left\{w_{i}\right\}_{i=1}^{M} \in\left(\mathcal{V}_{n} \otimes \mathcal{S}_{P}\right)^{M}$, the authors propose to search an approximation $u_{M}=\sum_{i=1}^{M} w_{i} \lambda_{i}$, with random functions $\lambda_{i} \in \mathcal{S}_{P}$ defined by the following problem:

$$
A\left(\sum_{i=1}^{M} w_{i} \lambda_{i}, \sum_{i=1}^{M} w_{i} \lambda_{i}^{*}\right)=B\left(\sum_{i=1}^{M} w_{i} \lambda_{i}^{*}\right) \quad \forall \lambda_{i}^{*} \in \mathcal{S}_{P} .
$$

In this case, the method can not be interpreted as a usual Krylov-type algorithm. This modification slightly improves the accuracy of the obtained decomposition. Let us note that $u_{M}$ is a nonconforming Galerkin approximation since $u_{M} \notin \mathcal{V}_{n} \otimes \mathcal{S}_{P}$. The construction and the resolution of problem (85) for finding functions $\lambda_{i}$ are difficult and computationally expansive (more expansive than classical Galerkin approaches since the $w_{i}$ are random), which leads to limit the number of computed functions $w_{i}$. 
6 Generalized spectral decomposition method

In this section, we recall the basics of the GSD method [89-91,94], mentioned in section 5.2 .2 , and give some additional and clarifying comments on the method.

6.1 Definition of the generalized spectral decomposition

A natural way to define optimal reduced basis is to use a double orthogonality criterium, i.e. to verify both equations $(71)$ and (69) simultaneously. Let us note $\Lambda=\left(\lambda_{i}\right)_{i=1}^{M} \in$ $(\mathcal{S})^{M}, W=\left(w_{i}\right)_{i=1}^{M} \in(\mathcal{V})^{M}$ and $u_{M}:=W \cdot \Lambda$. Equations (71) and (69) can be equivalently rewritten:

$$
\begin{array}{ll}
A\left(W \cdot \Lambda, W^{*} \cdot \Lambda\right)=B\left(W^{*} \cdot \Lambda\right) & \forall W^{*} \in(\mathcal{V})^{M}, \\
A\left(W \cdot \Lambda, W \cdot \Lambda^{*}\right)=B\left(W \cdot \Lambda^{*}\right) & \forall \Lambda^{*} \in(\mathcal{S})^{M} .
\end{array}
$$

Let us introduce the mapping $F:(\mathcal{S})^{M} \rightarrow(\mathcal{V})^{M}$ where $W=F(\Lambda)$ is the solution of equation (86) for a given $\Lambda$. Let us also introduce the mapping $f:(\mathcal{V})^{M} \rightarrow(\mathcal{S})^{M}$ where $\Lambda=f(W)$ is the solution of equation (87) for a given $W$. The simultaneous verification of (87) and (86) imposes the following relations:

$$
W=F(\Lambda) \quad \text { and } \quad \Lambda=f(W) .
$$

Equations (88) can be interpreted as a problem on $W$ :

$$
T(W)=W, \quad \text { with } \quad T(W)=F \circ f(W),
$$

or equivalently as a problem on $\Lambda$ :

$$
T^{\diamond}(\Lambda)=\Lambda, \quad \text { with } \quad T^{\diamond}(\Lambda)=f \circ F(\Lambda) .
$$

From homogeneity properties of $T$ and $T^{\diamond}$, problems (89) and (90) can be interpreted as invariant subspace problem, i.e. they can be equivalently written as fixed point problems on $\mathcal{V}_{M}$ and $\mathcal{S}_{M}$ (see [91]). In fact, these problems can be interpreted as eigenlike problems, where an invariant subspace is assimilated with a generalized eigenspace. The dominant eigenspace of $T$ (resp. $T^{\diamond}$ ) is associated with the researched reduced basis $W\left(\right.$ resp. $\Lambda$ ), which leads to a so-called generalized spectral decomposition $u_{M}=W$. $f(W)$ (resp. $\left.u_{M}=F(\Lambda) \cdot \Lambda\right)$. The method can be interpreted as a natural generalization of Hilbert Karhunen-Loève decomposition (see appendix B for a comprehensive analysis in the case of linear symmetric elliptic problems).

6.2 Algorithms for building the decomposition

Algorithms for building the generalized spectral decomposition are inspired from classical methods for solving eigenproblems $[109,50]$ (for capturing the upper spectrum of operators). 
Problems (88) and (90) being equivalent, let us focus on the problem on $T$. The aim is to capture the dominant eigenspace of $T$, leading to the desired reduced basis of functions $W=\left(w_{i}\right)_{i=1}^{M}$. A natural algorithm, proposed in [91], is the subspace iteration technique. Starting from an initial guess $W^{(0)}$, it consists in building the sequence $W^{(k+1)}=T\left(W^{(k)}\right)$. This sequence generally converges toward the dominant eigenspace of $T$. At each iteration, it requires the application of operator $T=F \circ f$, and therefore, the application of operators $f$ and $F$ successively ${ }^{10}$. The computation of $\Lambda=f(W)$ requires the resolution of problem (87), which is a stochastic problem on a reduced deterministic basis (system of $M$ stochastic algebraic equations, eventually nonlinear). An approximation $\Lambda \in\left(\mathcal{S}_{P}\right)^{M}$ can be obtained by using a Galerkin stochastic approximation, thus requiring the resolution of a system of $M \times P$ equations. Then, the computation of $W=f(\Lambda)$ requires the resolution of problem (86), which is a deterministic problem on a reduced stochastic basis (system of $M$ coupled deterministic problems, eventually nonlinear). An approximation $W \in\left(\mathcal{V}_{n}\right)^{M}$ can be obtained by using a deterministic approximation technique, thus requiring the resolution of a system of $n \times P$ equations. At convergence, we obtained the desired generalized spectral decomposition $u_{M}=W \cdot f(W)$.

\subsubsection{Power method with deflation}

In order to avoid the resolution of coupled systems of deterministic equations, an alternative consists in building the couples $\left(\lambda_{i}, w_{i}\right)$ one by one. Let $u_{r}=W_{r} \cdot \Lambda_{r}$ be an already computed decomposition of order $r$. The following optimal couple $(\lambda, w) \in \mathcal{S}$ can be defined by:

$$
\begin{array}{ll}
A\left(u_{r}+w \lambda, w^{*} \lambda\right)=B\left(w^{*} \lambda\right) & \forall w^{*} \in \mathcal{V}, \\
A\left(u_{r}+w \lambda, w \lambda^{*}\right)=B\left(w \lambda^{*}\right) & \forall \lambda^{*} \in \mathcal{S} .
\end{array}
$$

The problem can still be interpreted as an eigen-like problem

$$
T\left(w ; u_{r}\right)=w, \quad \text { with } T\left(\cdot ; u_{r}\right)=F\left(f\left(\cdot ; u_{r}\right) ; u_{r}\right),
$$

where $\lambda=f\left(w ; u_{r}\right)$ is the solution of problem (92) for a fixed $w$ and where $w=F\left(\lambda ; u_{r}\right)$ is the solution of problem (91) for a fixed $\lambda$.

A natural power method, first introduced in [89], can then be applied in order to find the dominant eigenfunction $w$ of $T\left(\cdot ; u_{r}\right)$. From an initial guess $w^{(0)}$, it consists in building the sequence $w^{(k+1)}=T\left(w^{(k)} ; u_{r}\right)$. At each iteration, it requires the resolution of a simple stochastic algebraic equation (92) (application of operator $f$ for a fixed $w$ ) and of a simple deterministic problem (91) (application of mapping $F$ for a given $\lambda$ ). In fact, operator $T\left(\cdot ; u_{r}\right)$ can be interpreted as a deflated version of the initial operator $T \equiv T(\cdot ; 0)($ see $[91])$.

Remark 13 - Let us note that this algorithm, which is here interpreted as a power method with deflation, have been proposed in other contexts in order to find separated representations of solutions in tensor product spaces (see e.g. [68,70,93,3] for spacetime separation, [27] for space-space separation, [2] for multi-dimensional separation).

\footnotetext{
10 In practise, an orthogonalization step is introduced at each iteration in order to avoid a degeneration of the subspace $\mathcal{V}_{M}:=\operatorname{span}(W)$.
} 
In fact, for the case of elliptic symmetric problems (see appendix B), this algorithm appears as a particular case of a Greedy algorithm [20].

The overall construction can then be interpreted as a power method with deflation for classical eigenproblems. However, eigen-like problem (93) does not have classical properties of eigenproblems. In particular, the $M$-dimensional subspace which is spanned by the dominant eigenfunctions of successive deflated operators $T\left(\cdot ; u_{r}\right), r=$ $0 \ldots M-1$, does not generally coincide with the $M$-dimensional dominant eigenspace of the initial operator $T(\cdot ; 0)$. In other words, the spectral decomposition $u_{M}=W \cdot f(W)$, where $W$ is the dominant eigenspace of $T(\cdot ; 0)$, does not coincide with the spectral decomposition $u_{M}=\sum_{i=1}^{M} w_{i} f\left(w_{i} ; u_{i-1}\right)$, where the $w_{i}$ are the dominant eigenfunctions of $T\left(\cdot ; u_{i-1}\right)$ (see related comments in appendix B). In practise, a better accuracy is obtained by updating the random variables, i.e. by computing a new $\Lambda=f(W ; 0)$ after the construction of $W$ by the power method with deflation (see illustrations [89,94] for linear or nonlinear model problems).

\subsubsection{Arnoldi algorithm}

A more efficient algorithm has been proposed in [89]. It is inspired from the Arnoldi algorithm for solving classical eigenproblems. Starting from an initial function $w_{1}=$ $f\left(\lambda_{1}\right)$, the idea is to build the "generalized Krylov subspace" $\mathcal{K}_{M}\left(T, w_{1}\right)$, defined by:

$$
\begin{aligned}
& \mathcal{K}_{M}\left(T, w_{1}\right)=\operatorname{span}\left\{w_{i}\right\}_{i=1}^{M} \\
& w_{i+1}=\Pi_{\mathcal{K}_{i}}^{\perp} T\left(w_{i}\right),
\end{aligned}
$$

where $\Pi_{\mathcal{K}_{i}}^{\perp}$ denotes an orthogonal projection on the complementary space of $\mathcal{K}_{i} \subset \mathcal{V}$. The construction of the Krylov subspace asks for $M-1$ applications of operator $T=F$ 。 $f$ to single functions in $\mathcal{V}$. It then asks for the resolution of $M-1$ classical deterministic problems (application of $F$ ) and $M-1$ stochastic algebraic equation (application of $f$ ). Then, associated random variables $\Lambda=f(W)$ can be computed by solving a stochastic problem (87) for the given reduced approximation basis $W=\left(w_{i}\right)_{i=1}^{M}$. We observe that this construction allows to obtain at a very low cost a good approximation of the dominant eigenspace, thus leading to a good approximation of the ideal generalized spectral decomposition. In practise, restarts of the above Arnoldi algorithm are often required. When the Arnoldi procedure has stopped, and an order $r$ decomposition $u_{r}$ has been obtained, an Arnoldi procedure can be performed again on the deflated eigenproblem (93) (see [91]).

Remark 14 - Choice of the orthogonal projection.

For classical eigenproblems, this algorithm exactly coincides with a classical Arnoldi algorithm. In this case, the Krylov subspace does not depend on the orthogonal projector. In the case of the GSD, which corresponds to a non classical eigenproblem, the obtained subspace slightly depends on the projection which is used. However, in practise, classical metrics are used for the projections and lead to satisfactory results.

6.3 Illustrations of computational aspects

Here we detail the computational aspects on two model examples in order to illustrate the application of the GSD method. The method is illustrated in a continuous 
framework, the discretization being introduced when needed. It allows to underline an interesting aspect of the GSD method, which provides a flexibility in the choice of deterministic approximation techniques (in a non intrusive fashion). The construction of the generalized spectral decomposition asks for the resolution of problems of different types which depend on the chosen algorithms (power method, Arnoldi,...). Power-type algorithm and Arnoldi algorithm, when applied to eige-like problem $T(W)=W$, asks for the resolution of problems $\lambda=f(w)$ for a given $w \in \mathcal{V}, w=F(\lambda)$ for a given $\lambda \in \mathcal{S}$ and $\Lambda=f(W)$ for a given $W \in(\mathcal{V})^{M}$. For simplicity, only these three types of problems will be detailed for the two model examples.

\subsubsection{Model example 1: a linear problem}

We here consider a linear time-dependent stochastic partial differential equation, defined on a space-time domain $\Omega \times(0, T)$. This class of problem has been investigated in [91]. Computational aspects of the GSD method are here presented in a continuous framework. The discrete formulation and numerical illustrations (convergence of generalized decomposition, efficiency of the method,...) can be found in [91].

We consider the following problem: find $u$ such that it verifies almost surely

$$
\begin{aligned}
& \partial_{t} u-\alpha_{1} \Delta u+\alpha_{2} c \cdot \nabla u=g \text { on } \Omega \times(0, T), \\
& u=0 \text { on } \partial \Omega \times(0, T), \\
& u=0 \text { on } \Omega \times\{0\},
\end{aligned}
$$

where the $\alpha_{i}(\boldsymbol{\xi})$ are random variables defined on probability space $\left(\boldsymbol{\Xi}, \mathcal{B} \boldsymbol{\Xi}, P_{\boldsymbol{\xi}}\right)$ and $g(\boldsymbol{\xi})$ is a random process (time or space dependent). Here, we introduce for $\mathcal{V}$ a suitable space of functions defined on the space-time domain $\Omega \times(0, T)$. A weak formulation can be obtained by introducing bilinear form $A(u, v)=E(a(u, v ; \boldsymbol{\xi}))$ and linear form $B(v)=E(b(v ; \boldsymbol{\xi}))$, where $a$ and $b$ are defined by:

$$
\begin{aligned}
& b(v ; \boldsymbol{\xi})=\int_{\Omega \times(0, T)} v g(\boldsymbol{\xi}), \\
& a(u, v ; \boldsymbol{\xi})=a_{0}(u, v)+\alpha_{1}(\boldsymbol{\xi}) a_{1}(u, v)+\alpha_{2}(\boldsymbol{\xi}) a_{2}(u, v),
\end{aligned}
$$

where the $a_{i}$ are deterministic bilinear forms on $\mathcal{V}$ defined by ${ }^{11}$ :

$$
\begin{aligned}
& a_{0}(u, v)=\int_{\Omega \times(0, T)} \partial_{t} u v+\int_{\Omega \times\left\{0^{+}\right\}} u v, \\
& a_{1}(u, v)=\int_{\Omega} \nabla u \cdot \nabla v \\
& a_{2}(u, v)=\int_{\Omega} c \cdot \nabla u v .
\end{aligned}
$$

\footnotetext{
11 The values of functions on $\Omega \times\left\{0^{+}\right\}$must be interpreted as their right limits at time 0 . The formulation is classical and corresponds to a weak imposition of the initial condition.
} 
$\diamond$ Problem of type $w=F(\lambda)$

Computing $w=F(\lambda)$ for a given $\lambda$ asks for the resolution of a deterministic problem: find $w \in \mathcal{V}$ such that $\forall w^{*} \in \mathcal{V}$,

$$
a_{0}\left(w, w^{*}\right)+\widetilde{\alpha}_{1} a_{1}\left(w, w^{*}\right)+\widetilde{\alpha}_{2} a_{2}\left(w, w^{*}\right)=\int_{\Omega \times(0, T)} \tilde{g} w^{*}
$$

with deterministic parameters $\widetilde{\alpha}_{i}=E\left(\alpha_{i} \lambda^{2}\right)$ and deterministic source term $\widetilde{g}=E(g \lambda)$. Classical deterministic approximation techniques can be used for obtaining an approximate solution $w \in \mathcal{V}_{n}$ (time integration scheme, finite elements, ...). Let us note that suitable time integration schemes (eventually adaptive) can be used for each deterministic independently. This constitutes a great advantage of the generalized spectral decomposition, which authorizes the use of well-mastered deterministic solution techniques.

Remark 15 - In the current version of the GSD method, problems on reduced basis are defined with classical Galerkin projections. For advection-dominated problems, if the deterministic approximation requires a stabilization (e.g. finite elements), the GSD method can still be applied on a stabilized formulation of the initial stochastic problem.

$\diamond$ Problem of type $\lambda=f(w)$

Computing $\lambda=f(w)$ for a given $w$ requires the resolution of:

$$
\lambda \in \mathcal{S}, \quad E\left(\lambda^{*} \widehat{a} \lambda\right)=E\left(\lambda^{*} \widehat{b}\right) \quad \forall \lambda^{*} \in \mathcal{S},
$$

with

$$
\begin{aligned}
& \widehat{b}(\boldsymbol{\xi})=b(w ; \boldsymbol{\xi})=\int_{\Omega \times(0, T)} g(\boldsymbol{\xi}) w \\
& \widehat{a}(\boldsymbol{\xi})=a_{0}(w, w)+\alpha_{1}(\boldsymbol{\xi}) a_{1}(w, w)+\alpha_{2}(\boldsymbol{\xi}) a_{2}(w, w) .
\end{aligned}
$$

This is a classical stochastic algebraic equation. Computing random variables $\widehat{a}$ and $\widehat{b}$ requires computing classical space-time integrals. A classical stochastic Galerkin approach can be used in order to find an approximate solution $\lambda \in \mathcal{S}_{P} \subset \mathcal{S}$. That leads to a small system of $P$ equations.

Remark 16 - When using a deflation (e.g. with a power method or a restarted Arnoldi algorithm), one has to solve problems of type $\lambda=f\left(w ; u_{r}\right)$ or $w=F\left(\lambda ; u_{r}\right)$. These problems simply write as the above problems, with updated right-hand sides $b(v ; \boldsymbol{\xi}) \leftarrow$ $b(v ; \boldsymbol{\xi})-a\left(u_{r}, v ; \boldsymbol{\xi}\right)$.

$\diamond$ Problem of type $\Lambda=f(W)$

Computing $\Lambda=f(W) \in(\mathcal{S})^{M}$ for a given $W=\left(w_{i}\right)_{i=1}^{M} \in(\mathcal{V})^{M}$ requires the resolution of the following system of stochastic algebraic equations:

$$
\sum_{i=1}^{M} E\left(\lambda_{j}^{*} \widehat{a}_{j i} \lambda_{i}\right)=E\left(\lambda_{j}^{*} \widehat{b}_{j}\right) \quad \forall \lambda_{j}^{*} \in \mathcal{S}, \forall j \in\{1, \ldots, M\},
$$

with

$$
\begin{aligned}
& \widehat{b}_{j}(\boldsymbol{\xi})=b\left(w_{j} ; \boldsymbol{\xi}\right)=\int_{\Omega \times(0, T)} g(\boldsymbol{\xi}) w_{j} \\
& \widehat{a}_{j i}(\boldsymbol{\xi})=a_{0}\left(w_{i}, w_{j}\right)+\alpha_{1}(\boldsymbol{\xi}) a_{1}\left(w_{i}, w_{j}\right)+\alpha_{2}(\boldsymbol{\xi}) a_{2}\left(w_{i}, w_{j}\right) .
\end{aligned}
$$


By introducing an approximation space $\mathcal{S}_{P} \subset \mathcal{S}$, we obtain a Galerkin approximate solution $\Lambda \in\left(\mathcal{S}_{P}\right)^{M}$ by solving a classical system of $M \times P$ equations.

Remark 17 - A straightforward extension of the above computational aspects allows dealing with a large class of linear problems such that $a(u, v ; \boldsymbol{\xi})=\sum_{i=1}^{m} \alpha_{i}(\boldsymbol{\xi}) a_{i}(u, v)$, where the $a_{i}$ are deterministic bilinear forms.

\subsubsection{Model example 2: a non-linear problem}

We here consider a classical nonlinear stochastic stationary partial differential equation, with quadratic nonlinearity, defined on a spatial domain $\Omega$ (e.g. stationary Burgers or incompressible Navier-Stokes equations). The application to a one-dimensional stochastic Burgers equation and numerical illustrations can be found in [94].

We introduce a semi-linear form $A(u, v)=E(a(u, v ; \boldsymbol{\xi}))$ and a linear form $B(v)=$ $E(b(v ; \boldsymbol{\xi}))$, where $a$ and $b$ are defined by:

$$
\begin{aligned}
& b(v ; \boldsymbol{\xi})=\int_{\Omega} v g(\boldsymbol{\xi}), \\
& a(u, v ; \boldsymbol{\xi})=\alpha_{1}(\boldsymbol{\xi}) a_{1}(u, v)+a_{2}(u, u, v),
\end{aligned}
$$

where $\alpha_{1}$ and $g$ are respectively a random variable and a random field defined on probability space $\left(\boldsymbol{\Xi}, \mathcal{B} \boldsymbol{\Xi}, P_{\boldsymbol{\xi}}\right) . a_{1}$ and $a_{2}$ are bilinear and trilinear forms defined by:

$$
\begin{aligned}
& a_{1}(u, v)=\int_{\Omega} \nabla u \cdot \nabla v, \\
& a_{2}(u, u, v)=\int_{\Omega} u \cdot \nabla u v .
\end{aligned}
$$

$\diamond$ Problem of type $w=F(\lambda)$

Computing $w=F(\lambda)$ for a given $\lambda$ asks for the resolution of a classical nonlinear deterministic problem: find $w \in \mathcal{V}$ such that $\forall w^{*} \in \mathcal{V}$,

$$
\widetilde{\alpha}_{1} a_{1}\left(w, w^{*}\right)+\widetilde{\alpha}_{2} a_{2}\left(w, w, w^{*}\right)=\int_{\Omega} \widetilde{g} w^{*},
$$

with deterministic parameters $\widetilde{\alpha}_{1}=E\left(\alpha_{1} \lambda^{2}\right)$ and $\widetilde{\alpha}_{2}=E\left(\lambda^{3}\right)$, and deterministic source term $\widetilde{g}=E(g \lambda)$. The method authorizes the use of classical deterministic solvers. In this sense, the GSD method can be said non intrusive. Classical deterministic approximation techniques can be used for obtaining an approximate solution $w \in \mathcal{V}_{n}$. Let us note that for each deterministic problem, a specific solver can be chosen, adapted to the values $\widetilde{\alpha}_{i}$ of parameters.

$\diamond$ Problem of type $\lambda=f(w)$

Computing $\lambda=f(w)$ for a given $w$ requires the resolution of:

$$
\lambda \in \mathcal{S}, \quad E\left(\lambda^{*}\left(\widehat{a}^{(1)} \lambda+\widehat{a}^{(2)} \lambda^{2}\right)\right)=E\left(\lambda^{*} \widehat{b}\right) \quad \forall \lambda^{*} \in \mathcal{S},
$$

with $\widehat{b}(\boldsymbol{\xi})=\int_{\Omega} g(\boldsymbol{\xi}) w, \widehat{a}^{(1)}(\boldsymbol{\xi})=\alpha_{1}(\boldsymbol{\xi}) a_{1}(w, w)$ and $\widehat{a}^{(2)}=a_{2}(w, w, w)$. This is a classical stochastic nonlinear algebraic equation (with quadratic nonlinearity). A classical stochastic Galerkin approach can be used in order to find an approximate solution $\lambda \in \mathcal{S}_{P} \subset \mathcal{S}$. That leads to a small system of $P$ nonlinear equations. 
$\diamond$ Problem of type $\Lambda=f(W)$

Computing $\Lambda=f(W) \in(\mathcal{S})^{M}$ for a given $W=\left(w_{i}\right)_{i=1}^{M} \in(\mathcal{V})^{M}$ requires the resolution of the following system of stochastic algebraic equations: $\forall j \in\{1, \ldots, M\}, \forall \lambda_{j}^{*} \in \mathcal{S}$,

$$
E\left(\lambda_{j}^{*}\left(\sum_{i=1}^{M} \widehat{a}_{j i}^{(1)} \lambda_{i}+\sum_{i, k=1}^{M} \widehat{a}_{j i k}^{(2)} \lambda_{i} \lambda_{k}\right)\right)=E\left(\lambda_{j}^{*} \widehat{b}_{j}\right),
$$

with $\widehat{b}_{j}(\boldsymbol{\xi})=\int_{\Omega} g(\boldsymbol{\xi}) w_{j}, \widehat{a}_{j i}^{(1)}(\boldsymbol{\xi})=a_{1}\left(w_{i}, w_{j}\right)$ and $\widehat{a}_{j i k}^{(2)}=a_{2}\left(w_{i}, w_{k}, w_{j}\right)$. By introducing an approximation space $\mathcal{S}_{P} \subset \mathcal{S}$, we obtain a Galerkin approximate solution $\Lambda \in\left(\mathcal{S}_{P}\right)^{M}$ by solving a system of $M \times P$ nonlinear equations.

\subsubsection{About non intrusivity}

As shown in the two above model examples, the GSD method allows to separate the resolution of classical deterministic problems and stochastic algebraic equations. Deterministic problems may be solved in a non intrusive fashion. Then, Galerkin spectral approaches, when using the GSD method, recover a part of non intrusivity.

The convergence of the generalized spectral decomposition is very similar to the convergence of classical Hilbert Karhunen-Loève decompositions of the solution. For a desired accuracy, the required order of decomposition is clearly problem-dependent. However, in practice, one observes that a low order $(M<10)$ is often sufficient to reach satisfactory accuracies (see illustrations in $[89,91,94]$ ). In these situations, the GSD method leads to accurate predictions by solving a very few deterministic problems, compared to classical non intrusive approaches ( $L^{2}$ projection, Monte-Carlo...).

\section{Conclusion}

Uncertainty quantification appears today as a crucial point in numerous branches of science and engineering. By improving the predictability and robustness of numerical models, it answers technical, economic or societal issues.

In the last two decades, a growing interest has been devoted to a new family of methods, called spectral stochastic approaches, which rely on a fruitful marriage of probability theory and functional analysis. This marriage has allowed to transpose into the stochastic context a set of mathematical and numerical tools which are well mastered within the deterministic context. These methods offer a unified framework for the quantification and propagation of uncertainties in a probabilistic context. In other words, they allow handling both the validation and verification of physical models, from inputs identification based on observations to the numerical prediction of outputs, using a unique formalism.

Although these methods are relatively mature for some classes of problems, numerous theoretical, methodological and technical difficulties still remain to surmount in order to guarantee their long-lasting transfer towards a wider field of applications. First of all, these methods require to revisit in depth fields of applications which are well mastered in the deterministic context. For example, in the field of nonlinear structural dynamics, the current deterministic methods allow to tackle with large-scale simulations while taking into account complex nonlinear behaviors (visco-plasticity, contact, 
damage,...). The treatment of these problems in an uncertain context remains a crucial issue in the medium or long terms. A challenge consists in proposing generic methods, applicable to a wide class of problems and exploiting at best the existing know-how and softwares. The transfer of these methods towards complex industrial applications will be made possible if consequent efforts are led on the development of robust solution techniques, allowing to obtain of predictions with reasonable computation times.

Another key question concerns the control and the reduction of the errors introduced in the various steps of the validation/verification chain: measurement error, modeling error, approximation error, resolution error. Indeed, mastering these various errors would allow to obtain more reliable predictions of the outputs of the models. In an uncertain context, the reduction of measurement errors generally requires to increase the number of observations (if possible) as well as the quality of their statistical treatment. Modeling errors can be reduced through a better understanding of the physical phenomena and sources of uncertainties, and through the development of robust identification procedures in a probabilistic context. The control of approximation requires the development of robust a posteriori error estimators and associated adaptive approximation procedures, in order to improve the quality of the numerical models. Finally, the reduction of resolution errors asks for a better control of the solvers in terms of precision and robustness. These questions of error controlling is certainly an important axis of development for the spectral stochastic approaches. These approaches have the advantage to inherit from an existing know-how in deterministic simulations and in other branches of probabilistic analysis.

Another difficulty, which is not restricted to spectral approaches but to uncertainty quantification methods in general, concerns the relevant modeling of uncertainties. In the physical models, sources of uncertainty appear at various scales, involving different physical phenomena. Uncertainties are often well understood and modeled in certain scales which do not correspond to the scales under study. Fully multi-scale computational methods, allowing to transfer the uncertainties through the scales, could lead to the construction of more relevant models, to more reliable predictions, as well as to a better understanding of the physical phenomena. Beyond these issues, these multiscale strategies will allow to increase the efficiency of the spectral stochastic approaches and thus to solve problems which are currently unaffordable with available computational resources. In this context, the development of model reduction techniques, which is already a challenging issue in the deterministic context, seem to be unavoidable as an additional probabilistic dimension has to be dealt with.

All these questions constitute some important scientific and technical challenges, which could lead to a better control of models and numerical simulations in an uncertain context. It thus remains to hope that future developments will allow to reach a full maturity of these methods and allow their massive use for the resolution of problems of interest.

\section{A Representation and discretization of random processes or fields}

Here, we briefly introduce some classical techniques for representing random processes (or fields). For an introduction to random processes, the reader can refer to $[37,67,1,77,78]$ for a mathematical point of view or to $[98,52,53,126,30]$ for more technical aspects. 
A.1 Definition and characterization of random processes

A random process with value in $\mathcal{E}$ is formally defined as a indexed set of random variables $\left.\left\{u_{x}(\theta)\right)\right\}_{x \in \Omega}$, where the $u_{x}(\theta)$ are random variables with values in $\mathcal{E}$, defined on a probability space $(\Theta, \mathcal{B}, P) . \Omega$ can be a finite or countable set, in which case the stochastic process is called a discrete stochastic process, or an uncountable set such as an interval $\Omega \subset \mathbb{R}$ or even a domain $\Omega \subset \mathbb{R}^{d}$. In the case where $\Omega$ is a spatial domain, the random process is rather called a random field. A stochastic process can be equivalently seen as a measurable function

$$
u:(x, \theta) \in \Omega \times \Theta \mapsto u(x, \theta) \in \mathcal{E},
$$

or still as a random variable with values in a space of functions defined on $\Omega$ with values in $\mathcal{E}$. The equivalence between these different interpretations require some technical considerations $[67,77,61]$. In the following, one restricts the presentation to scalar random processes, i.e. $\mathcal{E}=\mathbb{R}$.

The probabilistic characterization of a stochastic process then requires the probabilistic characterization of a set of random variables, eventually uncountable. In fact, a random process can be completely characterized by its finite dimensional probability laws [1,67], which are the joint probability laws of all finite sets of random variables $\left\{u_{x_{1}}, \ldots, u_{x_{n}}\right\}, n \in \mathbb{N}, x_{i} \in \Omega$.

In the following, we consider the representation and discretization of second order processes (for an introduction to generalized random processes, see $[41,67,30,55]$ ). Various discretization techniques are available in the literature (see $[115,117,83,125,60])$. We here present two techniques classically used in the context of spectral stochastic methods: the Karhunen-Loève decomposition and the polynomial chaos decomposition.

\section{A.2 Karhunen-Loève decomposition}

Karhunen-Loève decomposition [76,58] applies to second order stochastic processes $u \in L^{2}(\Omega) \otimes$ $L^{2}(\Theta, d P)$. It consists in decomposing the random process $u$ under the form:

$$
u(x, \theta)=\mu_{u}(x)+\sum_{i=1}^{\infty} \sqrt{\sigma_{i}} w_{i}(x) \zeta_{i}(\theta)
$$

where $\mu_{u}$ is the mean value of $u$, where functions $w_{i}(x)$ form a particular Hilbertian basis of $L^{2}(\Omega)$, where the $\zeta_{i} \in L^{2}(\Theta, d P)$ are centered uncorrelated random variables with unit variance and where the $\sigma_{i}$ are positive constants. Couples $\left(w_{i}, \sigma_{i}\right) \in L^{2}(\Omega) \times \mathbb{R}^{+}$are solutions of an eigenproblem ${ }^{12}$

$$
\int_{\Omega} C_{u}(x, y) w_{i}(y) d y=\sigma_{i} w_{i}(x)
$$

where $C_{u}$ is the covariance function of $u$, defined by:

$$
C_{u}(x, y)=E\left(\left(u(x, \theta)-\mu_{u}(x)\right)\left(u(y, \theta)-\mu_{u}(y)\right)\right) .
$$

Couples $\left(w_{i}, \sigma_{i}\right)$ are then the eigenpairs of operator

$$
\begin{aligned}
T_{u}: w \in L^{2}(\Omega) \mapsto T_{u}(v) & =\int_{\Omega} C_{u}(\cdot, y) w(y) d y \\
& :=<C_{u}, w>_{L^{2}(\Omega)} .
\end{aligned}
$$

$T_{u}$ is called the covariance operator and $C_{u}$ the covariance kernel. Under regularity assumptions on $C_{u}$ (e.g. $C_{u} \in C(\Omega \times \Omega)$ with $\Omega$ compact, or $C_{u} \in L^{2}(\Omega \times \Omega)$ ), one shows that $T_{u}$ is a continuous self-adjoint positive semi-definite and compact operator from $L^{2}(\Omega)$ to $L^{2}(\Omega)$, so that classical spectral theory applies [105]. $T_{u}$ has a countable set of non-negative eigenvalues with only possible accumulation point zero. The set of its eigenfunctions form a Hilbertian basis

12 Eigenproblem (116) is a homogeneous Fredholm integral equation of second type [32,105]. 
of $L^{2}(\Omega)$. Random process $u$ can then be decomposed under the form (115), where random variables $\zeta_{i}$ are defined by

$$
\begin{aligned}
\zeta_{i}(\theta) & =\frac{1}{\sqrt{\sigma_{i}}}<u-\mu_{u}, w_{i}>_{L^{2}(\Omega)} \\
& =\frac{1}{\sqrt{\sigma_{i}}} \int_{\Omega}\left(u(x, \theta)-\mu_{u}(x)\right) w_{i}(x) d x .
\end{aligned}
$$

The series (115) converges in $L^{2}(\Omega) \otimes L^{2}(\Theta, d P)$ :

$$
\begin{aligned}
& \left\|u-\mu_{u}-\sum_{i=1}^{m} \sqrt{\sigma_{i}} w_{i} \zeta_{i}\right\|_{L^{2}(\Omega) \otimes L^{2}(\Theta, d P)}^{2} \\
& =\left\|u-\mu_{u}\right\|_{L^{2}(\Omega) \otimes L^{2}(\Theta, d P)}^{2}-\sum_{i=1}^{m} \sigma_{i} \underset{m \rightarrow \infty}{\longrightarrow} 0 .
\end{aligned}
$$

In the case of a Gaussian random process, random variables $\zeta_{i}$ are uncorrelated and hence independent Gaussian random variables. The Karhunen-Loève decomposition is then very interesting in this case since it allows representing the process in terms of a set of random variables whose probability law is completely and easily characterized. In the case of a non-Gaussian random process, this decomposition is still valid. However, the probabilistic characterization of the set of random variables is more delicate.

Remark 18 - In very particular cases, eigenproblem (116) admits analytical solutions. In the general case, the resolution asks for ad hoc numerical techniques [4].

The stochastic discretization of the process consists in truncating the Karhunen-Loève up to an order $m$, keeping the $m$ dominant eigenvalues:

$$
u(x, \theta) \approx u_{m}(x, \theta)=\mu_{u}(x)+\sum_{i=1}^{m} \sqrt{\sigma_{i}} w_{i}(x) \zeta_{i}(\theta) .
$$

It results the following optimality property: the truncated Karhunen-Loève decomposition (120) is the optimal decomposition of the process with respect to the natural norm in $L^{2}(\Omega) \otimes$ $L^{2}(\Theta, d P)$ over the set of decompositions of type $\mu_{u}(x)+\sum_{i=1}^{m} w_{i}(x) \nu_{i}(\theta)$, with $w_{i} \in L^{2}(\Omega)$ and $\zeta_{i} \in L^{2}(\Theta, d P)$ :

$$
\begin{aligned}
& \left\|u-u_{m}\right\|_{L^{2}(\Omega) \otimes L^{2}(\Theta, d P)}^{2} \\
& =\min _{\substack{w_{i} \in L^{2}(\Omega) \\
\nu_{i} \in L^{2}(\Theta, d P)}}\left\|u-\mu_{u}-\sum_{i=1}^{m} w_{i} \nu_{i}\right\|_{L^{2}(\Omega) \otimes L^{2}(\Theta, d P)}^{2} .
\end{aligned}
$$

Remark 19 - Let us note that generalizations of Karhunen-Loève expansion exists in the case where the covariance operator $T_{u}$ is not compact or not continuous [33,13].

\section{A.3 Hilbert Karhunen-Loève decomposition}

A natural extension of Karhunen-Loève decomposition, called Hilbert Karhunen-Loève [75, 38], consists in searching an optimal decomposition of the process $u \in \mathcal{V} \otimes L^{2}(\Theta, d P)$, where $\mathcal{V}$ is a Hilbert space of functions defined on $\Omega$. The space $\mathcal{V} \otimes L^{2}(\Theta, d P)$ is endowed with the inner product

$$
<\cdot, \cdot>_{\mathcal{V} \otimes L^{2}(\Theta, d P)}=E\left(<\cdot, \cdot>_{\mathcal{V}}\right),
$$

where $\langle\cdot, \cdot\rangle_{\mathcal{V}}$ is an inner product on $\mathcal{V}$. We introduce the linear operator $T_{u}$ from $\mathcal{V}$ to $\mathcal{V}$ defined by

$$
T_{u}(w)=<C_{u}, w>_{\mathcal{v}}:=E\left(\left(u-\mu_{u}\right)<\left(u-\mu_{u}\right), w>_{\mathcal{v}}\right),
$$


where $C_{u} \in \mathcal{V} \otimes \mathcal{V}$ is the covariance function of random process $u$, defined by equation (117). Under regularity assumptions on $C_{u}, T_{u}$ is a continuous self-adjoint positive semi-definite and compact operator from $\mathcal{V}$ to $\mathcal{V}$ and classical spectral theory applies. Eigenfunctions $w_{i}$ of $T_{u}$ form a Hilbertian basis of $\mathcal{V}$ (i.e. a complete set of functions which are orthonormal with respect to inner product $\left.\langle\cdot, \cdot\rangle_{v}\right)$. Denoting $\left(w_{i}, \sigma_{i}\right)$ the eigenpairs of $T_{u}$, one obtains the following decomposition of the random process:

$$
u(x, \theta)=\mu_{u}(x)+\sum_{i=1}^{\infty} \sqrt{\sigma_{i}} w_{i}(x) \zeta_{i}(\theta)
$$

where the $\zeta_{i} \in L^{2}(\Theta, d P)$ are centered uncorrelated random variables with unit variance. This decomposition is convergent in $\mathcal{V} \otimes L^{2}(\Theta, d P)$ :

$$
\begin{aligned}
& \left\|u-\mu_{u}-\sum_{i=1}^{m} \sqrt{\sigma_{i}} w_{i} \zeta_{i}\right\|_{\mathcal{V} \otimes L^{2}(\Theta, d P)}^{2} \\
& =\left\|u-\mu_{u}\right\|_{\mathcal{V} \otimes L^{2}(\Theta, d P)}^{2}-\sum_{i=1}^{m} \sigma_{i} \underset{m \rightarrow \infty}{\longrightarrow} 0 .
\end{aligned}
$$

One can then obtain a discretized version of the random process by truncating the decomposition:

$$
u(x, \theta) \approx u_{m}(x, \theta)=\mu_{u}(x)+\sum_{i=1}^{m} \sqrt{\sigma_{i}} w_{i}(x) \zeta_{i}(\theta) .
$$

This truncated decomposition verifies the following optimality property: the truncated Hilbert Karhunen-Loève expansion (124) is the optimal decomposition of the random process with respect to the natural norm in $\mathcal{V} \otimes L^{2}(\Theta, d P)$ over the set of decompositions of type $\mu_{u}(x)+$ $\sum_{i=1}^{m} w_{i}(x) \nu_{i}(\theta)$, with $w_{i} \in \mathcal{V}$ and $\nu_{i} \in L^{2}(\Theta, d P)$ :

$$
\begin{aligned}
& \left\|u-u_{m}\right\|_{\mathcal{V} \otimes L^{2}(\Theta, d P)}^{2} \\
& =\min _{\substack{w_{i} \in \mathcal{V} \\
\nu_{i} \in L^{2}(\Theta, d P)}}\left\|u-\mu_{u}-\sum_{i=1}^{m} w_{i} \nu_{i}\right\|_{\mathcal{V} \otimes L^{2}(\Theta, d P)}^{2} .
\end{aligned}
$$

\section{A.4 Polynomial chaos decomposition}

Another type of representation of second order random processes consists in using a polynomial chaos expansion. As suggested by Wiener [130], any random variables in $L^{2}(\Theta, d P)$ can be represented as a series of polynomials in independent standard Gaussian random variables $[23,48,57]$.

Let us denote by $\boldsymbol{\xi}=\left\{\xi_{i}\right\}_{i \in \mathbb{N}^{*}}$ a countable set of independent standard Gaussian random variables. Orthogonal polynomials in $\boldsymbol{\xi}$ are the multidimensional Hermite polynomials. By introducing the set of finite length multi-indices

$$
\mathcal{J}=\left\{\alpha=\left(\alpha_{i}\right)_{i \in \mathbb{N}^{*}} \in \mathbb{N}^{\mathbb{N}^{*}} ;|\alpha|=\sum_{i \in \mathbb{N}^{*}} \alpha_{i}<\infty\right\},
$$

Hermite polynomials can be written as:

$$
H_{\alpha}(\boldsymbol{\xi})=\prod_{i=1}^{\infty} h_{\alpha_{i}}\left(\xi_{i}\right)
$$

where the $h_{n}(x)$ are unidimensional Hermite polynomials which are orthonormal polynomial with respect to Gaussian measure $\varphi(x) d x=\frac{1}{\sqrt{2 \pi}} \exp \left(-\frac{x^{2}}{2}\right) d x$. The homogeneous chaos of degree $p$ is the space $\mathcal{H}_{p}$ defined by:

$$
\mathcal{H}_{p}=\operatorname{span}\left\{H_{\alpha} ; \alpha \in \mathcal{J},|\alpha|=p\right\} .
$$


The polynomial chaos of degree $p$ is then defined by

$$
\oplus_{k=0}^{p} \mathcal{H}_{k}
$$

We have the following orthogonal decomposition of the space of second order random variables:

$$
L^{2}(\Theta, d P)=\overline{\oplus_{k=1}^{\infty} \mathcal{H}_{k}} .
$$

In other terms, the set of polynomials $\left\{H_{\alpha}\right\}_{\alpha \in \mathcal{J}}$ form a Hilbertian basis of $L^{2}(\Theta, d P)$. A stochastic process (or field) $u(x, \theta)$ can then be decomposed as follows:

$$
\begin{aligned}
& u(x, \theta)=\sum_{\alpha \in \mathcal{J}} u_{\alpha}(x) H_{\alpha}(\boldsymbol{\xi}(\theta)), \\
& u_{\alpha}(x)=<u(x, \cdot), H_{\alpha}(\boldsymbol{\xi}(\cdot))>_{L^{2}(\Theta, d P)}=E\left(u(x, \theta) H_{\alpha}(\boldsymbol{\xi}(\theta))\right) .
\end{aligned}
$$

This decomposition converges in $L^{2}(\Theta, d P)$ [26] (and eventually in other $L^{p}$ spaces [57]). This representation can be used as a complement to Karhunen-Loève expansions (115) or (122). Indeed, it allows for a representation of random variables which appear in these expansions: $\zeta_{i}(\theta)=\sum_{\alpha \in \mathcal{J}} \zeta_{i, \alpha} H_{\alpha}(\boldsymbol{\xi}(\theta))$. An approximation of the random process is then obtained by truncating the polynomial chaos to a finite degree, with a finite number of random variables.

\section{B Generalized spectral decomposition for linear elliptic symmetric problems}

Here, we detail the concept of generalized spectral decomposition for the case of linear elliptic symmetric problems, introduced in [89]. In this case, the method can be interpreted as a natural extension of the Hilbert Karhunen-Loève decomposition. Let us note that the following results apply to more general symmetric elliptic problems formulated in tensor product spaces (i.e. not only to stochastic PDEs).

Let $u \in \mathcal{V} \otimes \mathcal{S}$ denote the solution of problem

$$
A(u, v)=B(v) \quad \forall v \in \mathcal{V} \otimes \mathcal{S},
$$

where $A$ is a linear continuous coercive symmetric bilinear form on the Hilbert space $\mathcal{V} \otimes \mathcal{S}$ and where $B$ is a linear continuous form on $\mathcal{V} \otimes \mathcal{S}$. Bilinear form $A$ defines a norm and an inner product on Hilbert space $\mathcal{V} \otimes \mathcal{S}$, respectively defined by: $\forall u, v \in \mathcal{V} \otimes \mathcal{S}$,

$$
\|u\|_{A}=A(u, u), \quad\left\langle u, v>_{A}=A(u, v) .\right.
$$

A natural extension of classical spectral decomposition consists in using the norm $\|\cdot\|_{A}$ in the optimality condition (72), i.e. by defining an optimal decomposition $u_{M}=\sum_{i=1}^{M} w_{i} \lambda_{i}$, with $w_{i} \in \mathcal{V}$ and $\lambda_{i} \in \mathcal{S}$, as follows:

$$
\left\|u-u_{M}\right\|_{A}^{2}=\min _{\substack{\left\{\lambda_{i}\right\}_{i=1}^{M} \in(\mathcal{S})^{M} \\\left\{w_{i}\right\}_{i=1}^{M} \in(\mathcal{V})^{M}}}\left\|u-\sum_{i=1}^{M} w_{i} \lambda_{i}\right\|_{A}^{2}
$$

\section{B.1 Generalized spectral decomposition}

\section{B.1.1 Optimal order 1 decomposition}

Let us first consider the case where $M=1$ and let us denote by $(\lambda, w) \in \mathcal{S} \times \mathcal{V}$ the optimal couple verifying:

$$
\|u-\lambda w\|_{A}^{2}=\min _{\substack{\lambda \in \mathcal{S} \\ w \in \mathcal{V}}}\|u-\lambda w\|_{A}^{2}
$$


Stationarity conditions of optimization problem (127) with respect to $w$ and $\lambda$ respectively write:

$$
\begin{array}{ll}
A\left(w^{*} \lambda, w \lambda\right)=B\left(w^{*} \lambda\right) & \forall w^{*} \in \mathcal{V} \\
A\left(w \lambda^{*}, w \lambda\right)=B\left(w \lambda^{*}\right) & \forall \lambda^{*} \in \mathcal{S} .
\end{array}
$$

Let us introduce the mapping $F: \mathcal{S} \rightarrow \mathcal{V}$ where $w=F(\lambda)$ is the solution of equation (128) for a given $\lambda$. Let us also introduce the mapping $f: \mathcal{V} \rightarrow \mathcal{S}$ where $\lambda=f(w)$ is the solution of equation (129) for a given $w$. The simultaneous verification of (128) and (129) imposes the following relations:

$$
w=F(\lambda) \quad \text { and } \quad \lambda=f(w) .
$$

Equations (130) can be interpreted as a problem on $w$ :

$$
T(w)=w, \quad \text { with } \quad T(w)=F \circ f(w) .
$$

If $w$ solves problem (131), it satisfies

$$
\|u-f(w) w\|_{A}^{2}=\|u\|_{A}^{2}-\sigma(w),
$$

with

$$
\sigma(w)=A(f(w) w, f(w) w) \equiv\|f(w) w\|_{A}^{2} .
$$

The optimal function $w$ is then the fixed point of $T$ that maximizes $\sigma(w)$. In fact, operator $T: \mathcal{V} \rightarrow \mathcal{V}$ is homogeneous of degree 1 and $\sigma: \mathcal{V} \rightarrow \mathbb{R}^{+}$is homogeneous of degree 0, i.e. $\forall \alpha \in \mathbb{R} \backslash\{0\}, T(\alpha w)=\alpha T(w)$ and $\sigma(\alpha w)=\sigma(w)$. Problem (131) can then be interpreted as a generalized eigenproblem with associated generalized Rayleigh quotient $\sigma(w)$. The optimal function $w$ can then be interpreted as the dominant eigenfunction of $T$, associated with generalized eigenvalue $\sigma(w)$. This generalized eigenproblem (131) can be written in a more conventional way by introducing a "rescaled" operator $\widetilde{T}(w)=\sigma(w) T(w)$. Problem (131) is then equivalent to:

$$
\widetilde{T}(w)=\sigma(w) w
$$

Remark 20 - Equations (130) can be equivalently interpreted as a problem on $\lambda$ :

$$
T^{\diamond}(\lambda)=\lambda, \quad \text { with } \quad T^{\diamond}(\lambda)=f \circ F(\lambda),
$$

which can be rewritten as follows:

$$
\widetilde{T}^{\diamond}(\lambda)=\sigma^{\diamond}(\lambda) \lambda,
$$

where $\widetilde{T}^{\diamond}(\lambda)=\sigma^{\diamond}(\lambda) T^{\diamond}(\lambda)$ and

$$
\sigma^{\diamond}(\lambda)=A(\lambda F(\lambda), \lambda F(\lambda))=\|\lambda F(\lambda)\|_{A}^{2} .
$$

If $\lambda$ solves (135), then

$$
\|u-F(\lambda) \lambda\|_{A}^{2}=\|u\|_{A}^{2}-\sigma^{\diamond}(\lambda),
$$

and if we let $w=F(\lambda)$ be the associated deterministic function, then $\lambda F(\lambda)=w f(w), w$ solves eigenproblem $(131)$ and $\sigma^{\diamond}(\lambda)=\sigma(w)$. 


\section{B.1.2 Optimal order $M$ decomposition}

Now, let us consider the case of an order $M$ decomposition. We use the same notations as in section 6.1 , where $\Lambda=\left(\lambda_{i}\right)_{i=1}^{M} \in(\mathcal{S})^{M}, W=\left(w_{i}\right)_{i=1}^{M} \in(\mathcal{V})^{M}$. The order $M$ decomposition is denoted by $u_{M}:=W \cdot \Lambda$. The optimal decomposition of order $M$ can be naturally defined as the one which minimizes $\left\|u-u_{M}\right\|_{A}$. Stationarity conditions with respect to deterministic functions and stochastic functions respectively lead to equations (86) and (87). Following section 6.1 , we introduce the equivalent problem on $W$ :

$$
T(W)=W, \quad \text { with } \quad T=F \circ f .
$$

If $W$ solves problem (138), it satisfies

$$
\|u-W \cdot f(W)\|_{A}^{2}=\|u\|_{A}^{2}-\sigma(W),
$$

with

$$
\sigma(W)=A(W \cdot f(W), W \cdot f(W)) \equiv\|W \cdot f(W)\|_{A}^{2}
$$

$\sigma(W)$ can be interpreted as a Rayleigh quotient ${ }^{13}$. The optimal reduced basis $W$ is such that it maximizes $\sigma(W)$. The subspace spanned by an optimal $W$ is called the dominant eigenspace. In fact, we classically show that if $W$ and $W^{*}$ span the same subspace, then ${ }^{14} \sigma(W)=\sigma\left(W^{*}\right)$, which means that $\sigma$ can be equivalently interpreted as a real-valued function on $M$-dimensional linear subspaces of $\mathcal{V}$.

The generalized spectral decomposition $u_{M}=W \Lambda$, with $\Lambda=f(W)$, is the optimal decomposition of order $M$ with respect to the norm $\|\cdot\|_{A}$. Since problem (138) is not a classical eigenproblem, functions $w_{i}$ and $\lambda_{i}$ do not verify classical simultaneous orthogonality properties (see below for classical spectral decompositions).

Remark 21 - Connection between generalized eigenfunctions and generalized eigenspaces. Of course, for classical eigenproblems, the $k$ dominant generalized eigenfunctions span the $k$-dimensional dominant eigenspace. For the above eigen-like problem, this property is not necessarily true. In particular, the dominant eigenfunction of $T$ is not necessarily included in the $k$-dimensional dominant eigenspace of $T$. That means that for obtaining the optimal order $M$ decomposition, one has to consider the research of the $M$-dimensional dominant generalized eigenspace and not the research of generalized eigenfunctions independently. However, in practice, the above mentioned property is almost verified (can be observed by computing angles between generated linear subspaces).

\section{B.1.3 Sub-optimal Order $M$ decomposition}

In the case where we define the couples $\left(w_{i}, \lambda_{i}\right)$ of the decomposition one after the other, i.e. if we define $\left(w_{i}, \lambda_{i}\right)$ such that it minimizes $\left\|u-u_{i-1}-\lambda_{i} w_{i}\right\|$, we can write an eigen-like problem on a deflated operator $T\left(\cdot ; u_{i-1}\right)$ (see section 6.2.2) and define an associated generalized Rayleigh quotient $\sigma\left(w ; u_{i-1}\right)$. With this one-by-one construction, the optimal decomposition verifies

$$
\left\|u-u_{M}\right\|_{A}^{2}=\|u\|_{A}^{2}-\sum_{i=1}^{M} \sigma\left(w_{i} ; u_{i-1}\right)
$$

which leads to an error greater than (139), obtained with the dominant eigenspace of the initial operator. This fact, connected to remark 21, explains why in GSD algorithms, a global update of random variables (or deterministic functions) with respect to the initial problem (not deflated) generally improves the obtained decomposition.

13 One can also introduce an associated generalized matrix Rayleigh quotient $\Sigma(W)[114]$, defined by $(\Sigma(W))_{i j}=A\left(W \cdot f(W), w_{i} f_{j}(W)\right)$, and such $\sigma(W)=\operatorname{Trace}(\Sigma(W))($ see $[89,91])$.

14 This equivalence between elements $W$ spanning the same subspace is due the homogeneity property of $\sigma$, i.e. $\sigma(W \cdot P)=\sigma(W)$ for all invertible matrix $P$, where $(W \cdot P)_{i}:=\sum_{j=1}^{M} w_{j} P_{j i}$. 
B.2 Connection with classical spectral decompositions

In the case where inner product $A(\cdot, \cdot)$ results from a tensorization of inner products on $\mathcal{V}$ and $\mathcal{S}$, i.e. if we can write

$$
A\left(\lambda w, \lambda w^{*}\right)=<w, w^{*}>_{\mathcal{V}}<\lambda, \lambda>_{\mathcal{S}}
$$

mappings $F$ and $f$ in equation (130) write as follows:

$$
\begin{aligned}
& F(\lambda)=<\lambda, \lambda>_{\mathcal{S}}^{-1}<u, \lambda>_{\mathcal{S}}, \\
& f(w)=<w, w>_{\mathcal{v}}^{-1}<u, w>_{\mathcal{v}} .
\end{aligned}
$$

Operator $\widetilde{T}$ and function $\sigma(w)$ then write:

$$
\begin{aligned}
& \widetilde{T}(w)=<u,<u, w>_{\mathcal{V}}>_{\mathcal{S}}, \\
& \sigma(w)=<<w, u>_{\mathcal{V}},<u, w>_{\mathcal{V}}>_{\mathcal{S}}=<w, \widetilde{T}(w)>_{\mathcal{v}} .
\end{aligned}
$$

$\widetilde{T}$ appears as a classical correlation operator associated with particular metrics on $\mathcal{V}$ and $\mathcal{S}$. In this case, problem (134) is then a classical eigenproblem. Eigenfunctions $w_{i}$ are orthogonal with respect to inner product $\langle\cdot, \cdot\rangle_{\nu}$ and associated functions $\lambda_{i}=f\left(w_{i}\right)$ are orthogonal with respect to inner product $\langle\cdot, \cdot\rangle_{\S}$. Retaining the $M$ dominant eigenfunctions then leads to a Hilbert Karhunen-Loève decomposition

$$
u_{M}=\sum_{i=1}^{M} w_{i} f\left(w_{i}\right) \equiv \sum_{i=1}^{M} \sqrt{\sigma\left(w_{i}\right)} \frac{1}{\left\|w_{i} \lambda_{i}\right\|_{A}} w_{i} \lambda_{i}
$$

verifying

$$
\left\|u-u_{M}\right\|_{A}^{2}=\|u\|_{A}^{2}-\sum_{i=1}^{M} \sigma\left(w_{i}\right) .
$$

In this case, we can show that sub-optimal spectral decomposition defined in section B.1.3 coincides with the optimal spectral decomposition of order $M$.

Example 2 (Deterministic operator)

If $A(u, v)=E(a(u, v))$, where $a$ is a deterministic continuous coercive symmetric bilinear form on $\mathcal{V}$, property (141) is then verified with $\left\langle w^{*}, w>_{\mathcal{V}}=a\left(w^{*}, w\right)\right.$ and $\left\langle\lambda^{*}, \lambda>_{\mathcal{S}}=\right.$ $E\left(\lambda \lambda^{*}\right)$, which is the classical inner product in $L^{2}\left(\boldsymbol{\Xi}, d P_{\boldsymbol{\xi}}\right)$. The generalized spectral decomposition then exactly coincides with the classical Hilbert Karhunen-Loève decomposition of $u \in \mathcal{V} \otimes L^{2}\left(\boldsymbol{\Xi}, d P_{\boldsymbol{\xi}}\right)$, where the inner product on $\mathcal{V}$ is based on the bilinear form $a$. This case is encountered for stochastic elliptic symmetric PDE with deterministic operator and stochastic right-hand side.

Example 3 (Operator with order 1 decomposition)

If $A(u, v)=E(\alpha(\boldsymbol{\xi}) a(u, v))$, where $a$ is a deterministic continuous coercive symmetric bilinear form on $\mathcal{V}$ and $\alpha(\boldsymbol{\xi})$ is a random variable such that $0<\alpha_{0} \leqslant \alpha(\boldsymbol{\xi}) \leqslant \alpha_{1}<\infty$ almost surely, property (141) follows with $\left\langle w, w^{*}>_{\mathcal{V}}=a\left(w, w^{*}\right)\right.$ and $\left\langle\lambda, \lambda^{*}>_{\mathcal{S}}=E\left(\alpha \lambda \lambda^{*}\right)\right.$. This case is encountered for stochastic linear elliptic symmetric PDE where the operator is the product of a random variable by a deterministic operator (i.e. when the operator admits an "order 1 spectral decomposition").

\section{References}

1. R. J. Adler. The Geometry of Random Fields. John Wiley \& Sons, Chichester, 1981.

2. A. Ammar, B. Mokdad, F. Chinesta, and R. Keunings. A new family of solvers for some classes of multidimensional partial differential equations encountered in kinetic theory modelling of complex fluids. Journal of Non-Newtonian Fluid Mechanics, 139(3):153$176,2006$. 
3. A. Ammar, B. Mokdad, F. Chinesta, and R. Keunings. A new family of solvers for some classes of multidimensional partial differential equations encountered in kinetic theory modelling of complex fluids part II: Transient simulation using space-time separated representations. Journal of Non-Newtonian Fluid Mechanics, 144(2-3):98-121, 2007.

4. K. E. Atkinson. The Numerical Solution of Integral Equations of the Second Kind. Cambridge University Press, Cambridge, 1997.

5. I. Babuška and P. Chatzipantelidis. On solving elliptic stochastic partial differential equations. Computer Methods in Applied Mechanics and Engineering, 191:4093-4122, 2002 .

6. I. Babuška and J. Chleboun. Effects of uncertainties in the domain on the solution of neumann boundary value problems in two spatial dimensions. Mathematics of Computation, 71(240):1339-1370, 2002.

7. I. Babuška, K.-M. Liu, and R. Tempone. Solving stochastic partial differential equations based on the experimental data. TICAM Report, 02-18, 2002.

8. I. Babuška, F. Nobile, and R. Tempone. A stochastic collocation method for elliptic partial differential equations with random input data. SIAM J. Num. Anal., 45(3):10051034, 2007.

9. I. Babuška, R. Tempone, and G. E. Zouraris. Solving elliptic boundary value problems with uncertain coefficients by the finite element method: the stochastic formulation. Computer Methods in Applied Mechanics and Engineering, 194:1251-1294, 2005.

10. I. Babuška, R. Tempone, and G.E. Zouraris. Galerkin finite element approximations of stochastic elliptic differential equations. TICAM Report, 02-38, 2002.

11. M. Barrault, Y. Maday, N. C. Nguyen, and A. T. Patera. An empirical interpolation method: application to efficient reduced-basis discretization of partial differential equations. Comptes Rendus Mathematique, 339(9):667-672, 2002.

12. F. E. Benth and J. Gjerde. Convergence rates for finite element approximations of stochastic partial differential equations. Stochastics and Stochastics Rep., 63(3-4):313-326, 1998.

13. A. Berlinet and C. Thomas-Agnan. Reproducing Kernel Hilbert Spaces in Probability and Statistics. Kluwer, Dordrecht, 2004.

14. M. Berveiller. Stochastic finite elements: intrusive and non-intrusive methods for reliability analysis. PhD thesis, Université Blaise Pascal, Clermont-Ferrand, 2005.

15. M. Berveiller, B. Sudret, and M. Lemaire. Stochastic finite element: a non intrusive approach by regression. European Journal of Computational Mechanics, 15:81-92, 2006.

16. P. Besold. Solutions to Stochastic Partial Differential Equations as Elements of Tensor Product Spaces. PhD thesis, Georg-August-Universität, Göttingen, 2000.

17. G. Blatman and B. Sudret. Sparse polynomial chaos expansions and adaptive stochastic finite elements using a regression approach. Comptes Rendus Mécanique, 336(6):518-523, 2007.

18. G. Blatman, B. Sudret, and M. Berveiller. Quasi random numbers in stochastic finite element analysis. Mécanique ES Industries, 8:289-297, 2007.

19. H. Brézis. Analyse fonctionnelle : théorie et applications. Masson, Paris, 1983.

20. C. Le Bris, T. Lelievre, and Y. Maday. Results and questions on a nonlinear approximation approach for solving high-dimensional partial differential equations, e-print arXiv:0811.0474v1, 2008.

21. H-J. Bungartz and M. Griebel. Sparse grids. Acta. Numer., 13:147-269, 2004.

22. R. E. Caflisch. Monte carlo and quasi-monte carlo methods. Acta. Numer., 7:1-49, 1998.

23. R.H. Cameron and W.T. Martin. The orthogonal development of non-linear functionals in series of fourier-hermite functionals. The Annals of Mathematics, Second Series, 48(2):385-392, 1947.

24. C. Canuto, M.Y. Hussaini, A. Quateroni, and T.A. Zang. Spectral methods in fluid dynamics. Springer-Verlag, 1988.

25. C. Canuto and T. Kozubek. A fictitious domain approach to the numerical solution of pdes in stochastic domains. Numerische Mathematik, 107(2):257-293, 2007.

26. Y. Cao. On the rate of convergence of wiener-ito expansion for generalized random variables. Stochastics, 78:179-187, 2006.

27. F. Chinesta, A. Ammar, F. Lemarchand, P. Beauchene, and F. Boust. Alleviating mesh constraints: Model reduction, parallel time integration and high resolution homogenization. Computer Methods in Applied Mechanics and Engineering, 197(5):400-413, 2008.

28. S. Choi, R.V. Grandhi, and R. A. Canfield. Structural reliability under non-gaussian stochastic behavior. Computers and Structures, 82:1113-1121, 2004. 
29. S. Choi, R.V. Grandhi, R. A. Canfield, and C.L. Pettit. Polynomial chaos expansion with latin hypercube sampling for estimating response variability. AIA A Journal, 42(6):11911198, 2004.

30. G. Christakos. Random Field Models in Earth Sciences. Academic Press, San Diego, CA, 1992.

31. P. G. Ciarlet. The Finite Element Method for Elliptic Problems. North-Holland, Amsterdam, 1978.

32. R. Courant and D. Hilbert. Methods of Mathematical Physics. John Wiley \& Sons, Chichester, 1989.

33. R. Dautray and J.-L. Lions. Mathematical Analysis and Numerical Methods for Science and Technology, Vol. 3, Spectral theory and applications. Springer-Verlag, Berlin, 1990.

34. M. Deb, I. Babuška, and J. T. Oden. Solution of stochastic partial differential equations using galerkin finite element techniques. Computer Methods in Applied Mechanics and Engineering, 190:6359-6372, 2001.

35. J. E. Dennis and R. B. Schnabel. Numerical Methods for Unconstrained Optimization and Nonlinear Equations. SIAM, 1996.

36. O. Ditlevsen and H. Madsen. Strutural Reliability Methods. J. Wiley and Sons, Chichester, 1996.

37. J. L. Doob. Stochastic Processes. John Wiley \& Sons, Chichester, 1953.

38. A. Doostan, R. Ghanem, and J. Red-Horse. Stochastic model reductions for chaos representations. Computer Methods in Applied Mechanics and Engineering, 196(37-40):39513966, 2007.

39. C.G. Webster F. Nobile, R. Tempone. A sparse grid stochastic collocation method for partial differential equations with random input data. SIAM Journal on Numerical Analysis, 46(5):2309-2345, 2007.

40. P. Frauenfelder, C. Schwab, and R. A. Todor. Finite elements for elliptic problems with stochastic coefficients. Computer Methods in Applied Mechanics and Engineering, 194(25):205-228, 2005.

41. I. M. Gel'fand and N. Y. Vilenkin. Generalized Functions - Volume 4: Applications of harmonic analysis. Academic Press, New York, NY, 1964.

42. T. Gerstner and M. Griebel. Numerical integration using sparse grids. Numer. Algorithms, 18:209-232, 1998.

43. T. Gerstner and M. Griebel. Dimension-adaptive tensor-product quadrature. Computing, $71(1): 65-87,2003$.

44. R. Ghanem. Ingredients for a general purpose stochastic finite elements implementation. Computer Methods in Applied Mechanics and Engineering, 168:19-34, 1999.

45. R. Ghanem. Stochastic finite elements for heterogeneous media with multiple random non-gaussian properties. ASCE J. Engrg. Mech., 125:24-40, 1999.

46. R. Ghanem and R. M. Kruger. Numerical solution of spectral stochastic finite element systems. Computer Methods in Applied Mechanics and Engineering, 129:289-303, 1996.

47. R. Ghanem, G. Saad, and A. Doostan. Efficient solution of stochastic systems: application to the embankment dam problem. Structural Safety, 29(3):238-251, 2007.

48. R. Ghanem and P. Spanos. Stochastic finite elements: a spectral approach. Springer, Berlin, 1991.

49. D. Ghiocel and R. Ghanem. Stochastic finite-element analysis of seismic soil-structure interaction. ASCE Journal Engrg. Mech., 128(1):66-77, 2002.

50. G. H. Golub and C. F. Van Loan. Matrix Computations, 3rd ed. Johns Hopkins University Press, Baltimore, MD, 1996.

51. P. Gosselet and C. Rey. On a selective reuse of Krylov subspaces in Newton-Krylov approaches for nonlinear elasticity. In Domain decomposition methods in science and engineering, pages 419-426, 2002.

52. M. Grigoriu. Applied non-Gaussian Processes. Prentice Hall, Englewood Cliffs, NJ, 1995.

53. M. Grigoriu. Stochastic Calculus - Applications in Science and Engineering. Birkhäuser Verlag, Basel, 2002.

54. M.A. Gutiérrez and S. Krenk. Stochastic finite element methods. In E. Stein et al., editor, Encyclopedia of Computational Mechanics, volume 2:Solids and Structures, pages 657-681. Wiley, Chichester, 2006.

55. H. Holden, B. Øksendal, J. Ubøe, and T. Zhang. Stochastic Partial Differential Equations. Birkhäuser, 1996.

56. Debusschere B. J., Najm H. N., Pebray P. P., Knio O. M., Ghanem R. G., and Le Maitre O. P. 
57. S. Janson. Gaussian Hilbert Spaces. Cambridge University Press, Cambridge, 1997.

58. K. Karhunen. Zur spektraltheorie stochastischer prozesse. Ann. Acad. Sci. Fenn., 34, 1946.

59. A. Keese. Numerical Solution of Systems with Stochastic Uncertainties - A General Purpose Framework for Stochastic Finite Elements. PhD thesis, Technische Universität Braunschweig, Brunswick, 2003.

60. A. Keese. A review of recent developments in the numerical solution of stochastic pdes (stochastic finite elements). Technical Report 2003-6, Technical report, Institute of Scientific Computing, Tech. Univ. Braunschweig, Germany, 2003. http://opus.tubs.de/opus/volltexte/2003/504/.

61. A. Keese and H. G. Mathhies. Numerical methods and Smolyak quadrature for nonlinear stochastic partial differential equations. SIAM J. Sci. Comput., 83, 2003.

62. A. Keese and H. G. Mathhies. Adaptivity and sensitivity for stochastic problems. In P. D. Spanos and G. Deodatis, editors, Computational Stochastic Mechanics, volume 4, pages 311-316. Millpress, Rotterdam, 2004.

63. A. Keese and H. G. Mathhies. Hierarchical parallelisation for the solution of stochastic finite element equations. Computer Methods in Applied Mechanics and Engineering, $83: 1033-1047,2005$.

64. A. Khuri and J. Cornell. Response Surfaces: Designs and Analyses. Marcel Dekker, New York, NY, 1987.

65. M. Kleiber and T.D. Hien. The Stochastic Finite Element Method. Basic Perturbation Technique and Computer Implementation. John Wiley \& sons, Chichester, 1992.

66. P. E. Kloeden and E. Platen. Numerical Solution of Stochastic Differential Equations. Springer, Berlin, 1995.

67. P. Krée and C. Soize. Mathematics of Random Phenomena. D. Reidel, Dordrecht, 1986.

68. P. Ladevèze. Nonlinear Computational Structural Mechanics - New Approaches and Non-Incremental Methods of Calculation. Springer Verlag, 1999.

69. P. Ladevèze and E. Florentin. Verification of stochastic models in uncertain environments using the constitutive relation error method. Computer Methods in Applied Mechanics and Engineering, 196(1-3):225-234, 2006.

70. P. Ladevèze and A. Nouy. On a multiscale computational strategy with time and space homogenization for structural mechanics. Computer Methods in Applied Mechanics and Engineering, 192:3061-3087, 2003.

71. O. P. Le Maître, O. M. Knio, H. N. Najm, and R. G. Ghanem. Uncertainty propagation using Wiener-Haar expansions. Journal of Computational Physics, 197(1):28-57, 2004.

72. O. P. Le Maître, H. N. Najm, R. G. Ghanem, and O. M. Knio. Multi-resolution analysis of wiener-type uncertainty propagation schemes. Journal of Computational Physics, 197(2):502-531, 2004.

73. O.P. Le Maître, O.M. Knio, H.N. Najm, and R. Ghanem. A stochastic projection method for fluid flow. i. basic formulation. J. Comput. Physics, 173:481-511, 2001.

74. O.P. Le Maître, M.T. Reagan, H.N. Najm, R.G. Ghanem, and O.M. Knio. A stochastic projection method for fluid flow. ii. random process. J. Comput. Physics, 181:9-44, 2002.

75. A. Levy and J. Rubinstein. Some properties of smoothed principal component analysis for functional data. Journal of The Optical Society of America, 16(1):28-35, 1999.

76. M. Loève. Fonctions aléatoires du second ordre. CR Acad. Sci. Paris, 220, 1945.

77. M. Loève. Probability Theory. I, fourth edition, in: Graduate Texts in Mathematics, vol. 45. Springer-Verlag, New York, 1977.

78. M. Loève. Probability Theory. II, fourth edition, in: Graduate Texts in Mathematics, vol. 46. Springer-Verlag, New York, 1978.

79. L. Machiels, Y. Maday, and A. T. Patera. Output bounds for reduced-order approximations of elliptic partial differential equations. Computer Methods in Applied Mechanics and Engineering, 190(26-27):3413-3426, 2001.

80. Y. Maday, A. T. Patera, and G. Turinici. Global a priori convergence theory for reducedbasis approximation of single-parameter symmetric coercive elliptic partial differential equations. Comptes Rendus Mathematique, 335(3):289-294, 2002.

81. L. Mathelin and O. Le Maître. Dual-based a posteriori error estimate for stochastic finite element methods. Communications in Applied Mathematics and Computational Science, $2: 83-116,2007$.

82. H. G. Matthies. Uncertainty quantification with stochastic finite elements. In E. Stein et al., editor, Encyclopedia of Computational Mechanics, volume 1, Chapter 27. Wiley, Chichester, 2007. 
83. H. G. Matthies, C. E. Brenner, C. G. Bucher, and C. G. Soares. Uncertainties in probabilistic numerical analysis of structures and solids - stochastic finite elements. Structural Safety, 19(3):283-336, 1997.

84. H. G. Matthies and A. Keese. Galerkin methods for linear and nonlinear elliptic stochastic partial differential equations. Computer Methods in Applied Mechanics and Engineering, 194(12-16):1295-1331, 2005.

85. R.-E. Melchers. Structural reliability analysis and prediction. John Wiley and Sons, 1999.

86. P. B. Nair. On the theoretical foundations of stochastic reduced basis methods. AIAA Paper, 2001-1677, 2001.

87. P. B. Nair and A. J. Keane. Stochastic reduced basis methods. AIAA Journal, 40(8):16531664, 2002.

88. H. Niederreiter. Random Number Generation and quasi-Monte Carlo Methods. SIAM, Philadelphia, PA, 1992.

89. A. Nouy. A generalized spectral decomposition technique to solve a class of linear stochastic partial differential equations. Computer Methods in Applied Mechanics and Engineering, 196(45-48):4521-4537, 2007.

90. A. Nouy. Méthode de construction de bases spectrales généralisées pour l'approximation de problèmes stochastiques. Mécanique E Industries, 8(3):283-288, 2007.

91. A. Nouy. Generalized spectral decomposition method for solving stochastic finite element equations: invariant subspace problem and dedicated algorithms. Computer Methods in Applied Mechanics and Engineering, 197:4718-4736, 2008.

92. A. Nouy, A. Clément, F. Schoefs, and N. Moës. An extended stochastic finite element method for solving stochastic partial differential equations on random domains. Computer Methods in Applied Mechanics and Engineering, 197:4663-4682, 2008.

93. A. Nouy and P. Ladevèze. Multiscale computational strategy with time and space homogenization: a radial-type approximation technique for solving micro problems. International Journal for Multiscale Coputational Engineering, 170(2):557-574, 2004.

94. A. Nouy and O. Le Maître. Generalized spectral decomposition method for stochastic non linear problems. Journal of Computational Physics, 228(1):202-235, 2009.

95. A. Nouy, F. Schoefs, and N. Moës. X-SFEM, a computational technique based on X-FEM to deal with random shapes. European Journal of Computational Mechanics, 16(2):277293, 2007.

96. E. Novak and K. Ritter. Simple cubature formulas with high polynomial exactness. Constr. Approx., 15:499-522, 1999.

97. B. Øksendal. Stochastic Differential Equations. An Introduction with Applications, fifth ed. Springer-Verlag, 1998.

98. A. Papoulis. Probability, Random Variables, and Stochastic Processes. McGraw-Hill, New York, NY, 1984.

99. M. F. Pellissetti and R. G. Ghanem. Iterative solution of systems of linear equations arising in the context of stochastic finite elements. Advances in Engineering Software, 31:607-616, 2000 .

100. K. Petras. Smolyak cubature of given polynomial degree with few nodes for increasing dimension. Numer. Math., 93:729-753, 2003.

101. C.E. Powell and H.C. Elman. Block-diagonal precondioning for the spectral stochastic finite elements systems. Technical Report TR-4879, University of Maryland, Dept. of Computer Science, 2007.

102. W. H. Press, S. A. Teukolsky, W. T. Vetterling, and B. P. Flannery. Numerical Recipes in $C$ - The Art of Scientific Computing. Cambridge University Press, Cambridge, 2nd edition, 1997.

103. B. Puig, F. Poirion, and C. Soize. Non-gaussian simulation using hermite polynomial expansion: convergences. Probabilistic Engineering Mechanics, 17:253-264, 2002.

104. M.T. Reagan, H.N. Najm, R.G. Ghanem, and O.M. Knio. Uncertainty quantification in reacting flow simulations through non-intrusive spectral projection. Combustion and Flames, 132:545-555, 2003.

105. F. Riesz and B. Sz.-Nagy. Functional Analysis. Dover Publications, New York, NY, 1990.

106. F. Risler and C. Rey. Iterative accelerating algorithms with krylov subspaces for the solution to large-scale nonlinear problems. Numerical Algorithms, 23:1-30, 2000.

107. Y. A. Rozanov. Random Fields and Stochastic Partial Differential Equations. Kluwer, Dordrecht, 1998. 
108. Y. Maday N. C. Nguyen A. T. Patera S. Boyaval, C. Le Bris. A reduced basis approach for variational problems with stochastic parameters: Application to heat conduction with variable robin coefficient. Technical Report Rapport de recherche RR-6617 (2008), INRIA, 2008.

109. Y. Saad. Numerical methods for large eigenvalue problems. Halstead Press, New York, 1992.

110. Y. Saad. Analysis of augmented krylov subspace methods. SIAM J. Matrix Anal. Appl., 18(2):435-449, 1997.

111. Y. Saad. Iterative methods for sparse linear systems. PWS Publishing Company, 3rd edition, 2000.

112. S. K. Sachdeva, P. B. Nair, and A. J. Keane. Comparative study of projection schemes for stochastic finite element analysis. Computer Methods in Applied Mechanics and Engineering, 195(19-22):2371-2392, 2006.

113. S. K. Sachdeva, P. B. Nair, and A. J. Keane. Hybridization of stochastic reduced basis methods with polynomial chaos expansions. Probabilistic Engineering Mechanics, $21(2): 182-192,2006$.

114. A. Sameh and Z. Tong. The trace minimization method for the symmetric generalized eigenvalue problem. J. Comput. Appl. Math., 123:155-175, 2000.

115. G. I. Schüeller. A state-of-the-art report on computational stochastic mechanics. Prob. Engrg. Mech., 14:197-321, 1997.

116. G. I. Schüeller and P. D. Spanos (eds). Monte Carlo Simulation. Balkema, Rotterdam, 2001.

117. M. Shinozuka and G. Deodatis. Simulation of stochastic processes and fields. Prob. Engrg. Mech., 14:203-207, 1997.

118. S. A. Smolyak. Quadrature and interpolation formulas for tensor products of certain classes of functions. Sov. Math. Dokl, 3:240-243, 1963.

119. I.M. Sobol. On quasi-monte carlo integrations. Math. Comput. Simulat., 47:103-112, 1998.

120. C. Soize. Non-gaussian positive-definite matrix-valued random fields for elliptic stochastic partial differential operators. Computer Methods in Applied Mechanics and Engineering, $195(1-3): 26-64,2006$

121. C. Soize and R. Ghanem. Physical systems with random uncertainties: chaos representations with arbitrary probability measure. SIAM J. Sci. Comput., 26(2):395-410, 2004.

122. G. Stefanou, A. Nouy, and A. Clément. Identification of random shapes from images through polynomial chaos expansion of random level-set functions. Int. J. for Numerical Methods in Engineering, 2009. doi:10.1002/nme.2546.

123. G. Strang and G. J. Fix. An Analysis of the Finite Element Method. Wellesley-Cambridge Press, Wellesley, MA, 1986.

124. B. Sudret. Global sensitivity analysis using polynomial chaos expansions. Reliability Engineering \& System Safety, 93(7):964-979, 2008.

125. B. Sudret and A. Der Kiureghian. Stochastic finite element methods and reliability. a state-of-the-art report. Technical Report UCB/SEMM-2000/08, Technical Report, Department of Civil \& Environmental Engineering, University of California, Berkeley, CA, 2000 .

126. E. Vanmarcke. Random Fields: Analysis and Synthesis. The MIT Press, Cambridge, MA, 1988.

127. J. B. Walsh. An introduction to stochastic partial differential equations. In Ecole dŠété de Probabilités de Saint Flour XIV. Springer, Berlin, 1984.

128. X. Wan and G.E. Karniadakis. An adaptive multi-element generalized polynomial chaos method for stochastic diffential equations. J. Comp. Phys., 209:617-642, 2005.

129. X. Wan and G.E. Karniadakis. Multi-element generalized polynomial chaos for arbitrary propability measures. SIAM J. Sci. Comp., 28(3):901-928, 2006.

130. N. Wiener. The homogeneous chaos. Am. J. Math., 60:897-936, 1938.

131. K. Willcox and J. Peraire. Balanced model reduction via the proper orthogonal decomposition. AIAA Journal, 40(11):2323-2330, 2002.

132. D. B. Xiu and G. E. Karniadakis. The Wiener-Askey polynomial chaos for stochastic differential equations. SIAM J. Sci. Comput., 24(2):619-644, 2002. 